\title{
Idealized dry quasi-2D mesoscale simulations of cold-air outbreaks over the marginal sea-ice zone with fine and coarse resolution
}

Dmitry G. Chechin, ${ }^{1,3}$ Christof Lüpkes, ${ }^{2}$ Irina A. Repina, ${ }^{1,3}$

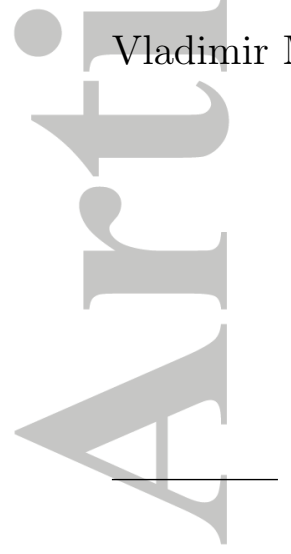

D.G. Chechin, A.M. Obukhov Institute of Atmospheric Physics, 119017, Pyzevsky 3, Moscow,

Russia. (chechin@ifaran.ru)

${ }^{1}$ A.M. Obukhov Institute of Atmospheric

Physics, Russian Academy of Sciences,

Moscow, Russia

${ }^{2}$ Alfred Wegener Institute for Polar and

Marine Research, Bremerhaven, Germany

${ }^{3}$ Russian State Hydrometeorological

University, St. Petersburg, Russia

This article has been accepted for publication and undergone full peer review but has not been through the copyediting, typesetting, pagination and proofreading process, which may lead to differences between this version and the Version of Record. Please cite this article as doi: 10.1002/jgrd.50679

(C)2013 American Geophysical Union. All Rights Reserved. 
Abstract. A non-hydrostatic model (NH3D) is used for idealized dry quasi2D simulations of Arctic cold-air outbreaks using horizontal grid spacings between 1.25 and $60 \mathrm{~km}$. Despite the idealized setup, the model results agree well with observations over Fram Strait. It is shown that an important characteristic of the flow regime during CAOs is an ice-breeze jet with a maximum wind speed exceeding often the large-scale geostrophic wind speed. According to the present simulations, which agree very well with those of another non-hydrostatic mesoscale model (METRAS), the occurrence, strength, and horizontal extent $L$ of this jet depend strongly on the external forcing and especially on the direction of the large-scale geostrophic wind relative to the orientation of the ice edge. The latter dependency is explained by the effects of the thermally induced geostrophic wind over open water and Coriolis force. It is found that coarse-resolution runs underestimate the strength of the jet. This underestimation has important consequences to the surface fluxes of heat and momentum, which are also underestimated by about 10$15 \%$ on average over the region between the ice edge and 120-180 km downstream. Our results suggest that a grid spacing of about $L / 7$ is required (about 10-30 $\mathrm{km}$ ) to simulate the IBJ strength with an accuracy of at least $10 \%$. Thus the results of large scale models as well might contain uncertainties with regards to the simulated IBJ strength which would influence the energy budget in a large region along the marginal sea ice zones.

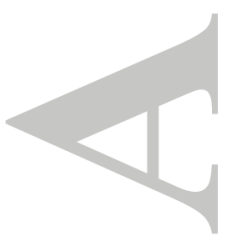




\section{Introduction}

Marine cold-air outbreaks (CAOs) are a typical meteorological phenomenon occurring

during the cold seasons in the polar regions of both hemispheres. During such events, cold air originating from ice or snow covered regions is transported over large distances while it is heated by the warm ocean surface. Close to the sea-ice edge, sensible heat fluxes can amount during CAOs up to $650 \mathrm{Wm}^{-2}$ [Brümmer, 1996] and values can be still above $100 \mathrm{Wm}^{-2}$ several hundred kilometers downstream. This leads to a large heat loss of the ocean and in some regions, such as the Labrador and Greenland Seas, cooling and increase of salinity in the upper ocean layers may contribute to deep convection in the ocean [Marshall and Schott, 1999; Gryanik et al., 2000; Pagowski and Moore, 2001]. This characterizes CAOs as a factor that might have a significant influence on both regional and global climate. Furthermore, during CAOs, small-scale processes in ocean and sea ice (like sea-ice drift, freezing or melting, upwelling along the ice edge) play an important role for the marginal sea ice zone (MIZ) dynamics, as described by Guest et al. [1995a; 1995b], and in a preconditioning phase of deep convection events [Häkkinen, 1987; Roach et al., 1993]. Thus an adequate simulation of air-sea-ice interaction over the MIZ during CAOs in both global and regional climate models is highly desirable.

Although CAOs can be resolved to some extent by global circulation models and regional climate models [Kolstad and Bracegirdle, 2008], it can be expected that the coarse spatial resolution does not yet allow an explicit reproduction of the full range of mesoscale variability of meteorological parameters during CAOs. This might in turn affect the accuracy of the simulated energy exchange between atmosphere and ocean. Deficiencies of 
large scale models can be expected, especially, in the region with the largest atmospheric variability which is the region between the ice edge and about $300 \mathrm{~km}$ downstream [Guest et al., 1995a]. There, horizontal gradients of wind and temperature as well as surface heat fluxes during CAOs are largest. Due to the warming of cold air masses, a convective boundary layer is rapidly growing while wind speed and surface stress are increasing [Brümmer, 1996].

The growth of wind speed over the open water was discussed in several studies. In some of them [Overland et al., 1983; Langland et al., 1989; Guest et al., 1995b] one of the proposed reasons for it was the baroclinicity related to the surface heating over open water. This mechanism is often referred to as an ice-breeze circulation (IBC). Numerical simulations [Overland et al., 1983; Reynolds, 1984; Lüpkes and Schlünzen, 1996] showed a possible existence of a wind speed maximum at a distance of 50-150 km from the ice edge with wind speeds about $10 \%$ higher than further downstream. This regime will be called an ice-breeze jet (IBJ) in the following.

Brümmer [1996] observed 10 cases with CAOs over Fram Strait northwest of Svalbard and found in four cases the occurrence of an IBJ about $100 \mathrm{~km}$ downwind of the MIZ, which he explained by an IBC being imposed on a large-scale flow. A study by Kolstad [2008, his Figure 2] gives a hint on the existence of an IBJ even in seasonally averaged data. Based on QuikSCAT satellite observations, he found some statistical evidence of a wind speed increase along the Fram Strait MIZ extending from the north-west of Svalbard to the south of Greenland, about 100-150 km wide, and being most pronounced in winter

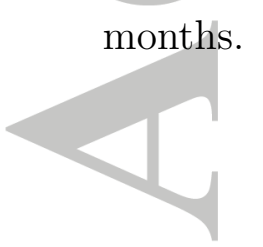

(C)2013 American Geophysical Union. All Rights Reserved. 
However, it seems that an IBJ which strongly affects the energy fluxes doesn't exist always during CAOs. So, the above mentioned observations by Brümmer [1996] show also that in the remaining cases no wind speed maximum was found. Moreover, results of a modeling and observational study by Wacker et al. [2005] of a CAO over Fram Strait also do not show the existence of a wind maximum. Fairall et al. [1987] argue that the acceleration of wind caused by baroclinicity in the atmospheric boundary layer (ABL)

might be cancelled sometimes by the decelerating effect of the sloping inversion at the ABL top.

Numerous studies of CAOs based on observations, modeling experiments, and theoretical analyses addressed a number of further questions such as the heat budget of the advected air mass [Brümmer, 1997], parameterizations of turbulent exchange [Chrobok et al., 1992; Lüpkes and Schlünzen, 1996; Gryanik and Hartmann, 2002; Gryanik et al., 2005], the effect of cloud microphysics on turbulent exchange [Harrington and Olson, 2001], the effect of a realistic representation of the sea ice margin in models simulating the convective boundary layer (CBL) development [Pagowski and Moore, 2001; Lüpkes and Birnbaum, 2005; Wacker et al., 2005; Liu et al.,2006], as well as observations and simulations of roll-like convective structures [Hartmann et al., 1997; Renfrew and Moore, 1999; Liu et al., 2004; Liu et al., 2006; Maesaka et al., 2006; Gryschka et al., 2008] and also their theoretical investigation [Etling and Brown, 1993; Young et al., 2002]. However, a systematic study of IBJ characteristics has never been performed for a wide range of meteorological conditions and thus forms the first goal of the present work. We will quantify the IBJ strength and horizontal extent for different absolute values of the large-scale 
geostrophic wind, its direction relative to the ice edge orientation, temperature difference between open water and sea ice, and the ABL height over sea ice.

Another topic which has not yet been studied is the effect of horizontal grid spacing of regional climate models on the simulated meteorological variables and their spatial variability during CAOs. This topic, which is important for climate modeling, forms the second main goal of the present work. We aim here to quantify the effect of horizontal resolution, especially on the simulated near-surface vertical fluxes of heat and momentum.

This investigation is important for two reasons. The first is that it will help to identify specific uncertainties of results from climate modeling in polar regions which have not yet received much attention in the literature. The second one is related to the very large values of vertical energy fluxes during CAOs, which drive water mass modification due to intensive cooling, the related sea ice formation and vertical mixing. Although deep ocean convection occurs typically farther south, these processes result in a modification of temperature and salinity in the $\mathrm{CAO}$ region and are thus important for the regional circulation in the ocean [Mauritzen, 1996].

Our strategy described in more detail in the next section is to use a non-hydrostatic mesoscale model (NH3D) to simulate CAOs over Fram Strait with different horizontal grid spacing which is, however, always much larger than that of Large Eddy Simulation (LES) and cloud-resolving models. This means that we cannot resolve convective thermals and rolls. The corresponding turbulence caused by the strong convection is treated by the subgrid closure. With this strategy, which has been used in many previous investigations by others [e.g. Wai and Stage, 1989; Bechtold et al., 1992; Glendening, 1994; Vihma et al., 2002; Savijarvi, 2012], the flow can be considered as quasi-2D since the remaining 
ice edge-parallel gradients are small. Other simplifying assumptions are applied as well (Section 2), so that the CAO simulation is idealized and conclusions should be considered in the light of this idealization. The restriction to the mesoscale allows us to test the sensitivity on a large parameter set with manageable costs (CPU time).

We start with the simulation of an observed cold-air outbreak using $1.25 \mathrm{~km}$ and $5 \mathrm{~km}$ horizontal grid spacings (Section 3). This serves as a reference run and helps verifying the NH3D model which has never been used before to simulate processes in the Arctic atmosphere. The first runs aim also to study the sensitivity of the model on the MIZ width. The reference run is repeated then using a coarse horizontal resolution of $60 \mathrm{~km}$ (Section 5), which is similar to the grid spacing often used in regional climate models and in some reanalyses as in ERA-Interim. Moreover, some of the results of NH3D are compared with those of another nonhydrostatic model (METRAS [Schlünzen, 1990]) (Appendix B), which was successfully used in the past to simulate many different meteorological regimes in the Arctic [e.g., Lüpkes and Schlünzen, 1996; Vihma et al., 2003; Birnbaum and Lüpkes, 2002; Dierer et al., 2006; Lüpkes et al., 2008b]. This comparison shows that the present results are not specific for one model but can be seen as representative for regional models. The study is accomplished by a series of idealized model experiments with varying external parameters and horizontal resolution (Section 6-7). The range of parameters considered is typical for wintertime CAOs in high latitudes, but is wider than in previous studies. Moreover, we systematically investigate the dependence of mesoscale features of CAOs on the wind direction, which was not considered before. The sensitivity of the IBJ magnitude on the wind direction is qualitatively explained by estimating the local geostrophic wind in the ABL over open water. It is shown that the latter is a good 
estimate of the actual wind speed at distances of about $200 \mathrm{~km}$ and further downstream from the ice edge.

\section{Modelling Background}

\subsection{The NH3D Model}

NH3D is a non-hydrostatic model based on a set of equations by Miller and White [1984] utilizing vertical $\sigma$-coordinates. It has been successfully applied for simulating orographic gravity waves, generated over a two- and three-dimensional relief [Miranda and James,

1992; Miranda and Valente, 1997], as well as for breeze circulations over Western Siberia [Stepanenko et al., 2008]. The model consists of prognostic equations for the three wind components, potential temperature and surface pressure. A diagnostic elliptic equation is solved for the geopotential perturbation.

A staggered Arakawa-C grid is used with constant grid spacing in horizontal directions, while the vertical grid spacing is increasing with height. Advection is calculated by a second-order centered scheme, using a flux-corrected transport algorithm [Zalesak, 1979] to maintain monotonicity. The leap-frog scheme is used for integration in time with a RobertAsselin time filter applied at each time step. Horizontal numerical filtering is represented by a monotonic forth-order diffusion scheme with a flux-limiter proposed by Xue [2000]. At the northern inflow boundary, fixed boundary conditions are used for the wind speed components and for the potential temperature. At the other boundaries, zero-gradient boundary conditions are used for the boundary-parallel wind components and potential temperature. For the boundary-normal wind velocity, radiation boundary conditions are used by applying the Orlanski scheme [Orlanski, 1976] extended by Raymond and Kuo [1986]. 
NH3D is forced by a constant in time and space large scale pressure gradient that corresponds to a geostrophic wind speed far away from the sea ice edge.

Monin-Obukhov similarity theory serves to parameterize turbulent fluxes in the surface

layer. Businger-Dyer universal functions are used in case of unstable stratification and log-linear universal functions [Dyer, 1974] in neutral and stable conditions. When the sea ice concentration $A$ across the MIZ is within the interval $(0,1)$ surface fluxes over ice and water are calculated separately and are then averaged according to their surface fraction. The roughness length for momentum $z_{0}$ is set to $0.001 \mathrm{~m}$ over sea ice and is calculated according to the Charnock formula $z_{0}=\alpha u_{*}^{2} / g$ over open water where $u_{*}$ is the friction velocity and $\alpha=0.0185$ is used according to $W u$ [1980]. The roughness length for scalars is set to $0.1 z_{0}$

Turbulent fluxes above the surface layer are parameterized by a combination of a local closure above the ABL and a nonlocal turbulence closure within the ABL as described in Lüpkes and Schlünzen [1996]. We assume that the vertical heat transport by large convective eddies is fully parameterized by the nonlocal closure. The latter is based on Holtslag and Moeng [1991] and was designed specifically to account for the nonlocal vertical transport of heat by large eddies (see next subsection). It has been verified for different $\mathrm{ABL}$ regimes in a wide range of conditions which can be classified in terms of the parameter $z_{i} / L$ where $L$ is the Obukhov length scale. The above mentioned modified version of the Holtslag and Moeng closure, used here, was successfully applied by Lüpkes and Schlünzen [1996] in conditions with rolls $\left(-z_{i} / L \approx 30\right)$ for the same case of CAO as considered here. Holtslag and Moeng [1991] based their closure on LES results for convective boundary layer with $-z_{i} / L \approx 10$. Moreover, Brown [1996] has shown that the 
Holtslag and Moeng closure is well reproducing a baroclinic convective boundary layer $\left(-z_{i} / L\right.$ varied from 0 to 26.1). Ayotte et al. [1996] demonstrated a good performance of this closure for both barotropic and baroclinic conditions with $-z_{i} / L$ of up to 18 . Lock [2000] successfully applied a modified Holtslag and Moeng closure, which is used now in the U.K. Met. Office Unified Model for cloud-topped boundary layer simulations.

Similar turbulence closures are used in weather prediction and regional climate models

(for example, the Troen and Mahrt [1986] nonlocal closure is used in MM5 [Pagowski and Moore, 2001]).

\subsection{Modelling Strategy}

For the present study, we use a horizontal grid spacing which is not resolving convective structures developing in CAOs. Since these structures are important for the transport of energy, we discuss in the following the consequences of this strategy and its relation to Large Eddy Simulations (LES) and observations.

Previous studies have shown that CAOs are a complex phenomenon, where processes of different spatial and temporal scales occur simultaneously. Small scale convective thermals and plumes are generated at the surface and penetrate through the whole boundary layer up to the inversion. Often, the convective plumes are organized in roll-like coherent structures, whose aspect ratios might vary in a wide range (from 2 to 15) [Etling and Brown, 1993; Atkinson and Zhang, 1996; Young et al., 2002]. It has also been shown that plumes and roll-like eddies can transport considerable amounts of heat and momentum [Brümmer, 1999] and are thus responsible for the nonlocal character of vertical turbulent exchange within the ABL. An explicit simulation of such a multi-scale system requires a very high resolution in the order of $50 \mathrm{~m}$ [Gryschka et al., 2008; Sullivan and Patton, 
2011]. In our present study, the focus is, however, on the mesoscale flow structure in CAOs and on its reproduction by models using grid sizes being typical for regional climate and weather forecast models. This means that we parameterize all the smaller scale turbulent convective processes, including thermals, plumes and rolls by the nonlocal turbulence closure mentioned above.

When the results of the mesoscale model are compared with observations or highresolution LES results, it is important to keep in mind that the mesoscale model assumes volume, time, and ensemble averaging of the Navier-Stokes equations as described by Cotton and Anthes [1989] (see their chapter 3). The appropriate length/time scales of the averaging operator are usually larger than the grid sizes and time steps of the model. A comparison with observational or LES data requires the same averaging of both data.

For aircraft or point measurements, the ensemble part of the averaging is usually substituted by spatial or temporal averaging assuming that the ergodicity condition is satisfied. For a convective ABL during CAOs, this requires horizontal averaging over a distance containing several wavelengths of rolls, which is about $50 \mathrm{~km}$ in the ice edge parallel direction (where $z_{i}$ and other CBL characteristics can be assumed as constant). We stress that for the treatment of turbulence as subgrid, model grid sizes can be much smaller than this averaging length (Cotton and Anthes [1989]). It is necessary only that the turbulent mixing produced by the subgrid-scale closure is efficient enough to account for mixing also by the large turbulent eddies. This is discussed in detail by Wyngaard [2004]. In our case, a grid cell of $1-5 \mathrm{~km}$ width is appropriate.

It is furthermore important to understand that, since nonlocal closures parameterize the whole turbulent transport, convective plumes should not be resolved. Thus the vertical 
velocity simulated by a mesoscale model in a convective ABL can be used as an indicator for the appropriate grid size. Honnert et al. [2012] showed by comparing mesoscale and high resolution simulations that when all convective motions are treated as subgrid, the modelled vertical velocity $w$ does not exceed values related to the mesoscale resolved structures. Values of $w$ are then in the range of $\mathrm{cm} \mathrm{s}^{-1}$ and are thus much smaller than vertieal velocities in plumes. One can conclude furthermore from their model results with

$\Delta y=1 \mathrm{~km}$ that the overall structure of the convective ABL was reproduced fairly well with a nonlocal mass-flux closure although this resolution produced only small vertical velocities.

To summarize, with our present approach we are not aiming to explicitly resolve convective motions which are parameterized by the turbulence closure. Previous studies of convective boundary layers showed that this causes some uncertainty in the results, however, the present findings can give important hints for future more detailed studies using LES. It shows furthermore the typical behavior of non-eddy resolving models like climate and weather prediction models when they are applied to CAOs, e.g., in different horizontal resolutions.

\subsection{Further Idealizations}

Radiation and microphysics are not considered in this study for simplicity. Modelling studies of CAOs with and without clouds by Wacker et al. [2005] and Lüpkes et al. [2012] show that the inclusion of cloud microphysics in a mesoscale model didn't have a large effect on the simulated fields of wind and temperature in CAOs. A possible reason is the high Bowen ratio often observed in Arctic CAOs. For example, during the campaigns called the Radiation and Eddy Flux Experiment (REFLEX) [Kottmeier et al., 1994] and 
the Arctic Radiation and Turbulence Interaction Study (ARTIST) [Hartmann et al., 1999; Lüpkes et al.,2012] the near-surface latent heat fluxes amounted to only $25 \%$ of the sensible heat flux. These numbers agree with measurements by Renfrew and Moore [1999] who found Bowen ratios of about 3-5 during a wintertime CAO over the Labrador Sea for even higher air temperatures $\left(-20^{\circ} \mathrm{C}\right)$ than the observed ones during the $\mathrm{CAO}$ considered here $\left(-30^{\circ} \mathrm{C}\right)$. They estimated the possible amount of latent heat release during condensation

to about $10 \%$ of the surface heat fluxes.

These findings do not mean that clouds have no impact, but this impact occurs mainly either on small scales, which affect the general development of the convective boundary layer structure only slightly, or far from the ice edge. Thus Müller and Chlond [1996] and Schröter et al. [2005] stress the influence of the latent heat release due to condensation upon the broadening of the convective cells far downstream from the ice edge. Olsson and Harrington [2001] find from their high resolution 2D model that radiative cooling and warming at the cloud top and base as well as latent heat release influence significantly the turbulent kinetic energy. However, the agreement of their model results with observations was in a similar quality as that found by Lüpkes and Schlünzen (1996) in their model runs without clouds. Also Gryanik and Hartmann [2002] find that statistics of turbulence obtained from an observed CAO with a cloudy convective ABL were similar to those obtained with a "dry" LES.

It cannot be excluded, however, that there are also situations with a stronger impact of clouds. Brümmer [1997] shows some cases in which the latent heat release due to condensation and sublimation can be dominant, but only at distances more than $300 \mathrm{~km}$ from the ice edge which are not considered in our study. Nevertheless, future studies 
should extend the present ones to less idealized situations and the conclusions of the present work should be considered in the light of our idealized assumptions.

\subsection{Representation of the Marginal Sea Ice Zone}

The development of the CBL is driven by a prescribed transition of the surface from cold and rough sea ice in the northern part of the domain to relatively warm and smooth open water in the southern part. The two surface types are separated by the MIZ. We define the latter here as the transitional zone from pack ice to open water where ice floes are broken and have diameters smaller than $1 \mathrm{~km}$. To represent the MIZ in the NH3D

model, the sea ice concentration $A$ is prescribed to change from north to south according

to

$$
A=\frac{1}{2}-\frac{1}{2} \tanh \left(\frac{y-y_{c}}{L_{0}}\right)
$$

where $y_{c}$ is the position of the MIZ center with $A=0.5$. This is also the position of the largest gradients of $A$ in north-south direction. By setting $\left(y-y_{c}\right)$ in the above equation to $L_{0}$, we obtain $L_{0}$ as half of the width of the zone where $0.12 \geq A \geq 0.88$. When we define the MIZ as the zone with $0.05 \geq A \geq 0.95$, its width $L_{M I Z}$ can be expressed through $L_{0}$ by setting $\mathrm{A}$ in Equation 1 to 0.05 and 0.95 . This results in $L_{M I Z} \approx 3 L_{0}$. An instantaneous jump from $A=1$ to $A=0$, which we use in most of the model runs to describe the abrupt ice edge, corresponds in the above equation to the limit $L_{0} / \Delta y \rightarrow 0$, where $\Delta y$ is the grid spacing of the model (later in the text we use $L_{M I Z}=0$ to refer to this case).

We stress that the representation of the MIZ as described above is a further strong idealization of reality in our study. For example, in nature there is a large variability of possible sea ice patterns in the MIZ so that the actual change of sea ice concentration 
as function of distance to the pack-ice may differ strongly from conditions prescribed by equation (1). Furthermore, in the present work, the increase of drag coefficient over the MIZ [e.g., Mai et al., 1996; Birnbaum and Lüpkes, 2002; Lüpkes and Birnbaum, 2005; Andreas et al., 2010; Lüpkes et al., 2012] is not taken into account. However, the present focus is not on the small scale atmospheric processes over the MIZ and the simplified model is sufficient. But it should be kept in mind that especially the results concerning

momentum fluxes (see section 3.3) would be modified in the MIZ region by an inclusion of form drag effects caused by floe edges.

Since the surface characteristics are prescribed to vary only in north-south direction and the impact of $3 \mathrm{D}$ convective cells on turbulence is parameterized, we can treat the CAOs as quasi-2D flow regimes by using only few grid points in the east-west direction, which is advantageous concerning required computer resources. Although this is an idealization of sea-ice conditions in nature, this provides a useful framework as it was often used to study sea breezes [e.g. Mahrer and Pielke, 1977; Bechtold et al., 1991].

\section{Reference Run}

NH3D has not yet been applied to Arctic regions. Thus our first step is to compare results of a simulated CAO over the northwestern Fram Strait with observations based on the aircraft campaign REFLEX II [Kottmeier et al., 1994]. The latter served already as a basis for the validation of the mesoscale model METRAS and its nonlocal turbulence closure [Lüpkes and Schlünzen, 1996] which is used now also in NH3D. We discuss in the following this CAO simulation, which will furthermore serve as a reference run for the later investigation of IBJ characteristics and of the impact of resolution. 
The meteorological conditions during the observed CAO were characterized by a large temperature difference of about $30 \mathrm{~K}$ between the air advected from north and the surface of open water as being typical during strong CAOs over the Fram Strait MIZ during the cold seasons. A satellite image (Figure 10 in Kottmeier et al., [1994]) shows a shallow stratocumulus cloud cover over open water south from the pack ice being also typical for CAO conditions and which is indicating the development of a convective boundary layer.

Dropsondes released from the Alfred Wegener Institute aircraft Polar 4 on 4th of March 1993 provide initial conditions and an estimation of surface temperatures. The observed conditions remained almost stationary for many hours and the CAO lasted over several days.

According to the dropsonde observations, the ABL consisted over ice of a shallow mixed layer capped by a strong inversion at about $150 \mathrm{~m}$ height (Fig.1, panel $a$, profile $a$ ). At a distance of $58 \mathrm{~km}$ north from the ice edge, the surface pressure amounted to 1027 hPa. Further downstream from the ice edge, the observations document the growth of a convective boundary layer (Fig.1, panel a) whose height was gradually increasing up to about $1500 \mathrm{~m}$ at $240 \mathrm{~km}$ distance from the ice edge.

Wind information is available from the drop sondes, but one should keep in mind that at that time the global positioning system (GPS) was not yet available for radiosondes and the accuracy of the Omega wind finding system (see Govind [1975]), especially at low levels was thus lower than nowadays. This means that the high wind speed values close to the surface (Fig.1, panel b) might also be an artefact caused by limited vertical resolution of the wind determination technique. According to these measurements, the ABL wind regime was characterized by a low-level jet (Fig.1, panel b) over the open water region 
with a maximum wind speed of up to $18 \mathrm{~ms}^{-1}$. The geostrophic wind above the ABL as estimated from the dropsondes (average at $2000 \mathrm{~m}$ height) was from northwest with $U_{g}$ $=6.2 \mathrm{~ms}^{-1}$ and $V_{g}=9.4 \mathrm{~ms}^{-1}$, where $U_{g}$ is the west-east component and $V_{g}$ the southnorth component. The positive directions of the $\mathrm{x}$ - and $\mathrm{y}$-axes are from east to west and from north to south, respectively (see Figure 6). This results in $\left|\mathbf{V}_{\mathbf{g}}\right|=11.26 \mathrm{~ms}^{-1}$ and $\alpha=33.4^{\circ}$, where $\alpha$ is the angle between $\left|\mathbf{V}_{\mathbf{g}}\right|$ and the direction orthogonal to the ice edge (see Figure 6). We use furthermore aircraft measurements by Brümmer [1996] which were obtained in the same CAO by another aircraft using a 5-hole probe for wind measurements. According to the aircraft vertical sounding over the ice edge (see Figure 5 of Brümmer [1996]) the absolute value and direction of the large-scale geostrophic wind agreed well with those obtained from the dropsondes. Near-surface wind speed measurements of Brümmer are available at several positions in the convective ABL as shown in his Figure 3. It is important that these data were obtained by spatial averaging of instantaneous measurements during $50 \mathrm{~km}$ flight legs parallel to the ice edge. They indicate an increase of absolute wind speed to about $15 \mathrm{~ms}^{-1}$ at a distance of about $150 \mathrm{~km}$ from the ice edge (Figure 2). Further downwind the wind speed is decreasing and then increasing again at a distance of about $400 \mathrm{~km}$. Based on both data sets from drop sondes and aircraft we thus conclude that the ice-breeze jet was present during this CAO.

The initialization procedure of NH3D is the same as was described in the study by Lüpkes and Schlünzen [1996] for the model METRAS. The profiles measured over the sea ice cover in the northern part of the domain (Fig.1, panel $a$, profile $a$ ) are used to force a 1D version of NH3D, whose steady state solution serve as initial conditions in the whole domain of NH3D. The surface temperature is prescribed to $-32{ }^{\circ} \mathrm{C}$ over ice and is assumed 
to change linearly over open water from the freezing point $-1.8^{\circ} \mathrm{C}$ close to the ice edge to $+3{ }^{\circ} \mathrm{C}$ at a distance of $300 \mathrm{~km}$ south from the ice edge. Both sea ice and open water surface temperatures are kept constant during the model runs.

The position of the ice edge and the width of the MIZ are not exactly known due to cloudy conditions during the REFLEX campaign. We prescribe a narrow MIZ with $L_{M I Z}$ equal to about $12 \mathrm{~km}$ in equation (1) and discuss later the possible effect by using much larger values.

A long and narrow model domain orientated in north-south direction is used with 400 $\mathrm{km}$ over sea ice and $400 \mathrm{~km}$ over open water. In east-west direction, only 6 grid points are used since the mesoscale flow can be treated as quasi-2D. Test runs show (not presented here) that the results do not depend on the domain width. This is due to the use of zero-gradient boundary conditions for scalars and boundary-parallel wind components. Another reason is that plumes are not resolved with the used grid size.

We consider in this section two different horizontal grid spacings $\Delta y$. First, $\Delta y=$ $1.25 \mathrm{~km}$ is used, which allows resolving a narrow MIZ. In another run, we prescribe $\Delta y$ $=5 \mathrm{~km}$, which is similar to the grid spacing used in previous studies of CAOs [Lüpkes and Schlünzen, 1996; Pagowski and Moore, 2001]. The vertical grid consists in all runs of 47 levels and grid spacing is gradually increasing with height starting from about $30 \mathrm{~m}$ near the ground to $100 \mathrm{~m}$ at $1 \mathrm{~km}$ height. Perhaps surprisingly, the use of higher vertical resolution causes only very little changes of the results (e.g., less than $2 \%$ for the absolute wind speed in the ABL).

The model is run over 60 hours until a quasi-stationary state is achieved. After 60 hours of integration the temporal variation of the simulated surface fluxes is not larger 
than about $1 \%$. The steady state profiles are then compared with the dropsonde observations and aircraft measurements described above. A quantitative comparison against dropsonde observations is useful only for temperature because of the large uncertainty of the wind data from the drop sondes. One of the reasons for this uncertainty has already been discussed. Another reason is that drop sonde data represent always instantaneous measurements while model results refer to mean quantities. This difference is more important for wind than for temperature due to their different variability in a convective boundary layer. Previous investigations [e.g. Gryanik and Hartmann, 2002, their figure 1] showed that for a typical CAO $\left(\left(\overline{w^{\prime} \theta^{\prime}}\right)_{s}=0.3 \mathrm{~ms}^{-1} \mathrm{~K}, w_{*}=2 \mathrm{~ms}^{-1}, z_{i}=1000 \mathrm{~m}\right)$ the

variance of the potential temperature is only about $0.2 \mathrm{~K}^{2}$ while the variance of horizontal velocity is much larger, namely $1.2-1.6 \mathrm{~m}^{2} \mathrm{~s}^{-2}$ in the center of the ABL. Thus the dropsonde wind has a large uncertainty.

\subsection{Comparison of NH3D with Observations}

Figure 1 shows the steady state results of the NH3D model after 60 hours of integration for $\Delta y=1.25 \mathrm{~km}$ and dropsonde observations from the 4th of March. Obviously, both the growth of the ABL height and the gradual heating of the advected cold air mass over open water is modeled in good agreement to the observations. The degree of agreement is similar as in the study by Lüpkes and Schlünzen [1996] based on the model METRAS. The observed boundary layer is slightly warmer in its upper part as compared to the simulation. Discrepancies with observations can be attributed to diabatic heating connected with clouds, which were neglected in the current study.

The simulated wind speed at $90 \mathrm{~m}$ height is shown together with aircraft observations from Brümmer [1996] in Figure 2. Both simulation and observations show an acceleration 
(IBJ) of roughly $3 \mathrm{~ms}^{-1}$ downstream of the ice edge. Also the observed horizontal scale of the IBJ is well reproduced by the model but in the first $120 \mathrm{~km}$ at lower wind speeds. The observations by Brümmer demonstrate another increase of wind speed farther downwind at a distance of about $400 \mathrm{~km}$ from the ice edge. Since this effect is only visible at one point, it might have been caused by the large-scale synoptic forcing or at this latitude also by the orography of Svalbard. But future investigations, e.g., with LES or future observations might help to explain if this hints to a weakness of the mesoscale simulation. An increase of wind, discussed in more detail in the next subsection, is also found in the observations from dropsondes (Figure 1). Simulated values (Figures 2, 3) are smaller than those obtained by the dropsondes. The difference is especially large below $200 \mathrm{~m}$ (up to $5 \mathrm{~ms}^{-1}$ ), where the uncertainties of the dropsondes are, however, the largest so that this difference should not attain much attention.

\subsection{D Structure of Simulated Fields with $\Delta y=1.25 \mathrm{~km}$ and $\Delta y=5 \mathrm{~km}$}

Figures 3 and 4 show cross-sections of the simulated mean fields and turbulent fluxes after 60 hours of integration using $\Delta y=1.25 \mathrm{~km}$ and $\Delta y=5 \mathrm{~km}$. In these model runs, the surface temperature of open water is assumed to be at the freezing point throughout the domain so that the results differ slightly from those shown in Figure 1. In the following sections, the run with $\Delta y=5 \mathrm{~km}$ is further referred to as the reference run.

The Figures 3 and 4 document that the results of the model runs with different resolution agree very well. The main feature of the simulated fields consists in the growth of a convective ABL over the open water, which is clearly seen in the potential temperature $\theta$. Results of both runs show that $\theta$ increases slightly with height in the upper part of the ABL, which is typical for convective conditions. In a dry environment, this is only possible 
with a nonlocal turbulence closure allowing countergradient fluxes of heat generated by large plumes. This has been demonstrated earlier for CAOs by others [e.g., Chrobok et al., 1992; Lüpkes and Schlünzen, 1996; Noh et al., 2003].

Another important feature, which is similarly reproduced with both resolutions, is the simulated absolute horizontal wind speed. In both runs the maximum within the ABL is about $13.6 \mathrm{~ms}^{-1}$ in a distance of about $150 \mathrm{~km}$ downstream from the ice edge. This value exceeds the large-scale geostrophic wind speed by roughly $2.5 \mathrm{~ms}^{-1}$. So, the presence of an ice-breeze jet (IBJ) is clearly pronounced here, as it was the case in 4 of 10 CAOs observed by Brümmer [1996] in the Fram Strait region.

NH3D produces sinking motions over the ABL close to the ice edge in the order of several $\mathrm{cm} \mathrm{s}^{-1}$. Rising motions within the ABL occur further downstream and have the same magnitude as sinking motions. The reason for this structure of the vertical wind field, which is also very similar in both runs, is a horizontal divergence of the horizontal wind within the ABL. Both the magnitude and spatial pattern of vertical motions are similar to those derived by Brümmer [1997] (his Table 2) based on mass balance estimates for 10 CAOs.

Figure 3 shows that with $\Delta y=1.25 \mathrm{~km}$ and $\Delta y=5 \mathrm{~km}$ the spatial structure and absolute values of the vertical velocity $w$ are very similar. Only above the ABL, $w$ becomes more noisy in the run with smaller grid spacing while the magnitude of $w$ remains still in the order of centimeters. This value is much smaller than local values in convective plumes and rolls during CAOs. This demonstrates that turbulent convective motions are neither simulated explicitly with $\Delta y=5 \mathrm{~km}$ nor with $\Delta y=1.25 \mathrm{~km}$.

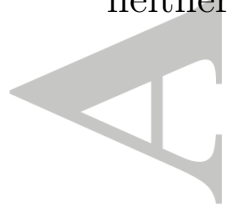


Figure 3 shows also the mesoscale pressure gradients $(\partial p / \partial y)_{\text {meso }}$ defined as the difference between the total horizontal pressure gradient and its large scale part. The latter is prescribed as a constant external forcing. Negative values of $(\partial p / \partial y)_{m e s o}$ over open water indicate a decrease of $p$ with distance from the ice edge. This is due to the heating of the advected air mass and the corresponding horizontal temperature gradient. Such a pattern with low pressure over the open water and higher pressure over ice is typical for CAOs $\left[\right.$ Brümmer, 1996]. $(\partial p / \partial y)_{m e s o}$ has a minimum close to the ice edge at the lowest model level with values less than $-2 \mathrm{~Pa} \mathrm{~km}^{-1}$ and its absolute value is decreasing further downwind.

Figure 4 shows the simulated turbulent fluxes of sensible heat and momentum. Maximum heat fluxes occur in the surface layer next to the ice edge with values exceeding $600 \mathrm{Wm}^{-2}$. The simulated momentum fluxes show two maxima, one at the surface and another one in the upper part of the ABL.

To summarize, the results of our idealized simulations reproduce general features of CAOs, which are known from previous observations. Moreover, there is a very good agreement between the results of NH3D and those of the model METRAS, as shown in Appendix B. Since results obtained with $\Delta y=1.25 \mathrm{~km}$ and $\Delta y=5 \mathrm{~km}$ differ only slightly from each other, we use model runs with $\Delta y=5 \mathrm{~km}$ in the following investigation as a reference for runs with lower resolution. This helps us to save CPU time with respect to the extensive sensitivity studies.

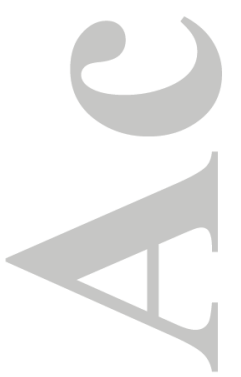




\section{Ice Breeze Characteristics}

\subsection{The Magnitude}

Before we proceed with the further investigation concentrating on the effect of model

resolution and external parameters, we introduce in this section criteria which will help to identify the IBJ and to quantify its main features - characteristic length scale and magnitude.

Based on Figures 3 and 5, the IBJ can be identified in the simulated fields when the magnitude $M$ for the normalized absolute wind speed maximum within the ABL satisfies the inequality

$$
M=\frac{\left|V_{\max }\right|-\left|\mathbf{V}_{\mathbf{g}}\right|}{\left|\mathbf{V}_{\mathbf{g}}\right|} \geq C_{M},
$$

where $\left|V_{\text {max }}\right|$ is the maximum absolute wind speed within the ABL and $\left|\mathbf{V}_{\mathbf{g}}\right|$ is the undis-

turbed geostrophic wind speed far away from the ice edge (see Figure 5). Equation 2 shows that $M$ is a measure of the relative contribution of the IBJ to the CAO regime. The threshold value $C_{M}=1 / 10$ is sufficient for our purposes. In the reference run we have $M=\left(13.5 \mathrm{~ms}^{-1}-11.26 \mathrm{~ms}^{-1}\right) / 11.26 \mathrm{~ms}^{-1} \approx 0.2 ;$ so the IBJ can be clearly identified. In the sensitivity studies described in section 6 , we show that for a certain range of meteorological parameters the inequality (2) is violated.

Another quantity that can be used for the analysis of the ice breeze is the geostrophic wind $\mathbf{G}_{\mathbf{m}}$ averaged over the ABL depth. The relation between $\mathbf{G}_{\mathbf{m}}$ and the actual wind speed in the ABL was well studied for a horizontally quasi-homogeneous baroclinic ABL [e.g. Arya and Wyngaard, 1975; Venkatesh and Danard, 1977; Brown, 1996; Sorbjan, 2004]. Therefore, we consider $\mathbf{G}_{\mathbf{m}}$ at some distance from the ice edge where horizontal 
inhomogeneities in the convective ABL become small. This is the case about $200 \mathrm{~km}$ south from the ice edge (Figure 5).

We consider in the following the fraction $\left|\mathbf{G}_{\mathbf{m}}\right| /\left|\mathbf{V}_{\mathbf{g}}\right|$. We remind that $V_{g}$ is constant

in space and time and represents the large-scale barotropic forcing of NH3D. Under a number of assumptions (see Appendix A) $\left|\mathbf{G}_{\mathbf{m}}\right| /\left|\mathbf{V}_{\mathbf{g}}\right|$ is given by

$$
\frac{\left|\mathbf{G}_{\mathbf{m}}\right|}{\left|\mathbf{V}_{\mathbf{g}}\right|}=\left[1+2 \frac{\mathbf{V}_{\mathbf{g t}} \cdot \mathbf{i}}{\left|\mathbf{V}_{\mathbf{g}}\right|} \sin \alpha+\left(\frac{\mathbf{V}_{\mathbf{g t}} \cdot \mathbf{i}}{\left|\mathbf{V}_{\mathbf{g}}\right|}\right)^{2}\right]^{1 / 2},
$$

where $\alpha$ is the angle between the direction of $\mathbf{V}_{\mathbf{g}}$ and the direction orthogonal to the ice edge (see Figure 6), and $\mathbf{V}_{\mathbf{g t}} \cdot \mathbf{i}=g z_{i} /\left(2 f \theta_{m}\right) \partial \theta_{m} / \partial y$ is the ABL-vertically averaged $x$-component of the thermally induced geostrophic wind as defined in Appendix A, where $\mathbf{i}$ is the unit vector in westward direction, and $\theta_{m}$ is the corresponding average potential temperature. Equation (3) shows that $\left|\mathbf{G}_{\mathbf{m}}\right| /\left|\mathbf{V}_{\mathbf{g}}\right|$ depends on both the angle $\alpha$ and the ratio $\left|\mathbf{V}_{\text {gt }}\right| /\left|\mathbf{V}_{\mathbf{g}}\right|$.

Using in (3) for all quantities the values at $200 \mathrm{~km}$ downstream of the ice edge from the reference run $\left(z_{i}=900 \mathrm{~m}, \theta_{m}=255 \mathrm{~K}, \partial \theta_{m} / \partial y=3.0 \times 10^{-5} \mathrm{Km}^{-1}\right.$ as well as $\left|\mathbf{V}_{\mathbf{g}}\right|=11.26 \mathrm{~ms}^{-1}, \alpha=33.4^{\circ}$ and $\left.f=1.432 \times 10^{-4} \mathrm{~s}^{-1}\right)$, we obtain $\left|\mathbf{G}_{\mathbf{m}}\right| \approx 12.8 \mathrm{~ms}^{-1}$ and $\left|\mathbf{G}_{\mathbf{m}}\right| /\left|\mathbf{V}_{\mathbf{g}}\right| \approx 1.14$. At this position, the difference between the geostrophic wind above the ABL and the average wind within the ABL as obtained from equation (3) is similar as the difference between modelled wind above the $\mathrm{ABL}$ and $\mathbf{V}_{\mathbf{m}}$ representing the actual modelled wind averaged over the ABL depth (see Figure 5).

However, the actual ABL-mean wind speed $\mathbf{V}_{\mathbf{m}}$ differs from $\mathbf{G}_{\mathbf{m}}$ at any position due to horizontal advection and Ekman turning. Far away from the ice edge, advection is small and we estimate only the Ekman effect. Under the assumptions of a quasi-stationary and horizontally homogeneous well-mixed ABL, as described in Byun and Arya [1986], 
the Ekman deceleration and turning is determined by the geostrophic Ekman number $E_{m}=C_{D}\left|\mathbf{G}_{\mathbf{m}}\right| /\left(f z_{i}\right)$, where $C_{D}$ is the drag coefficient. Inserting values from the reference run again at a distance of $200 \mathrm{~km}$ over the open water $\left(C_{D}=1.8 \times 10^{-3}\right)$, we obtain $E_{m}=0.19$. For such a value, the difference between the actual wind $\left|\mathbf{V}_{\mathbf{m}}\right|$ and $\left|\mathbf{G}_{\mathbf{m}}\right|$ is about 1-2\% only, according to Byun and Arya (their Figure 1). Therefore, Equation (3) provides an estimate of $\left|\mathbf{V}_{\mathbf{m}}\right|$ at distances about $200 \mathrm{~km}$ and larger downstream from the ice edge.

Due to the thermally induced $\mathbf{V}_{\mathbf{g t}}$, also the direction of $\mathbf{G}_{\mathbf{m}}$, which is $\phi=$ $\arcsin \left(\left|\mathbf{V}_{\mathbf{g}}\right| /\left|\mathbf{G}_{\mathbf{m}}\right| \sin \alpha+\left|\mathbf{V}_{\mathbf{g t}}\right| /\left|\mathbf{G}_{\mathbf{m}}\right|\right)$, becomes different from $\alpha$ (see Figure 6 ). Using again values at $200 \mathrm{~km}$ downstream of the ice edge from the reference run, we obtain $\phi \approx 46^{\circ}$. A comparison of this value with $\alpha=33.4^{\circ}$ shows that the ABL geostrophic wind $\mathbf{G}_{\mathbf{m}}$ turns to the right relative to $\mathbf{V}_{\mathbf{g}}$ by about $14^{\circ}$.

It is important to compare the turning of $\mathbf{G}_{\mathbf{m}}$ caused by baroclinicity with the Ekman turning. For $E_{m}=0.19$, the turning of the ABL-mean wind is about $10^{\circ}$ to the left relative to the direction of $\mathbf{G}_{\mathbf{m}}$ [Byun and Arya, 1986 (their Figure 1)]. The Ekman turning acts in opposite direction than that caused by $\mathbf{V}_{\mathbf{g t}}$ while the magnitudes of the two effects are similar (Figure 6). Therefore, in the range of parameters considered here, the direction $\beta$ of the vertically averaged flow in the convective ABL must be close to $\alpha$.

According to (3), one can expect an asymmetry of the simulation results relative to the direction orthogonal to the ice edge when the large scale geostrophic wind direction varies symmetrically, for example, for the cases $\pm \alpha=33.4^{\circ}$ (see Figure 6 ). Later, we show indeed (Section 6) that this asymmetric response of the wind direction in the convective 
ABL on a symmetric variation of $\alpha$ takes place has a strong impact on the simulated values of wind speed and surface fluxes.

\subsection{The Horizontal Scale}

Another important characteristic of the IBJ is its horizontal scale. The latter is chosen as the width $L$ of the IBJ which we define as

$$
L=\left|y_{\max }-y_{\min }\right|
$$

where $y_{\max }$ and $y_{\min }$ are the positions with extreme values of the horizontal gradients of $\mathbf{V}_{\mathbf{m}}$ (Figure 5).

Other horizontal scales related to the IBJ could be considered as well. One of them is the distance $l$ from the ice edge at which the maximum wind speed occurs. However, as we show later, in the range of parameters considered here, the use of $l$ does not lead to different findings than using $L$ as defined by equation 4. Glendening [1994] also studied the IBC and introduced another horizontal scale based on the Rossby deformation radius. However, Glendening [1994] considered ice edge parallel surface wind only.

Finally, we introduce a criterion that relates the necessary model resolution to the IBJ horizontal scale $L$. The IBJ can be resolved sufficiently well, when

$$
R=\frac{\Delta y}{L} \leq c_{L}
$$

where $\Delta y$ is the horizontal grid size and $c_{L}$ is a threshold value. We use $c_{L}=0.25$ in the following as atmospheric models are able to resolve only structures with horizontal scales larger than at least about $4 \Delta y[$ Walters, 2001]. For the reference case, the ratio $R$ is $5 \mathrm{~km} / 205 \mathrm{~km} \approx 0.025$. Since in the reference run $R=0.025 \ll 0.25$, we conclude that 
$\Delta y=5 \mathrm{~km}$ is enough to reproduce the IBJ. Equation (5) might not be satisfied for large

$\Delta y$ and also for small values of $L$ which can occur for certain external parameters.

\section{Effect of Horizontal Grid Spacing}

We compare the results of the reference run with $\Delta y=5 \mathrm{~km}$ in the following with a coarse-resolution run, for which we use a horizontal grid size $\Delta y=60 \mathrm{~km}$. The latter is similar to the horizontal grid spacing used in regional climate models for long-term climate studies [Kattsov and Källén, 2005; Tjernström et al., 2005] and also is the approximate horizontal resolution of the third generation reanalyses such as ERA Interim [Dee et al., 2011], MERRA (Modern-Era Retrospective Analysis for Research and Applications) [Rienecker et al., 2011] and CFSR (Climate Forecast System Reanalysis) [Saha et al., 2010]. As in the high-resolution run, we approximate the MIZ by an instantaneous jump in ice concentration from $A=1$ to $A=0$ from one grid cell to the next and consider

the ice edge to be located in the middle between two grid points. The results of the high-resolution run (Figure 3) are averaged over $60 \mathrm{~km}$ intervals and are then compared

to the results of the coarse-resolution run (Figure 7 ). The $2 \mathrm{D}$ vertical cross-sections of mean variables such as wind speed, temperature, and pressure gradient are considered, as well as turbulent fluxes of heat and momentum.

\subsection{Potential Temperature}

The largest differences between the potential temperatures of the high- and coarseresolution runs (panels $a 1$ and $a 2$ of Figure 7) occur within the ABL close to the ice edge. The potential temperature is overestimated in the coarse-resolution run by up to $2 \mathrm{~K}$. This leads to a decrease of the temperature difference between the temperatures at $10 \mathrm{~m}$ height 
and at the surface by about $10 \%$, which results in lower surface heat fluxes (Figure 9). The temperature overestimation is probably related to difficulties of the advection schemes to handle strong horizontal gradients of wind speed and temperature over the MIZ, when a coarse grid spacing is used. Test experiments showed that the use of a third-order upwind scheme [Hundsdorfer and Trompert, 1994] for temperature advection does not improve the results compared to the second-order centered-difference scheme as used by default in the NH3D model. The use of a first-order upwind scheme, however, makes the problem even worse and results in up to $3 \mathrm{~K}$ overestimation of temperature close to the ice edge.

\subsection{Magnitude and Direction of Horizontal Wind}

In the coarse-resolution run, the IBJ is also present (panels $b 1$ and b2 of Figure 7), but the maximum of wind speed is less pronounced than in the high-resolution run. The IBJ is underestimated by about $0.6 \mathrm{~ms}^{-1}$. This results in an $M$ value which is $25 \%$ smaller than that of the high-resolution run. This is expected, because with coarse grid spacing the resolution criterion given by equation (5) is not satisfied anymore $(R=$ $60 \mathrm{~km} / 205 \mathrm{~km}=0.29>0.25)$.

The spatial variability of the wind speed above the boundary layer is also underestimated by the model run with coarse resolution. So, above the ABL, a wind speed minimum at a distance of about $180 \mathrm{~km}$ from the ice edge and a maximum almost over the ice edge are not well reproduced by the coarse-resolution run.

The largest differences in the wind direction (panels $c 1$ and $c 2$ of Figure 7) occur close to the sea ice edge and amount up to about 3 degrees difference compared to the highresolution run. 


\subsection{Vertical Wind Speed}

Panels $d 1$ and $d 2$ of Figure 7 reveal that upward and downward motions are reproduced qualitatively well by the coarse-resolution run. However, the magnitude of vertical wind speed is underestimated by about $50 \%$. Vertical motions above the ABL might have an impact on the processes within the ABL. This has been discussed for a sea-breeze circulation by [Anthes et al., 1980; Anthes et al., 1982]. Thus, an underestimation of vertical motions related to the IBC might be a serious drawback.

\subsection{Mesoscale Horizontal Pressure Gradient}

The simulated field of $(\partial p / \partial y)_{\text {meso }}$ shows a minimum over open water close to the ice edge in both model runs (Figure 3 and panel $d 1$ of Figure 7 ) despite the differences in the resolution. But the minimum is underestimated by the coarse-resolution run (panel $d 2$ of Figure 7) by about $0.4 \mathrm{~Pa} \mathrm{~km}^{-1}$, which is around $25 \%$ of the absolute value of $(\partial p / \partial y)_{\text {meso }}$ resulting with high resolution. As the mesoscale pressure serves as the driving force for the IBC, its underestimation by the coarse-resolution run might be the main reason for the underestimation of the wind speed maximum over open water.

\subsection{Turbulent Fluxes of Heat and Momentum}

In the region between the ice edge and $200 \mathrm{~km}$ downstream the turbulent fluxes of momentum are strongly underestimated by up to $15 \%$ close to the surface when the coarse resolution is used (Figure 8). This is clearly related to the underestimation of the IBJ strength. The weaker IBJ as well as the less pronounced wind speed minimum above the ABL are also responsible for an underestimation of the momentum flux by the coarse-resolution run in the upper third of the ABL in the region between about 60 and 
$300 \mathrm{~km}$ distance from the ice edge. The reason for the underestimation is the reduced vertical wind shear when the wind maximum and minimum are less pronounced.

However, over the first grid cell downstream of the ice edge, there is a small area close to the top of the ABL where momentum fluxes are overestimated by the coarse-resolution run. This is due to an overestimation of the ABL thickness allowing mixing at larger heights.

Over open water, also the heat fluxes are underestimated by the coarse-resolution run in the region between the ice edge and $150 \mathrm{~km}$ downstream. The maximum underestimation occurs at the lowest model level close to the ice edge and amounts to about 15\%, which corresponds to $80 \mathrm{Wm}^{-2}$. This underestimation is caused by both temperature overestimation close to the ice edge and underestimation of the IBJ.

The underestimation of the surface fluxes of heat and momentum can be seen from Figure 9. The locations of the maximal fluxes of heat and momentum are not changed by the coarse resolution, but the maximal values obtained in the fine-resolution run and averaged over the $60 \mathrm{~km}$ intervals exceed those from the coarse-resolution run by about $15 \%$. It is interesting, that when $L_{M I Z}=45 \mathrm{~km}$ is prescribed in the high-resolution run (Figure 9 ), the simulated surface heat fluxes agree better with those of the coarse-resolution run where $L_{M I Z}=0 \mathrm{~km}$ is used. This means that a reduction of the resolution is to some extent equivalent to an increase of the MIZ width. This demonstrates the difficulties of coarse-resolution models to correctly simulate processes over sharp transitional zones. The latter can occur in nature, especially, at the onset of a CAO when the MIZ was compressed in the days before by an on-ice flow regime.

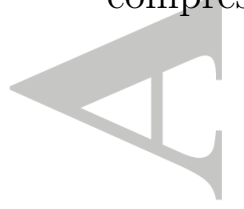




\section{Sensitivity to External Parameters}

The IBJ characteristics, as well as the effect of horizontal grid spacing might depend on the background conditions. Therefore, we quantify in this section $L$ and $M$, as well as the effect of resolution on the surface fluxes for a certain range of external parameters which is typical for winter-time CAOs in high latitudes.

It is well-known that thermally induced breeze-like circulations strongly depend on surface temperature contrasts as well as on the strength of the background flow, which corresponds to the large scale forcing. Therefore, it is of major interest to study the sensitivity of results to the absolute value of the geostrophic wind speed $\left|\mathbf{V}_{\mathbf{g}}\right|$ and to the difference between the surface temperatures of open water and sea ice $\Delta \theta$. As shown in Section 6.3, there is also a large sensitivity of the IBJ characteristics to the angle $\alpha$ between the vector of the undisturbed geostrophic wind far away from the ice edge and the vector normal to the sea ice edge. We explain the effect of $\alpha$ on the IBJ strength qualitatively using equation 3. Results are furthermore analyzed for various values of the inversion height $z_{i, i c e}$ over the sea ice and for different MIZ widths.

We present in the following subsections results of experiments where $\left|\mathbf{V}_{\mathbf{g}}\right|$ is varied in a range from 5 to $14 \mathrm{~ms}^{-1}, \Delta \theta$ from 15 to $35 \mathrm{~K}, \alpha$ from -60 to +60 degrees, $z_{i, i c e}$ from 120 to $340 \mathrm{~m}$ and the width of the MIZ from $L_{M I Z}=0 \mathrm{~km}$ to $L_{M I Z}=180 \mathrm{~km}$. Positive values of $\alpha$ correspond here to geostrophic wind from north-east and negative values to wind from north-west.

We have furthermore tested the sensitivity to the Coriolis parameter. However, CAOs are occurring in both hemispheres in latitudes between 60 and 80 degrees. There, the

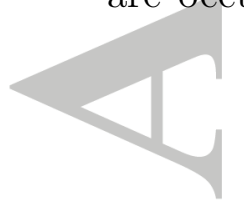


Coriolis parameter does not change much so that the sensitivity of the model results was also small.

We have also found, perhaps surprisingly, that $L$ and $M$, as well as the effect of grid spacing on the fluxes does not depend much on the stratification of the atmosphere above the inversion. Thus we do not show these results here.

\subsection{Sensitivity to the MIZ Width}

So far, we considered a narrow MIZ with a width $L_{M I Z}$ of about $10 \mathrm{~km}$. However, natural MIZs can have a larger width which would lead to a more gradual air mass transformation. This might in turn have an impact on the mesoscale structure of the simulated wind field. For this reason, we investigate also the effect of a smooth transition from high ice concentration to open water by prescribing the sea ice concentration $A$ according to equation (1). Several runs are carried out with various MIZ widths, where $L_{M I Z}$ is changing from $0 \mathrm{~km}$ to $180 \mathrm{~km}$.

First, we consider the results for $10 \mathrm{~m}$ height (Figure 10) as functions of the distance from the ice edge. We define the latter as the position where $A=0.95$. It can be seen that for larger $L_{M I Z}$ the ABL modification starts further downwind from the ice edge. This leads to very large differences between the simulated curves over the region extending from the ice edge to about $500 \mathrm{~km}$ downwind. This is in agreement with Pagowski and Moore [2001] who found that heat fluxes and air temperature during a CAO are strongly modified when a MIZ with an observed width was accounted for in their model. Also Liu et al. [2006] found a large impact of sea-ice concentration across the MIZ on the development of the boundary layer during a CAO in a region of about $300 \mathrm{~km}$ downstream from the ice

edge. Our results agree with Liu et al. who note that the effect of sea-ice concentration 
diminishes further downstream, suggesting that the atmosphere has a limited memory of the MIZ characteristics.

Figure 10 also shows, that for a larger value of $L_{M I Z}$ the position of the wind speed maximum is shifted further downwind from the ice edge, which also has an impact on the momentum flux. However, the absolute values of the wind speed maximum do not depend on $L_{M I Z}$. Moreover, Figure 10 suggests that the major difference between all the simulated curves is related simply with a parallel shift of all curves along the north-south axis. It can be seen that the magnitude of such a shift is approximately proportional to $L_{M I Z}$. A closer examination of Figure 10 suggests to plot the curves of corresponding variables obtained from runs with different $L_{M I Z}$ as functions of a new variable $y^{\prime}=y-y_{c}$, where $y^{\prime}$ is the distance from the center of the MIZ $y_{c}$.

In Figure 11, results are presented as functions of $y^{\prime}$. It can now be seen that, apart from the region close to the MIZ center, wind, temperature, and momentum fluxes of all runs agree well with each other downstream of $y_{c}$. Thus the MIZ width has only a small impact on the IBJ characteristics $M, L$ and $l$. This finding is important for the present study and the results shown later, which always assume a narrow MIZ. Only the scatter in the heat fluxes is large, but differences between runs occur in a narrow region of approximately $\pm 0.5 L_{M I Z}$ around the center of the MIZ. Especially, the results of the runs for $L_{M I Z}=0 \mathrm{~km}$ and $L_{M I Z}=45 \mathrm{~km}$ are close together where the latter fits well to often observed values of the MIZ width. This shows that the results based on the model runs with rapid transition are reliable in the region of at least $y^{\prime} \geq 0.5 L_{M I Z} \approx 20 \mathrm{~km}$, when compared to runs where the MIZ is well resolved.

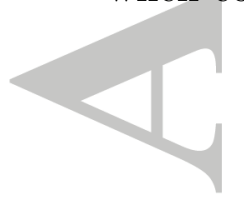


Another interesting result is obvious from Figure 11. It shows that the horizontal offset between the maxima of heat fluxes and wind speed depend on the value of $L_{M I Z}$. With increasing $L_{M I Z}$ the offset is decreasing.

The runs with different $L_{M I Z}$ are also repeated using $\Delta y=60 \mathrm{~km}$. The values of $A$ for the coarse-resolution grid are obtained by averaging the $A$ values used in the high resolution grid. The center of the MIZ $y_{c}$ (see equation (1)) is located exactly at the interface of two grid cells of the coarse-resolution grid.

Figure 12 shows absolute values of differences between high- and coarse-resolution runs related to the surface fluxes of heat and momentum. It is important to note that absolute values are chosen here instead of relative values because in the reference run the fluxes over sea ice are close to zero so that large relative values could be misinterpreted. It can be seen that both heat and momentum fluxes are underestimated between the ice edge and $200 \mathrm{~km}$ downstream by the coarse-resolution run in the whole range of considered values for $L_{M I Z}$. Close to the ice edge, the differences of surface fluxes of heat and momentum between the high- and coarse-resolution runs are found to decrease with increasing $L_{M I Z}$ but they never disappear. This holds, even for the large value $L_{M I Z}=180 \mathrm{~km}$. Moreover, for the value $L_{M I Z}=45 \mathrm{~km}$, which is typical in the north-western Fram Strait region [Hartmann et al., 1997], the effect of grid spacing on fluxes is nearly as large as for $L_{M I Z}=0 \mathrm{~km}$, so that we consider in the following sensitivity studies, only model runs with an abrupt change of ice to water for simplicity.

\subsection{Sensitivity to $\left|\mathrm{V}_{\mathrm{g}}\right|$}

Panels $a 1$ and $a 2$ of Figure 13 show that there is a very pronounced dependency of $L$ and $M$ on $\left|\mathbf{V}_{\mathbf{g}}\right| . L$ is almost linearly growing with increasing $\left|\mathbf{V}_{\mathbf{g}}\right|$, from about $80 \mathrm{~km}$ for $\left|\mathbf{V}_{\mathbf{g}}\right|$ 
$=5 \mathrm{~ms}^{-1}$ to about $290 \mathrm{~km}$ for $\left|\mathbf{V}_{\mathbf{g}}\right|=14 \mathrm{~ms}^{-1}$. The distance $l$ of the wind speed maximum from the ice edge depends also almost linearly on $\left|\mathbf{V}_{\mathbf{g}}\right|$ and is roughly proportional to $L$. $M$ is decreasing with increasing $\left|\mathbf{V}_{\mathbf{g}}\right|$, from about 0.6 for $\left|\mathbf{V}_{\mathbf{g}}\right|=5 \mathrm{~ms}^{-1}$ to less than 0.1 for $\left|\mathbf{V}_{\mathbf{g}}\right|=14 \mathrm{~ms}^{-1}$. This means that when $\left|\mathbf{V}_{\mathbf{g}}\right|$ is decreasing the resolution has to be increased to fulfill the resolution criterion given by equation (5). In agreement with this, the underestimation of $M$ is larger for lower $\left|\mathbf{V}_{\mathbf{g}}\right|$ (panel a2 of 13).

We find furthermore that the underestimation of both heat and momentum fluxes is larger in the coarse-resolution run when $\left|\mathbf{V}_{\mathbf{g}}\right|$ is decreasing (panels $a 1$ and a2 of Figure 14). This agrees well with the above findings based on the behavior of $L$ and $M$. The effect is most pronounced in the region between the ice edge and $120 \mathrm{~km}$ downstream. The maximum underestimation of momentum fluxes amounts to about $25 \%$ and occurs for $\left|\mathbf{V}_{\mathbf{g}}\right|=5 \mathrm{~ms}^{-1}$ over the first $60 \mathrm{~km}$ of open water. For $\left|\mathbf{V}_{\mathbf{g}}\right|=14 \mathrm{~ms}^{-1}$ the resolution effect on the momentum flux almost vanishes with only about $3 \%$ underestimation.

The effect of resolution on the heat flux is present in the whole range of $\left|\mathbf{V}_{\mathbf{g}}\right|$ values and is largest close to the ice edge. The underestimation of heat flux is largest for $\left|\mathbf{V}_{\mathbf{g}}\right|=$ $8 \mathrm{~ms}^{-1}$ and amounts up to $19 \%$ over the first $60 \mathrm{~km}$ of open water. The largest absolute value of the heat flux underestimation is about $80 \mathrm{Wm}^{-2}$ for $\left|\mathbf{V}_{\mathbf{g}}\right|=8 \mathrm{~ms}^{-1}$. Further downstream, the effect of grid spacing on heat flux is decreasing with an underestimation smaller than $5 \%$ at distances larger than $200 \mathrm{~km}$ from the ice edge. For $\left|\mathbf{V}_{\mathbf{g}}\right|=5 \mathrm{~ms}^{-1}$, there is even an overestimation of heat fluxes by the coarse-resolution run far from the ice edge but absolute values are small.

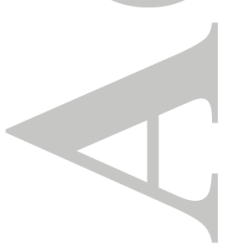




\subsection{Sensitivity to $\alpha$}

Figure 15 shows a remarkable sensitivity of the model results to the wind direction $\alpha$. This behaviour can be expected from the discussion in Section 4.1. Wind speed at the lowest model level as well as surface fluxes of heat and momentum vary strongly when different values of $\alpha$ are prescribed. A gradual change in the direction of the large-scale geostrophic wind from northeast to northwest results in a monotonous increase of the surface wind speed over open water from about 8 to $13 \mathrm{~ms}^{-1}$ in a $200 \mathrm{~km}$ region adjacent to the ice edge. When $\alpha$ is positive and increasing, the IBJ intensity is also increasing and its horizontal scale becomes smaller. For negative $\alpha$ and the IBJ disappears. The highest surface wind speeds of $13 \mathrm{~ms}^{-1}$ occur at a distance of about $100 \mathrm{~km}$ from the ice edge and are obtained for $\alpha=60^{\circ}$ (north-easterly wind). The lowest surface wind speeds of about $8 \mathrm{~ms}^{-1}$ occur over the $200 \mathrm{~km}$-wide region downstream of the ice edge. They are obtained by a run with northwest geostrophic wind $\left(\alpha=-60^{\circ}\right)$. For this wind direction, there is even a minimum in the surface wind speed which appears on the upstream side of the ice edge.

There is also a tremendous effect of $\alpha$ on the turbulent fluxes over open water as found for the wind maximum. The maximum surface momentum flux differs between 0.15 and $0.43 \mathrm{kgm}^{-1} \mathrm{~s}^{-2}$ dependent on $\alpha$, and the maximum heat flux varies between 380 and 680 $\mathrm{Wm}^{-2}$.

Values of $\left|\mathbf{G}_{\mathbf{m}}\right|$ can be estimated from equation (3) by using input values from the reference run at $200 \mathrm{~km}$ downstream the ice edge. Results for different values of $\alpha$ are presented in Figure 16 together with the corresponding ABL-mean wind speed obtained

from the NH3D simulations. It can be seen that the estimated values reproduce the 
modelled dependency of the ABL-mean wind speed on $\alpha$ well. Our estimates of $\left|\mathbf{G}_{\mathbf{m}}\right|$ differ from the ABL mean wind speed by less than $1.5 \mathrm{~ms}^{-1}$.

In section 4, it was shown that the asymmetry in the wind direction caused by the baroclinicity is partly cancelled out by Ekman turning. Considering Figure 15, this is confirmed by the numerical results showing that the wind direction in the convective ABL approaches $\alpha$. This holds, however, only for distances $>200 \mathrm{~km}$ from the ice edge where the assumptions of horizontal homogeneity needed for the estimation of the amount of Ekman turning are best fulfilled.

The effect of resolution on the surface fluxes is also sensitive to $\alpha$ (panels $b 1$ and $b 2$ of Figure 14). For coarse resolution, the underestimation of the momentum flux is largest when $\alpha=60^{\circ}$. It amounts to $15-20 \%$ in the region between the ice edge and about $150 \mathrm{~km}$ downstream. When $\alpha=-33.4^{\circ}$, the effect of resolution on the momentum flux vanishes. For $\alpha=-60^{\circ}$ the momentum flux is overestimated by the coarse-resolution run. Obviously, the resolution effect on the momentum flux is related to the underestimation of the IBJ intensity over open water.

The effect of resolution on the heat flux is largest for $\alpha=0^{\circ}$ with almost $20 \%$ flux underestimation over the first grid cell of the coarse-resolution run with open water. Interestingly, for $\alpha=-60^{\circ}$ the heat flux is underestimated over open water even further downstream from the ice edge and this underestimation remains approximately constant at $10 \%$ between $150 \mathrm{~km}$ and $280 \mathrm{~km}$ distance from the ice edge.

\subsection{Sensitivity to $\Delta \theta$}

The sensitivity of results to $\Delta \theta$ is described here for $\left|\mathbf{V}_{\mathbf{g}}\right|=8 \mathrm{~ms}^{-1}$. Panels $b 1$ and b2 of Figure 13 show that the IBJ characteristics depend strongly on $\Delta \theta$. The IBJ strength 
$M$ is increasing from about 0.09 to 0.55 when $\Delta \theta$ is changing from 15 to $35 \mathrm{~K}$. This shows that the IBJ can be clearly identified only for $\Delta \theta>15 \mathrm{~K}$. Panel b2 of Figure 13 also shows that an underestimation of $M$ by coarse-resolution is increasing with increasing $\Delta \theta$. The largest underestimation of $M$ occurs for $\Delta \theta=35 \mathrm{~K}$ and amounts to about $36 \%$.

When $\Delta \theta$ is varied from 15 to $35 \mathrm{~K}$, the characteristic length scale $L$ is decreasing from about 180 to $120 \mathrm{~km}$. This means that the resolution criterion (5) is never fulfilled in this

range of parameters. The momentum flux is underestimated by the coarse-resolution run between ice edge and $180 \mathrm{~km}$ downstream for the whole range of $\Delta \theta$ (Panel c2 of Figure 14). The effect vanishes further downstream. The most pronounced sensitivity to $\Delta \theta$ with respect to the effect of resolution is obtained at $90 \mathrm{~km}$ distance from the ice edge, which is exactly the position with the maximum wind speed. The largest underestimation of about $23 \%$ occurs for $\Delta \theta=35 \mathrm{~K}$, and the smallest one of about $12 \%$ at $\Delta \theta=15 \mathrm{~K}$.

The relative underestimation of heat flux depends only slightly on $\Delta \theta$. However, the absolute values of the differences between the heat fluxes increase with increasing $\Delta \theta$ (not shown). They change from $23 \mathrm{Wm}^{-2}$ close to the ice edge for $\Delta \theta=15 \mathrm{~K}$ to $91 \mathrm{Wm}^{-2}$ for $\Delta \theta=35 \mathrm{~K}$

Since for $\left|V_{g}\right|=8 \mathrm{~ms}^{-1}$ the resolution criteria (5) is not fulfilled for the whole range of the considered $\Delta \theta$, the effect of grid spacing on the surface fluxes never vanishes, although it decreases with decreasing $\Delta \theta$.

\subsection{Sensitivity to the Mixed Layer Height over Sea Ice}

Panel $c 2$ of Figure 13 demonstrates that $M$ also depends on the mixed layer height over ice $z_{i, i c e}$. Results are shown for $\left|V_{g}\right|=8 \mathrm{~ms}^{-1}$; they are similar for other wind speeds. $M$ is increasing from 0.35 to 0.5 when $z_{i, i c e}$ is changing from 120 to $340 \mathrm{~m}$. The effect 
of various $z_{i, i c e}$ is strong close to the ice edge but starts to vanish already at $120 \mathrm{~km}$ downstream from the ice edge (not shown here). the ice edge and the first $120 \mathrm{~km}$ over open water when $z_{i, i c e}$ is increasing (not shown here). In the coarse-resolution runs $M$ is underestimated by more than $30 \%$ and the underestimation is largest for $z_{i, i c e}=340 \mathrm{~m}$.

The characteristic length scales $L$ and $l$ (panel $c 1$ of Figure 13) show the tendency to decrease with increasing $z_{i, i c e}$ from values of $140 \mathrm{~km}$ to $120 \mathrm{~km}$ and from $90 \mathrm{~km}$ to $70 \mathrm{~km}$, respectively.

Surface fluxes of heat and momentum also increase with increasing $z_{i, i c e}$ (Figure 17). When $z_{i, i c e}$ increases from 120 to $340 \mathrm{~m}$, the maximum surface fluxes of heat increase from 600 to $750 \mathrm{Wm}^{-2}$, while momentum fluxes increase from 0.24 to $0.3 \mathrm{kgm}^{-1} \mathrm{~s}^{-2}$.

Figure 18 shows that the underestimation of both momentum and heat flux by the coarse-resolution run is almost not sensitive to the value of $z_{i, i c e}$. This is probably due to that horizontal scale $L$ is also not much sensitive to $z_{i, i c e}$.

\section{Convergence of Results with Decreasing $\Delta y$}

Here we consider the case with $\left|\mathbf{V}_{\mathbf{g}}\right|=8 \mathrm{~ms}^{-1}$ and $\alpha=33.4^{\circ}$ when the IBJ is well pronounced. Horizontal grid spacing is gradually decreased from $\Delta y=60 \mathrm{~km}$ till $\Delta y=$ $2.5 \mathrm{~km}$. The results for absolute wind speed at $10 \mathrm{~m}$ height are presented on panel $a$ of Figure 19. It can be seen that with $\Delta y=20 \mathrm{~km}$ the results are already very close to the run with $\Delta y=5 \mathrm{~km}$. The underestimation of maximum wind speed within the ABL with $\Delta y=20 \mathrm{~km}$ becomes less than $3 \%$, while the magnitude $M$ of the IBJ becomes

within $10 \%$ error from its value at $\Delta y=5 \mathrm{~km}$ (panel $b$ of Fig. 19). For the considered case characteristic length scale of the IBJ $L=140 \mathrm{~km}$. This suggests that the grid spacing 
necessary to resolve the IBJ with the accuracy of $10 \%$ with respect to its magnitude $M$ might be of about $L / 7$.

\section{Discussion}

The previous sections have shown several important results. The first one is the large sensitivity of the IBJ characteristics to the external forcing in both high and coarseresolution runs. Figure 13 shows that the IBJ width $L$ is most sensitive to the absolute value of the large-scale geostrophic wind speed $\left|\mathbf{V}_{\mathbf{g}}\right|$. Moreover, it is found that $L$ depends strongly on the geostrophic wind speed direction $\alpha$ relative to the ice edge orientation as ean be seen from Figure 15. Another characteristic of the IBJ, its strength $M$, is found to be strongly dependent on $\left|\mathbf{V}_{\mathbf{g}}\right|$ and $\alpha$, but, unlike $L$, it also has a large sensitivity to the difference $\Delta \Theta$ between the surface temperatures of sea ice and open water.

Our results show that the IBJ characteristics depend asymmetrically on the angle $\alpha$. The strongest IBJ and the largest heat and momentum fluxes occur for northeast directions of $\mathbf{V}_{\mathbf{g}}$ when the ice edge is oriented in east-west direction as in our study. Such wind directions prevail during the cold season over the MIZ of the Fram Strait and Greenland Sea [Gorshkov, 1983; Kolstad, 2008].

We have shown that such an asymmetric sensitivity of the results to $\alpha$ can be explained qualitatively by the effect of baroclinicity developing as a consequence of the intensive heating of the air mass advected over the open ocean during CAOs and by the effect of Coriolis force in this baroclinic ABL. This confirms that such baroclinicity is the main physical mechanism of the IBJ formation, as was also previously proposed by others [e.g. Reynolds, 1994; Bechtold et al., 1991; Brümmer, 1996]. The important role of such thermally-induced baroclinicity for the energy exchange between atmosphere and ocean 
during CAOs is demonstrated in Figure 15. Namely, over a large area of open water surface fluxes of heat and momentum can increase or decrease significantly depending on the angle $\alpha$. Our results also show that the strength of such effect depends on the season with a maximum sensitivity during winter, when heat fluxes and horizontal temperature gradients are largest.

The most pronounced dependences of the IBJ characteristics on the external forcing can be summarized in Figure 20 showing $L$ and $M$ as functions of $\left|\mathbf{V}_{\mathbf{g}}\right|$ and $\Delta \Theta$ (panels $a 1$ and $a 2$ of Figure 20) and of $\left|\mathbf{V}_{\mathbf{g}}\right|$ and $\alpha$ (panels $b 1$ and b2 of Figure 20). Figure 20 shows that the IBJ does not occur for a certain range of parameters, namely, there is no IBJ for large $\left|\mathbf{V}_{\mathbf{g}}\right|$, negative $\alpha$ and small $\Delta \Theta$.

In Figure 20, we also include values for $\left|\mathbf{V}_{\mathbf{g}}\right|, \alpha$, and $\Delta \Theta$ from previous CAO studies. Of particular interest are the observations of the 10 CAOs described by Brümmer $[1996$, 1999]. Despite some scatter, it is clearly seen that in his observations an IBJ occurred in the same range of parameters, or close to it, where our model results are also producing an IBJ. Moreover, cases with no observed IBJ correspond to our results with no IBJ occurrence as well. Figure 20 shows also that the observed external forcing during the CAO investigated by Wacker et al [2005] would not produce an IBJ, which is in agreement with their model results. On the contrary, in a case studied by Bechtold et al [1992], the external forcing is in a parameter range where an IBJ should exist (Figure 20). And indeed, the IBJ was produced by their model (their Figure 4). However, wind observations of this case are not available.

In flow regimes with convective rolls, one can expect a large spatial and temporal variability of wind, which might mask the appearence of the IBJ. Therefore, it is particularly 
important that the IBJ found in the mean fields of our mesoscale model is also found in the above mentioned spatially averaged observations of Brümmer. This supports our view explained in Section 2 that a mesoscale model is able to produce a realistic mean CAO structure.

Our estimates of the IBJ characteristics $L$ and $M$ might be relevant not only for marine CAOs but also for other cases with a CBL development. Similar atmospheric regimes are also observed during the cold seasons, for example, over the Great Lakes [Chang and Braham, 1991; Maesaka et al., 2006], over large polynyas in polar regions [Dare and Atkinson, 2000; Renfrew and King, 2000] and also over inland gulfs and seas such as, for example, the Baltic Sea [Vihma and Brümmer, 2002] and the Gulf of Finland [Savijarvi 2012]. It can be expected that over such regions wind and surface fluxes of heat and momentum show a similar dependence on external parameters as in the present study. This is especially important for polynyas where heat fluxes are also large [Kurtz and Bromwich, 1985; Kottmeier and Engelbart, 1992].

We have shown that a reproduction of the IBJ magnitude with $10 \%$ accuracy requires a grid spacing of about $L / 7$, which is about $15-20 \mathrm{~km}$. And indeed, we have found that the IBJ strength is underestimated in the coarse-resolution runs by up to $50 \%$ for some conditions. The value of this underestimation depends on the external forcing and according to Figure 13, the sensitivity of the IBJ strength $M$ to the external forcing is reduced when a model uses coarse resolution. Even though our results are based on idealized experiments they give a strong hint that the IBJ strength is not well reproduced in coarse-resolution climate models or reanalyses. For example, Kolstad [2008] has shown that the wind speed maximum along the ice edges in the Fram Strait and Greenland Sea 
regions found in winter months in QuikSCAT data (0.25 degree resolution) was absent in the NCEP/NCAR Reanalysis data (1.9 degree resolution). Also Renfrew et al. [2009] and Haine et al. [2009] demonstrate that coarse-resolution reanalyses tend to underestimate wind speed close to the ice edge. On the other hand, it has been shown [Moore and Renfrew, 2002; Renfrew et al., 2002] that the surface layer wind speed as observed during a ship campaign was reproduced even by coarse-resolution reanalyses sufficiently well for several cases of CAOs. The latter, nevertheless, does not necessarily mean that close to the ice edge reanalyses perform well since ship observations have limited spatial coverage and, therefore, might not capture the whole wind speed variability close to the ice edge. Although the resolution of the reanalyses is increasing with growing computer powers, the frequently used ERA Interim is still using a 0.7 degree grid so that the IBJ might be underestimated in it.

We have shown that the IBJ is strong enough to affect surface fluxes of heat and momentum. The underestimation of the IBJ strength leads to an underestimation primarily of momentum fluxes but also of heat fluxes, although the location of the maximal fluxes remains the same. From Figure 14 it can be seen that the underestimation of momentum flux is most sensitive to $\alpha$ but depends also strongly on $\Delta \Theta$ and $\left|\mathbf{V}_{\mathbf{g}}\right|$. However, the underestimation of heat flux is less sensitive to the external forcing.

Although the region with an underestimation of heat fluxes is limited to the first 120$180 \mathrm{~km}$ over open water adjacent to the ice edge, its effect might be important for climate modeling, because in this region heat fluxes are largest during CAOs. Even on the seasonal average the largest heat losses of the Arctic Ocean occur over the small area of the Fram Strait, where the warm West Spitzbergen current reaches the sea ice edge. There, 
CAOs represent a typical meteorological regime contributing largely to the heat exchange between ocean and atmosphere. In this region, monthly mean absolute values of heat fluxes over water in this region are exceeding those over the sea ice of the central Arctic at least by a factor of at least 10-15 [Przybylak, 2003]. For example, the underestimation of heat flux close to the ice edge might have an impact on the mixing of the upper layers of the ocean and water mass modification in this area. The latter occurs, for example,

along the ice edge in the Greenland sea modifying the water mass of the East Greenland Current, which is an important part of the circulation in the Nordic Seas [Mauritzen, $1996]$.

The results also hint to a possible uncertainty in the surface momentum fluxes during CAOs as obtained from climate models and coarse-resolution reanalyses. This might affect a number of processes in the MIZ region that are driven by wind stress, such as convective upwelling and downwelling of water masses as well as the sea ice drift [Häkkinen, 1987;

Guest and Davidson, 1991]. Finally, a correct simulation of the ice edge position in coupled models, which is crucial for CAO regimes [Wacker et al., 2005], depends also on the accuracy of wind stress simulations. Also the MIZ width, which - as we have shown (Figure 10) - in turn influences the heat fluxes and other variables, depends strongly on a correct modeling of momentum transport. Also the representation of the MIZ width and the distribution of sea ice within the MIZ is connected with large uncertainty in models and reanalyses due to coarse model grids used (e.g., the ERA Interim reanalyses is using a 0.7 degree grid). Another reason for this uncertainty is the coarse resolution of the sea ice concentration data itself. For example, the Reynolds sea ice concentration [Reynolds, 2001] used in many reanalyses is available only on a $1^{\circ}$ grid, and the old NCEP/NCAR 
reanalysis uses binary sea ice concentration which means that sea ice concentration is either 1 or 0 in each grid cell. One might expect, therefore, that recent reanalyses using high resolution, such as CFSR or ASR (Arctic System Reanalysis) [Bromwich et al., 2010], might differ strongly from the older ones over the region close to the ice edge with respect to different $\mathrm{ABL}$ parameters, especially during the $\mathrm{CAO}$ events.

\section{Conclusions}

The non-hydrostatic model NH3D was used with different horizontal grid spacing $\Delta y$ to simulate CAOs over Fram Strait. It was shown by comparing model results with each other and with observations that the typical mesoscale structures occuring during such CAOs can be sufficiently resolved using $\Delta y=5 \mathrm{~km}$ while a further increase of resolution to $\Delta y=1.25 \mathrm{~km}$ modified the results only marginally.

Our study showed that one of the characteristic features during CAOs consists in a wind speed maximum over open water. This maximum called ice-breeze jet (IBJ) in the literature, is simulated by NH3D at a distance of about 120-150 km downstream from the

ice edge. In a series of idealized runs the magnitude and horizontal width of the IBJ were quantified for a wide range of external parameters being typical for winter-time CAOs in high-latitudes. It was found that the IBJ characteristics are strongly sensitive to the external forcing. The strongest IBJ occurs for large temperature differences $\Delta \Theta$ between open water and sea ice and moderate large-scale geostrophic wind speed $\left|\mathbf{V}_{\mathbf{g}}\right|<10 \mathrm{~ms}^{-1}$ from northeast (when the ice edge is east-west oriented). The IBJ is only weak or does not exist at all for large $\left|\mathbf{V}_{\mathbf{g}}\right|$ from northwest and for weak $\Delta \Theta$. These results are in agreement with observations of several CAOs in the Arctic. 
We have shown that the ABL-averaged absolute wind speed and the ABL-averaged geostrophic wind show a similar asymmetric dependency of $\alpha$. The latter is related with a thermally induced change of the geostrophic wind in the ABL which is produced by the heating of the advected air mass. Based on these results, we believe that the thermal wind effect plays an important role for the variability of $M$.

The MIZ width $L_{M I Z}$ has a large impact on the simulated fields and especially on the locations of the IBJ and of the maxima of heat and momentum fluxes relative to the position of the ice edge. The latter was defined as the location where the ice concentration $\mathrm{A}=0.95$. Namely, the IBJ and the maximum of heat flux are located further downstream from the ice edge when $L_{M I Z}$ increases.

Using $\Delta y=60 \mathrm{~km}$, as being typical for regional climate models, we have quantified the effect of coarse horizontal grid spacing on the model results for an observed CAO. Thereby, our focus was on the effect on surface fluxes of heat and momentum. It was shown that the IBJ strength is underestimated by the coarse-resolution run. Consequently, surface fluxes of heat and momentum are also underestimated, but the size and location of the effect are different. The underestimation of momentum fluxes amounts typically to $15 \%$ in a region of $120-180 \mathrm{~km}$ downstream from the ice edge, while the underestimation of heat fluxes (also $15 \%$ ) is restricted to a smaller region close to the ice edge.

These underestimations of the IBJ strength and surface fluxes by the coarse-resolution runs were found to depend on external forcing. The largest underestimation of the IBJ strength of about $40 \%$ and of momentum fluxes of about $25 \%$ occurs for moderate geostrophic wind $\left|\mathbf{V}_{\mathbf{g}}\right|<10 \mathrm{~ms}^{-1}$ from northeast and for large $\Delta \Theta$. The underestimation

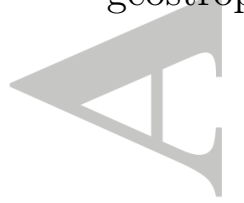


of surface heat flux was found to be less sensitive to external forcing with largest values of up to $20 \%$ close to the ice edge.

Since the areas of open water close to the MIZ belong to the regions with the largest heat loss of the Arctic Ocean, a $15 \%$ underestimation of heat fluxes in this region might have a significant impact on Arctic climate simulations. Thus one should keep in mind the possible uncertainty of the results of different coarse-resolution reanalyses or climate

models with respect to the simulated wind field and surface fluxes in the area close to the MIZ.

We did not consider the impact of vertical resolution here and restricted our investigation only to an idealized MIZ where, furthermore, small scale processes were not accounted for in detail. But we stress that all the results obtained are robust in the sense that they can be easily reproduced with other mesoscale models. In particular, we documented that the skill of the NH3D model was similar to that obtained with another nonhydrostatic model METRAS that was used earlier for the same CAO. Furthermore, we have shown that results of the two models agree remarkably well with each other with regard to all simulated fields despite differences in the numerical schemes. Most important for this agreement is the use of the same nonlocal turbulence closure in both models.

More detailed research is needed in the future to obtain a thorough understanding of the physical mechanisms influencing the IBJ. We stress once more that the present investigation represents an idealized study. A complete explanation of the IBJ mechanism would require in the future a more detailed study, for example, on the basis of new observations and convection resolving modelling with LES with full physics included and the use of the real sea ice distribution. Nevertheless, each method has its advantages and 
disadvantages, and a 3D modelling of convection covering the whole Fram Strait with the necessary grid size of about $100 \mathrm{~m}$ forms still a large challenge, especially when many runs are necessary to study the response on different external parameters. Thus, our results can be considered as an important step towards a better understanding of the ice-breeze regime since the results were obtained for a much wider range of external parameters than in previous studies.

Acknowledgments. We thank Victor Stepanenko, Andrey Glazunov, Vasiliy Lykosov, Jörg Hartmann, and Sergey Danilov for a discussion of many questions related with this study. The study was funded by the Russian Foundation for Basic Research (RFBR) (grant 11-05-00679-a), by Russian Ministry of Education and Science (contract 8833), Russian Government (grant No. 11.G34.31.0078 "Research under supervision of the leading scientist"), by the German Academic Exchange Service (DAAD) (A/10/84735) and by DFG (grant LU 818/3-1) and in some parts by the German Federal Ministry of Education and Research (project MiKlip, FKZ: 01LP1126A). The simulations with NH3D were performed on the supercomputer SKIF MSU "Chebyshev" at the Moscow University Supercomputing Center.

\section{Appendix A}

In the following, we derive an expression for the ratio between the absolute value of the total geostrophic wind (large scale and mesoscale) in the convective boundary layer over the open ocean and the absolute value of the large scale, height constant geostrophic 
wind. The derivation follows previous work [e.g. Lavoie, 1972; Overland et al., 1979] but is not exactly the same so that it is given in detail. We start with the expressions

$$
\begin{gathered}
u_{g m}=-\frac{1}{f z_{i}} \int_{0}^{z_{i}} \frac{1}{\rho} \frac{\partial p}{\partial y} d z \\
v_{g m}=\frac{1}{f z_{i}} \int_{0}^{z_{i}} \frac{1}{\rho} \frac{\partial p}{\partial x} d z
\end{gathered}
$$

where $u_{g m}$ and $v_{g m}$ are the components of the geostrophic wind averaged vertically over the convective $\mathrm{ABL}$, and $z_{i}$ is the mixed layer height. An analytical solution of the integrals becomes possible by using a zero-order jump mixed layer model for the profile of potential temperature. The latter was shown to be a good approximation in many earlier investigations [e.g. Lilly, 1968; Tennekes, 1973; Renfrew and King, 2000]. For $0<z<z_{i}$, we assume $\theta(z)=\theta_{m}$, where $\theta_{m}$ is the vertical average of the potential temperature in the ABL. The ABL capping inversion is assumed to be of infinitely small depth and is thus characterized by a discontinuous jump $\Delta \theta=\theta_{+}-\theta_{m}$ in potential temperature. The temperature profile above the inversion can be of any shape which satisfies hydrostatic balance. Using the ideal gas law and the definition of potential temperature, the hydrostatic equation can be written as

$$
\frac{\partial p^{k}}{\partial z}=-\frac{g p_{0}{ }^{k}}{c_{p} \theta}
$$

where $k=R / c_{p}$. Integrating (A2) from the undisturbed level $z=H$ where $\nabla \theta=0$ to some level $0<z<z_{i}$ gives

$$
p_{z}^{k}=p_{H}^{k}-\frac{g p_{0}{ }^{k}}{c_{p}} \int_{H}^{z} \theta^{-1} d z
$$

Since $\theta=\theta_{m}$ for $0<z<z_{i}$, we can rewrite (A3) as:

$$
p_{z}{ }^{k}=p_{H}{ }^{k}-\frac{g p_{0}{ }^{k}}{c_{p}}\left[\int_{H}^{z_{i}} \theta^{-1}+\frac{z-z_{i}}{\theta_{m}}\right] d z .
$$


Now, we take the horizontal gradient of (A4) and obtain after multiplication with $-\rho^{-1}=$ $-\theta_{m} R p^{k-1} / p_{0}{ }^{k}$

$$
-\rho^{-1} \nabla p_{z}=\frac{\theta_{m}}{\theta_{H}}\left(\rho^{-1} \nabla p\right)_{H}+g \theta_{m}\left[\nabla \int_{H}^{z_{i}} \theta^{-1} d z-\theta_{m}{ }^{-1} \nabla z_{i}-\frac{z-z_{i}}{\theta_{m}{ }^{2}} \nabla \theta_{m}\right]
$$

where $\nabla$ is the horizontal Nabla operator. The first term on the right-hand side of (A5) represents a forcing by the large scale pressure gradient and is thus equivalent to $f \mathbf{k} \times \mathbf{V}_{\mathbf{g}}$ where $\mathbf{V}_{\mathbf{g}}$ is the height constant large scale geostrophic wind.

We use the Leibniz rule to move $\nabla$ under the integral on the right-hand side of (A5) and after that average (A5) over the ABL depth

$$
\frac{1}{z_{i}} \int_{0}^{z_{i}}-\rho^{-1} \nabla p d z=f \mathbf{k} \times \mathbf{V}_{\mathbf{g}}-g \theta_{m} \int_{H}^{z_{i}} \theta^{-2} \nabla \theta d z-\frac{g \Delta \theta}{\theta_{+}} \nabla z_{i}+\frac{g z_{i}}{2 \theta_{m}} \nabla \theta_{m}
$$

An expression similar to (A6) was used in several studies [e.g. Lavoie, 1972; Keyser and Anthes, 1977; Overland et al., 1979] where, however, more approximations were used than for the above derivation.

The second term on the right-hand side (RHS) of (A6) is related to the horizontal gradients of potential temperature in the layer $z_{i}<z<H$. In our runs, these gradients originate from the vertical advection of temperature. Although this term is not small, estimates (not explained here in detail) show that the neglect of this term changes results only quantitatively $( \pm 5 \%$ in wind speed at distances over $200 \mathrm{~km}$ downstream from the ice edge), but the principal findings related to the thermal wind that we present in the present paper remain unchanged.

The third term on the RHS of (A6), containing the temperature jump $\Delta \theta$ at the inversion as defined above, represents the impact of the sloping inversion on the ABL-mean pressure gradient while the forth term arises from the ABL heating over water. These 
two terms are of opposite signs and might cancel each other, as argued by Fairall et al. [1987]. Therefore, we estimate here their magnitudes in the range of parameters that we consider in our study. To estimate first $\Delta \theta$, we use a simple closure (see, for example, Garrat, [1992], Chapter 6). The latter relates $\Delta \theta$ with the entrainment rate $\beta$ and follows as $\Delta \theta_{1}=\gamma_{\theta} \beta z_{i} /(1+2 \beta)$, where $\gamma_{\theta}$ is the potential temperature lapse rate above $z_{i}$. $\beta$ is thereby defined as the ratio between the heat fluxes at $z_{i}$ and at the surface so that $\beta=-\left(\overline{w^{\prime} \theta^{\prime}}\right)_{z_{i}} /\left(\overline{w^{\prime} \theta^{\prime}}\right)_{\text {surf }}$. The value of $\beta$ is commonly taken to be about 0.2 . We use furthermore $\theta_{m}=255 \mathrm{~K}, \partial \theta_{m} / \partial y=1 \times 10^{-4} \mathrm{Km}^{-1}, \gamma_{\theta}=0.01 \mathrm{Km}^{-1}, z_{i}=300 \mathrm{~m}$, $\partial z_{i} / \partial y=4.5 \times 10^{-3}$ which result from averages over the first $100 \mathrm{~km}$ downstream of the ice edge from our reference run. This all results finally in the values $-7.4 \times 10^{-5} \mathrm{~ms}^{-2}$ and $5.8 \times 10^{-4} \mathrm{~ms}^{-2}$ for the third and the fourth terms in (A6), respectively. Thus we can neglect the third term in our analysis as it is about an order of magnitude smaller than the forth one. This is confirmed also by figures (1) and (3) showing that $\Delta \theta$ is small.

Finally, after using (A6) in (A1), applying the simplifications mentioned in the previous paragraph, and keeping in mind that we consider a $2 \mathrm{D}$ problem so that $\partial p / \partial x=0$, we obtain the ABL-averaged components of the geostrophic wind $\mathbf{G}_{\mathbf{m}}$ as

$$
\begin{aligned}
& u_{g m}=U_{g}+\frac{g z_{i}}{2 f \theta_{m}} \frac{\partial \theta_{m}}{\partial y}, \\
& v_{g m}=V_{g}
\end{aligned}
$$

where $U_{g}$ and $V_{g}$ are the components of the large scale, height constant geostrophic wind with $U_{g}=\left|\mathbf{V}_{\mathbf{g}}\right| \sin \alpha$ and $V_{g}=\left|\mathbf{V}_{\mathbf{g}}\right| \cos \alpha$ and $\alpha$ is the angle between the direction of the large-scale geostrophic wind $\mathbf{V}_{\mathbf{g}}$ and the direction orthogonal to the ice edge. We define the thermally induced geostrophic wind averaged over the ABL depth as $\mathbf{V}_{\mathbf{g t}}=\mathbf{G}_{\mathbf{m}}-\mathbf{V}_{\mathbf{g}}$, so that according to $(\mathrm{A} 7) \mathbf{V}_{\mathbf{g t}}=g z_{i} /\left(2 f \theta_{m}\right) \partial \theta_{m} / \partial y \cdot \mathbf{i}$, where $\mathbf{i}$ is the unit vector in westward 
direction. Thus we can write for the magnitude $\left|\mathbf{G}_{\mathbf{m}}\right|$ of the ABL-averaged geostrophic wind

$$
\left|\mathbf{G}_{\mathbf{m}}\right|=\left[\left(\left|\mathbf{V}_{\mathbf{g}}\right| \sin \alpha+\mathbf{V}_{\mathbf{g t}} \cdot \mathbf{i}\right)^{2}+\left(\left|\mathbf{V}_{\mathbf{g}}\right| \cos \alpha\right)^{2}\right]^{1 / 2}
$$

Division of (A8) by the absolute value of the large-scale geostrophic wind $\left|\mathbf{V}_{\mathbf{g}}\right| \neq 0$ gives

$$
\frac{\left|\mathbf{G}_{\mathbf{m}}\right|}{\left|\mathbf{V}_{\mathbf{g}}\right|}=\left[1+2 \frac{\mathbf{V}_{\mathbf{g t}} \cdot \mathbf{i}}{\left|\mathbf{V}_{\mathbf{g}}\right|} \sin \alpha+\left(\frac{\mathbf{V}_{\mathbf{g t}} \cdot \mathbf{i}}{\left|\mathbf{V}_{\mathbf{g}}\right|}\right)^{2}\right]^{1 / 2} \text {. }
$$

It is worth to note that if we define the averaged over the ABL depth thermal wind $\mathbf{V}_{\mathbf{T}}$ as a vector difference between $\mathbf{V}_{\mathbf{g}}$ above the $\mathrm{ABL}$ and $\mathbf{G}_{\mathbf{m}}$, then $\mathbf{V}_{\mathbf{T}}=-\mathbf{V}_{\mathbf{g t}}$. With $y$-axis directed from north to south and $x$-axis from east to west (see Figure 6 ) $\partial \theta_{m} / \partial y>0$ and

$\mathbf{V}_{\mathbf{g t}}$ is directed from east to west, leading to westward deflection of $\mathbf{G}_{\mathbf{m}}$. This corresponds to $\mathbf{V}_{\mathbf{T}}$ directed from west to east.

\section{Appendix B}

We simulate the NH3D reference case also with the nonhydrostatic model METRAS.

The main purpose is to estimate the relative importance of model specific approximations and of the numerical frameworks for the obtained results. This also stresses the effect of turbulence parameterizations which are the same in both models. The close agreement of the results explained below brings more confidence to the main conclusions of the paper based on the NH3D results only.

The model METRAS has originally been developed at the Meteorological Institute of the University of Hamburg [Schlünzen, 1990]. It is based on the primitive equations in a nonhydrostatic and anelastic mode and is applied here in a 2D-version which was described in detail by Lüpkes and Schlünzen [1996]. The model has been successfully applied to 
estimate atmospheric trans-coastal fluxes of pollutants, to study tracer transports, and many times for the modeling of boundary layer processes in Arctic regions, e.g. during on-ice flow [Vihma et al., 2003; Vihma et al., 2005] and off-ice flow over the open ocean [Lüpkes and Schlünzen, 1996] as well as over the marginal sea-ice zone [Birnbaum and Lüpkes, 2002]. Furthermore, it was used for the modeling of convection over leads in Arctic sea-ice [Lüpkes et al., 2008a; 2008b].

It is important that the turbulence closure used in NH3D repeats that of METRAS, namely the combination of a nonlocal in the convective ABL and a local closure elsewhere. Also, the parameterization of turbulent fluxes in the surface layer based on MoninObukhov similarity theory is the same in METRAS as in NH3D.

For a detailed model description, we refer to the references mentioned above and concentrate here only on the differences to the NH3D model. The most important one is the different coordinate system which is for METRAS also terrain following but z-dependent.

METRAS uses the anelastic approximation which results in an elliptic equation for the pressure perturbation. The latter is solved by a conjugate gradients method [Kapitza and Eppel, 1987] while the corresponding equation in NH3D is solved iteratively applying a direct method. Advection and diffusion of scalars are solved in METRAS forward in time and upstream in space, while advection of wind is calculated with the Adams-Bashforth scheme using centered differences in space. Unlike the forth-order diffusion in NH3D, a 7-point Shapiro filter, similar to sixth-order diffusion is used in horizontal direction in METRAS. It is applied every 10th time step. The lateral boundary conditions differ from those used in NH3D, especially, at the inflow boundary where, instead of fixed values as

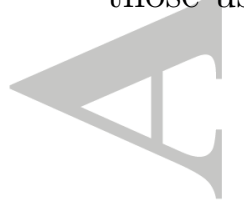


in NH3D, zero gradient conditions are used for both scalars and for the boundary-parallel wind component.

In Figure 21 we present the simulation results of the reference case by the METRAS model using $\delta y=5 \mathrm{~km}$. A comparison of figures 21 and 3 shows that absolute values and spatial patterns of the simulated fields are very similar in NH3D and METRAS. The most important for this study is a striking agreement with regards to the simulated absolute wind speed and its maximum values of about $13.6 \mathrm{~ms}^{-1}$ in the core of the IBJ. Also the values of turbulent fluxes of heat and momentum agree very well: heat fluxes in both models exceed $600 \mathrm{Wm}^{-2}$ and momentum fluxes have a maximum at the surface of about $0.35 \mathrm{kgm}^{-1} \mathrm{~s}^{-2}$.

Such a high degree of agreement between the two models with different numerical framework and model equations is only possible by using the same turbulence closures. The use of other closures has a very significant effect on simulated vertical profiles of potential temperature as shown already by Lüpkes and Schlünzen [1996]. In the latter the authors have shown a better skill of the nonlocal closures versus local closure to reproduce observations. This points to the large impact of the physical parameterizations.

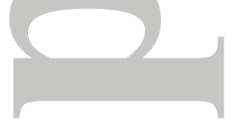

\section{References}

Andreas, E. L., T. W. Horst, A. A. Grachev, P. O. G. Persson, C. W. Fairall, P. S. Guest, and R. E. Jordan (2010), Parametrizing turbulent exchange over summer sea ice and the marginal ice zone, Quart. J. of the Roy. Meteor. Soc., 136(649), 927-943, doi:

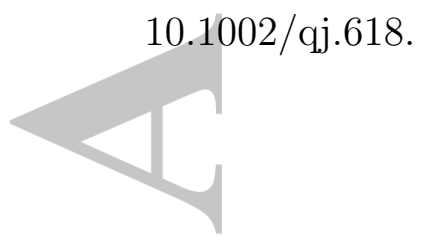


Anthes, R. A., N. L. Seaman, and T. T. Warner (1980), Comparisons of Numerical Simulations of the Planetary Boundary Layer by a Mixed-Layer and a Multi-Level Model, Mon. Wea. Rev., 108(3), 365-376, doi: 10.1175/1520-0493(1980)108<0365:consot>2.0.co;2.

Anthes, R. A., D. Keyser, and J. W. Deardorff (1982), Further Considerations on Modeling the Sea Breeze with a Mixed-Layer Model, Mon. Wea. Rev., 110(7), 757-765, doi: 10.1175/1520-0493(1982)110<0757:fcomts >2.0.co;2.

Atkinson, B. W., and J. Wu Zhang (1996), Mesoscale shallow convection in the atmosphere, Rev. Geophys., 34(4), 403-431, doi: 10.1029/96rg02623.

Arya, S. P. S., and J. C. Wyngaard (1975), Effect of Baroclinicity on Wind Profiles and the Geostrophic Drag Law for the Convective Planetary Boundary Layer, J. Atmos. Sci., 32(4), 767-778, doi: 10.1175/1520-0469(1975)032<0767:eobowp>2.0.co;2.

Ayotte, K. W., et al. (1996), An evaluation of neutral and convective planetary boundarylayer parameterizations relative to large eddy simulations, Boundary-Layer Meteorol., r9(1), 131-175, doi: 10.1007/bf00120078.

Bechtold, P., J.-P. Pinty, and F. Mascart (1991), A Numerical Investigation of the Influence of Large-Scale Winds on Sea-Breeze- and Inland-Breeze-type Circulations, J. Appl. Meteor., 30(9), 1268-1279, doi: 10.1175/1520-0450(1991)030<1268:anioti>2.0.co;2.

Bechtold, P., C. Fravalo, and J.-P. Pinty (1992), A study of a two-dimensional cloudiness transition during a cold air outbreak event, Boundary-Layer Meteorol., 60(3), 243-270, doi: $10.1007 / \mathrm{bf00119378.}$

Birnbaum, G., and C. Lüpkes (2002), A new parameterization of surface drag in the marginal sea ice zone, Tellus A, 54(1), 107-123, doi: 10.1034/j.1600-0870.2002.00243.x. 
Bromwich, D., Y.-H. Kuo, M. Serreze, J. Walsh, L.-S. Bai, M. Barlage, K. Hines, and A. Slater (2010), Arctic System Reanalysis: Call for Community Involvement, Eos, Transactions American Geophysical Union, 91(2), 13-14, doi: 10.1029/2010eo020001.

Brown, A. R. (1996), Large-eddy simulation and parametrization of the baroclinic boundary-layer, Quart. J. of the Roy. Meteor. Soc., 122(536), 1779-1798, doi: 10.1002/qj.49712253603.

Brümmer, B. (1996), Boundary-layer modification in wintertime cold-air outbreaks from the Arctic sea ice, Boundary-Layer Meteorol., 80(1), 109-125, doi: 10.1007/bf00119014.

Brümmer, B. (1997), Boundary Layer Mass, Water, and Heat Budgets in Wintertime Cold-Air Outbreaks from the Arctic Sea Ice, Mon. Wea. Rev., 125(8), 1824-1837, doi: 10.1175/1520-0493(1997)125<1824:blmwah>2.0.co;2.

Brümmer, B. (1999), Roll and Cell Convection in Wintertime Arctic ColdAir Outbreaks, J. Atmos. Sci., 56(15), 2613-2636, doi: 10.1175/15200469(1999)056<2613:racciw >2.0.co;2.

Byun, D.-W., and S. P. S. Arya (1986), A study of mixed-layer momentum evolution, Atmospheric Environment, 20(4), 715-728, doi: http://dx.doi.org/10.1016/00046981(86)90186-1.

Chang, S. S., and R. R. Braham (1991), Observational Study of a Convective Internal Boundary Layer over Lake Michigan, J. Atmos. Sci., 48(20), 2265-2279, doi: 10.1175/1520-0469(1991)048<2265:osoaci $>2.0$. co;2.

Chrobok, G., S. Raasch, and D. Etling (1992), A comparison of local and non-local turbulence closure methods for the case of a cold air outbreak, Boundary-Layer Meteorol., 58(1), 69-90, doi: 10.1007/bf00120752. 
Cotton, W. R., and R. A. Anthes (1989), Storm and cloud dynamics, 883 pp., Academic Press, Inc., San Diego, CA.

Dee, D. P. and Coauthors, (2011), The ERA-Interim reanlysis: Configuration and performance of the data assymilation system, Quart. J. of the Roy. Meteor. Soc., 137. 553-597.

Dyer, A. J. (1974), A review of flux-profile relationships, Boundary-Layer Meteorol., 7(3), 363-372, doi: 10.1007/bf00240838.

Etling, D., and R. A. Brown (1993), Roll vortices in the planetary boundary layer: A review, Boundary-Layer Meteorol., 65(3), 215-248, doi: 10.1007/bf00705527.

Fairall, C. W., and R. Markson (1987), Mesoscale Variations in Surface Stress, Heat Fluxes, and Drag Coefficient in the Marginal Ice Zone During the 1983 Marginal Ice Zone Experiment, J. Geophys. Res., 92(Cr), 6921-6932, doi: 10.1029/JC092iC07p06921.

Garratt, J. R. (1992), The Atmospheric Boundary Layer, 336 pp., Cambridge University Press, Cambridge.

Glendening, J. W. (1994), Dependence of boundary layer structure near an ice-edge coastal front upon geostrophic wind direction, J. Geophys. Res., 99(D3), 5569-5581, doi: $10.1029 / 93 \mathrm{jd} 02925$.

Guest, P. S., and K. L. Davidson (1991), Meteorological Triggers for Deep Convection in the Greenland Sea, in Elsevier Oceanography Series, edited by P. C. Chu and J. C. Gascard, pp. 369-375, Elsevier.

Guest, P. S., K. L. Davidson, J. E. Overland, and P. A. Frederickson (1995a), Atmosphereocean interactions in the marginal ice zones of the Nordic Seas, in Arctic Oceanography: Marginal Ice Zones and Continental Shelves, edited, pp. 51-95, AGU, Washington, DC. 
Guest, P. S., J. W. Glendening, and K. L. Davidson (1995b), An observational and numerical study of wind stress variations within marginal ice zones, J. Geophys. Res., 100(C6), 10887-10904, doi: 10.1029/94jc03391.

Gorshkov, S. G. (Ed.) (1983), World Ocean Atlas. Volume 3: Arctic Ocean, (in Russian), 189 pp., Pergamon Press, Oxford.

Gryanik, V. M., T. N. Doronina, D. J. Olbers, and T. H. Warncke (2000), The theory of three-dimensional hetons and vortex-dominated spreading in localized turbulent convection in a fast rotating stratified fluid, J. Fluid Mech., 423, 71-125, doi: doi:10.1017/S002211200000183X.

Gryanik, V. M., and J. Hartmann (2002), A Turbulence Closure for the Convective Boundary Layer Based on a Two-Scale Mass-Flux Approach, J. Atmos. Sci., 59(18), 27292744, doi: 10.1175/1520-0469(2002)059<2729:atcftc >2.0.co;2.

Gryanik, V. M., J. Hartmann, S. Raasch, and M. Schröter (2005), A Refinement of the Millionshchikov Quasi-Normality Hypothesis for Convective Boundary Layer Turbulence, J. Atmos. Sci., 62(7), 2632-2638, doi: 10.1175/jas3457.1.

Gryschka, M., C. Drüe, D. Etling, and S. Raasch (2008), On the influence of sea-ice inhomogeneities onto roll convection in cold-air outbreaks, Geophys. Res. Lett., 35(23), L23804, doi: 10.1029/2008gl035845.

Govind, P. K. (1975), Omega Windfinding Systems, J. Appl. Meteor., 14(8), 1503-1511, doi: 10.1175/1520-0450(1975)014<1503:ows >2.0.co;2.

Haine, T. W. N., S. Zhang, G. W. K. Moore, and I. A. Renfrew (2009), On the impact of high-resolution, high-frequency meteorological forcing on Denmark Strait ocean circulation, Quart. J. of the Roy. Meteor. Soc., 135(645), 2067-2085, doi: 10.1002/qj.505. 
Häkkinen, S. (1987), Upwelling At The Ice Edge: A Mechanism For Deep Water Formation?, J. Geophys. Res., 92(C5), 5031-5034, doi: 10.1029/JC092iC05p05031.

Harrington, J. Y., and P. Q. Olsson (2001), On the potential influence of ice nuclei on surface-forced marine stratocumulus cloud dynamics, J. Geophys. Res., 106(D21), 27473-27484, doi: 10.1029/2000jd000236.

Hartmann, J., C. Kottmeier, and S. Raasch (1997), Roll Vortices and Boundary-Layer Development during a Cold Air Outbreak, Boundary-Layer Meteorol., 84(1), 45-65, doi: 10.1023/a:1000392931768.

Hartmann, J., et al. (1999), Arctic Radiation and Turbulence Interaction Study (ARTIST) Reports on Polar Research, 81 pp, Alfred Wegener Institute for Polar and Marine Research, Bremerhaven

Holtslag, A. A. M., and C.-H. Moeng (1991), Eddy Diffusivity and Countergradient Transport in the Convective Atmospheric Boundary Layer, J. Atmos. Sci, 48(14), 1690-1698, doi: 10.1175/1520-0469(1991)048<1690:edacti $>2.0$. co;2.

Honnert, R., V. Masson, and F. Couvreux (2011), A Diagnostic for Evaluating the Representation of Turbulence in Atmospheric Models at the Kilometric Scale, J. Atmos. Sci., 68(12), 3112-3131, doi: 10.1175/jas-d-11-061.1.

Hundsdorfer, W., and R. A. Trompert (1994), Method of lines and direct discretization: a comparison for linear advection, Appl. Numer. Math., 13(6), 469-490, doi: 10.1016/0168-9274(94)90009-4.

Kapitza, H., and D. Eppel (1987), A 3-D poisson solver based on conjugate gradients compared to standard iterative methods and its performance on vector computers, $J$. Comput. Phys., 68(2), 474-484, doi: 10.1016/0021-9991(87)90067-2. 
Kattsov, V., and E. Källén (2005), Future climate change: Modeling and scenarios for the Arctic. In: Arctic Climate Impact Assessment (ACIA). Cambridge University Press, Cambridge, UK, pp. 99150.

Kolstad, E. W. (2008), A QuikSCAT climatology of ocean surface winds in the Nordic seas: Identification of features and comparison with the NCEP/NCAR reanalysis, $J$. Geophys. Res., 113(D11), D11106, doi: 10.1029/2007jd008918.

Kolstad, E., and T. Bracegirdle (2008), Marine cold-air outbreaks in the future: an assessment of IPCC AR4 model results for the Northern Hemisphere, Clim. Dynam., 30(7), 871-885, doi: 10.1007/s00382-007-0331-0.

Kottmeier, C., and D. Engelbart (1992), Generation and atmospheric heat exchange of coastal polynyas in the Weddell Sea, Boundary-Layer Meteorol., 60(3), 207-234, doi: $10.1007 / \mathrm{bf00119376.}$

Kottmeier, C., J. Hartmann, C. Wamser, A. Bochert, C. Lüpkes, D. Freese, and W. Cohrs (1994), Radiation and eddy flux experiment 1993 (REFLEX II), Reports on Polar Research, 133, AWI, Bremerhaven, 62 pp.

Kurtz, D. D., and D. H. Bromwich (1985), A recurring, atmospherically forced polynya in Terra Nova Bay, in Oceanology of the Antarctic Continental Shelf, Antarct. Res. Ser., vol. 43, edited by S. Jacobs, pp. 177201, AGU, Washington, D. C., doi:10.1029/AR043p0177.

Langland, R. H., P. M. Tag, and R. W. Fett (1989), An ice breeze mechanism for boundarylayer jets, Boundary-Layer Meteorol., 48(1), 177-195, doi: 10.1007/bf00121789.

Lavoie, R. L. (1972), A Mesoscale Numerical Model of Lake-Effect Storms, J. Atmos. Sci., 29(6), 1025-1040, doi: 10.1175/1520-0469(1972)029<1025:amnmol>2.0.co;2. 
Lilly, D. K. (1968), Models of cloud-topped mixed layers under a strong inversion, Quart. J. of the Roy. Meteor. Soc., 94(401), 292-309, doi: 10.1002/qj.49709440106.

Liu, A. Q., G. W. K. Moore, K. Tsuboki, and I. A. Renfrew (2004), A high-resolution simulation of convective roll clouds during a cold-air outbreak, Geophys. Res. Lett., 31(3), L03101, doi: 10.1029/2003gl018530.

Liu, A., G. Moore, K. Tsuboki, and I. Renfrew (2006), The Effect of the Sea-ice Zone on the Development of Boundary-layer Roll Clouds During Cold Air Outbreaks, BoundaryLayer Meteorol., 118(3), 557-581, doi: 10.1007/s10546-005-6434-4.

Lüpkes, C., and K. H. Schlünzen (1996), Modelling the arctic convective boundary-layer with different turbulence parameterizations, Boundary-Layer Meteorol., 79(1), 107-130, doi: $10.1007 / \mathrm{bf} 00120077$.

Lüpkes, C., and G. Birnbaum (2005), Surface drag in the Arctic marginal sea-ice zone: a comparison of different parameterisation concepts, Boundary Layer Meteorol., 117, 179-211, doi: 10.1007/s10546-005-1445-8.

Lüpkes, C., T. Vihma, G. Birnbaum, and U. Wacker (2008a), Influence of leads in sea ice on the temperature of the atmospheric boundary layer during polar night, Geophys. Res. Lett., 35(3), L03805, doi: 10.1029/2007gl032461.

Lüpkes, C., Gryanik, V., Witha, B., Gryschka, M., Raasch, S., and Gollnik, T. (2008b), Modeling convection over arctic leads with LES and a non-eddy-resolving microscale model, J. Geophys. Res., 113, C09028, doi:10.1029/2007JC004099.

Lüpkes, C., Vihma, T., Birnbaum, G., Dierer, S., Garbrecht, T., Gryanik, V., Gryschka, M., Hartmann, J., Heinemann, G., Kaleschke, L., Raasch, S., Savijärvi, H., Schlünzen, K., and Wacker, U. (2012), Mesoscale modelling of the Arctic atmospheric boundary 
layer and its interaction with sea ice, Chapter 7 in: ARCTIC climate change - The ACSYS decade and beyond (P. Lemke and H.-W. Jacobi (Edts.)) Springer, Atmospheric and Oceanographic Sciences Library, 43, doi:10.1007/978-94-007-2027-5.

Lock, A. P., A. R. Brown, M. R. Bush, G. M. Martin, and R. N. B. Smith (2000), A New Boundary Layer Mixing Scheme. Part I: Scheme Description and SingleColumn Model Tests, Mon. Wea. Rev., 128(9), 3187-3199, doi: 10.1175/15200493(2000)128<3187:anblms>2.0.co;2.

Maesaka, T., G. W. K. Moore, Q. Liu, and K. Tsuboki (2006), A simulation of a lake effect snowstorm with a cloud resolving numerical model, Geophys. Res. Lett., 33(20), L20813, doi: 10.1029/2006gl026638.

Mahrer, Y., and R. A. Pielke (1977), The Effects of Topography on Sea and Land Breezes in a Two-Dimensional Numerical Model, Mon. Wea. Rev., 105(9), 1151-1162, doi: 10.1175/1520-0493(1977)105<1151:teotos >2.0.co;2.

Mai, S., C. Wamser, and C. Kottmeier (1996), Geometric and aerodynamic roughness of sea ice, Boundary-Layer Meteorol., 7r(3), 233-248, doi: 10.1007/bf00123526.

Marshall, J., and F. Schott (1999), Open-ocean convection: Observations, theory, and models, Rev. Geophys., 37(1), 1-64, doi: 10.1029/98rg02739.

Mauritzen, C. (1996), Production of dense overflow waters feeding the North Atlantic across the Greenland-Scotland Ridge. Part 1: Evidence for a revised circulation scheme, Deep Sea Research Part I: Oceanographic Research Papers, 43(6), 769-806, doi: http://dx.doi.org/10.1016/0967-0637(96)00037-4.

Miranda, P. M. A., and I. N. James (1992), Non-Linear Three-Dimensional Effects On Gravity-Wave Drag: Splitting Flow and Breaking Waves, Quart. J. of the Roy. Meteor. 
Soc, 118(508), 1057-1081, doi: 10.1002/qj.49711850803.

Miranda, P. M. A., and M. A. Valente (1997), Critical Level Resonance in ThreeDimensional Flow past Isolated Mountains, J. Atmos. Sci., 54(12), 1574-1588, doi: 10.1175/1520-0469(1997)054<1574:CLRITD>2.0.CO;2.

Müller, G., and A. Chlond (1996), Three-dimensional numerical study of cell broadening during cold-air outbreaks, Boundary-Layer Meteorol., 81(3-4), 289-323, doi: $10.1007 / \mathrm{bf02430333.}$

Moore, G. W. K., and I. A. Renfrew (2002), An Assessment of the Surface Turbulent Heat Fluxes from the NCEPNCAR Reanalysis over the Western Boundary Currents, $J$ Climate, 15(15), 2020-2037, doi: 10.1175/1520-0442(2002)015<2020:aaotst>2.0.co;2.

Noh, Y., W. G. Cheon, S. Y. Hong, and S. Raasch (2003), Improvement of the Kprofile Model for the Planetary Boundary Layer based on Large Eddy Simulation Data, Boundary-Layer Meteorol., 107(2), 401-427, doi: 10.1023/a:1022146015946.

Orlanski, I. (1976), A simple boundary condition for unbounded hyperbolic flows, $J$. Comput. Phys., 21(3), 251-269, doi: 10.1016/0021-9991(76)90023-1.

Overland, J. E., R. M. Reynolds, and C. H. Pease (1983), A Model of the Atmospheric Boundary Layer Over the Marginal Ice Zone,J. Geophys. Res., 88(C5), 2836-2840, doi: 10.1029/JC088iC05p02836.

Pagowski, M., and G. W. K. Moore (2001), A Numerical Study of an Extreme Cold-Air Outbreak over the Labrador Sea: Sea Ice, Air-Sea Interaction, and Development of Polar Lows, Mon. Wea. Rev., 129(1), 47-72, doi:10.1175/15200493(2001)129<0047:ANSOAE > 2.0.CO;2. 
Przybylak, R. (2003), The Climate of the Arctic, 288 pp., Kluwer Academic Publishers, Dordrecht/Boston/London.

Raymond, W. H., and H. L. Kuo (1984), A radiation boundary condition for multidimensional flows, Quart. J. of the Roy. Meteor. Soc, 110(464), 535-551, doi: 10.1002/qj.49711046414.

Renfrew, I. A., and J. C. King (2000), A Simple Model Of The Convective Internal Boundary Layer And Its Application To Surface Heat Flux Estimates Within Polynyas, Boundary-Layer Meteorol., 94(3), 335-356, doi: 10.1023/a:1002492412097.

Renfrew, I. A., and G. W. K. Moore (1999), An Extreme Cold-Air Outbreak over the Labrador Sea: Roll Vortices and Air-Sea Interaction, Mon. Wea. Rev., 127(10), 23792394, doi: 10.1175/1520-0493(1999)127<2379:aecaoo>2.0.co;2.

Renfrew, I. A., G. W. K. Moore, P. S. Guest, and K. Bumke (2002), A Comparison of Surface Layer and Surface Turbulent Flux Observations over the Labrador Sea with ECMWF Analyses and NCEP Reanalyses, J. Phys. Oceanogr., 32(2), 383-400, doi: 10.1175/1520-0485(2002)032<0383:acosla $>2.0 . c 0 ; 2$.

Renfrew, I. A., G. N. Petersen, D. A. J. Sproson, G. W. K. Moore, H. Adiwidjaja, S. Zhang, and R. North (2009), A comparison of aircraft-based surface-layer observations over Denmark Strait and the Irminger Sea with meteorological analyses and QuikSCAT winds, Quart. J. of the Roy. Meteor. Soc, 135(645), 2046-2066, doi: 10.1002/qj.444.

Reynolds, M. (1984), On the Local Meteorology at the Marginal Ice Zone of the Bering Sea, J. Geophys. Res., 89(C4), 6515-6524, doi: 10.1029/JC089iC04p06515.

Rienecker, M. M. and Coauthors, (2011), MERRA: NASA's Modern-Era Retrospective Analysis for Research and Applications. J. Climate, 24, 3624-3648. 
Roach, A. T., K. Aagaard, and F. Carsey (1993), Coupled ice-ocean variability in the Greenland Sea, Atmosphere-Ocean, 31(3), 319-337, doi: 10.1080/07055900.1993.9649474.

Saha, S., et al. (2010), The NCEP Climate Forecast System Reanalysis, Bull. Amer. Meteor. Soc., 91(8), 1015-1057, doi: 10.1175/2010bams3001.1.

Savijärvi, H. I. (2012), Cold air outbreaks over high-latitude sea gulfs. Tellus A 2012, 64, doi: $10.3402 /$ tellusa.v64i0.12244.

Schlünzen, K. H. (1990), Numerical Studies on the Inland Penetration of Sea Breeze Fronts at a Coastline with Tidally Flooded Mudflats, Beitr. Physik Atm., 63, 254-256.

Schott, F., M. Visbeck, and J. Fischer (1993), Observations of Vertical Currents and Convection in the Central Greenland Sea During the Winter of 1988-1989, J. Geophys. Res., 98(C8), 14401-14421, doi: 10.1029/93jc00658.

Schröter, M., S. Raasch, and H. Jansen (2005), Cell Broadening Revisited: Results from High-Resolution Large-Eddy Simulations of Cold Air Outbreaks, J. Atmos. Sci., 62(6), 2023-2032, doi: 10.1175/jas3451.1.

Sorbjan, Z. (2004), Large-eddy simulations of the baroclinic mixed layer, Boundary-Layer Meteorol., 112(1), 57-80, doi: 10.1023/B:BOUN.0000020161.99887.b3.

Sproson, D. A. J., I. A. Renfrew, and K. J. Heywood (2010), A parameterization of Greenland's tip jets suitable for ocean or coupled climate models, J. Geophys. Res., 115(C8), C08022, doi: 10.1029/2009jc006002.

Stepanenko, V. M., P. M. A. Miranda, and V. N. Lykosov (2006), Numerical modeling of mesoscale interaction of the atmosphere with the hydrologically inhomogeneous land (in Russian), Computational technologies, 11(3), 129-136. 
Sullivan, P. P., and E. G. Patton (2011), The Effect of Mesh Resolution on Convective Boundary Layer Statistics and Structures Generated by Large-Eddy Simulation, J. Atmos. Sci., 68(10), 2395-2415, doi: 10.1175/jas-d-10-05010.1.

Tennekes, H. (1973), A Model for the Dynamics of the Inversion Above a Convective Boundary Layer, J. Atmos. Sci., 30(4), 558-567, doi: 10.1175/15200469(1973)030<0558:amftdo>2.0.co;2.

Tjernstrom, M., et al. (2005), Modelling the arctic boundary layer: An evaluation of six ARCMIP regional-scale models using data from the SHEBA project, Boundary-Layer Meteorol., 117(2), 337-381, doi: DOI 10.1007/s10546-004-7954-z.

Troen, I. B., and L. Mahrt (1986), A simple model of the atmospheric boundary layer; sensitivity to surface evaporation, Boundary-Layer Meteorol., 37(1), 129-148, doi: $10.1007 /$ bf00122760.

Venkatesh, S., and M. B. Danard (1978), A model for computing small-scale wind variations over a water surface, Boundary-Layer Meteorol., 14(1), 35-57, doi: 10.1007/bf00123988.

Vihma, T., J. Hartmann, and C. Lüpkes (2003), A Case Study Of An On-Ice Air Flow Over The Arctic Marginal Sea-Ice Zone, Boundary-Layer Meteorol., 107(1), 189-217, doi: $10.1023 / \mathrm{a}: 1021599601948$.

Vihma, T., C. Lüpkes, J. Hartmann, and H. Savijärvi (2005), Observations and Modelling of Cold-air Advection over Arctic Sea Ice, Boundary-Layer Meteorol., 117(2), 275-300 doi: $10.1007 /$ s10546-004-6005-0.

Wacker, U., K. Potty, C. Lüpkes, J. Hartmann, and M. Raschendorfer (2005), A Case Study on a Polar Cold Air Outbreak over Fram Strait using a Mesoscale Weather 
Prediction Model,Boundary-Layer Meteorol., 117(2), 301-336, doi: 10.1007/s10546-0052189-1.

Wai, M. M.-K., and S. A. Stage (1989), Dynamical analyses of marine atmospheric boundary layer structure near the Gulf Stream oceanic front, Quart. J. of the Roy. Meteor. Soc, 115(485), 29-44, doi: 10.1002/qj.49711548503.

Walters, M. K. (2000), commentary and analysis: Comments on "The Differentiation between Grid Spacing and Resolution and Their Application to Numerical Modeling", Bull. Amer. Meteor. Soc., 81(10), 2475-2477, doi: 10.1175/15200477(2000)081<2475:caacot>2.3.co;2.

Wu, J. (1980), Wind-Stress coefficients over Sea surface near Neutral Conditions: A Revisit, J. Phys. Oceanogr., 10(5), 727-740, doi: 10.1175/15200485(1980)010<0727:wscoss $>2.0 . \operatorname{co} ; 2$.

Wyngaard, J. C. (2004), Toward Numerical Modeling in the "Terra Incognita", J. Atmos. Sci., 61(14), 1816-1826, doi: 10.1175/1520-0469(2004)061<1816:TNMITT>2.0.CO;2.

Xue, M. (2000), High-order monotonic numerical diffusion and smoothing, Mon. Wea. Rev., 128, $2853-2864$.

Young, G. S., D. A. R. Kristovich, M. R. Hjelmfelt, and R. C. Foster (2002), Rolls, Streets, Waves, and More: A Review of Quasi-Two-Dimensional Structures in the Atmospheric Boundary Layer, Bull. Amer. Meteor. Soc., 83(7), 997-1001, doi: 10.1175/15200477(2002)083<0997:rswama>2.3.co;2.

Zalesak, S. T. (1979), Fully multidimensional flux-corrected transport algorithms for fluids, J. Comput. Phys., 31(3), 335-362, doi: 10.1016/0021-9991(79)90051-2.

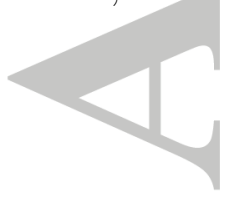



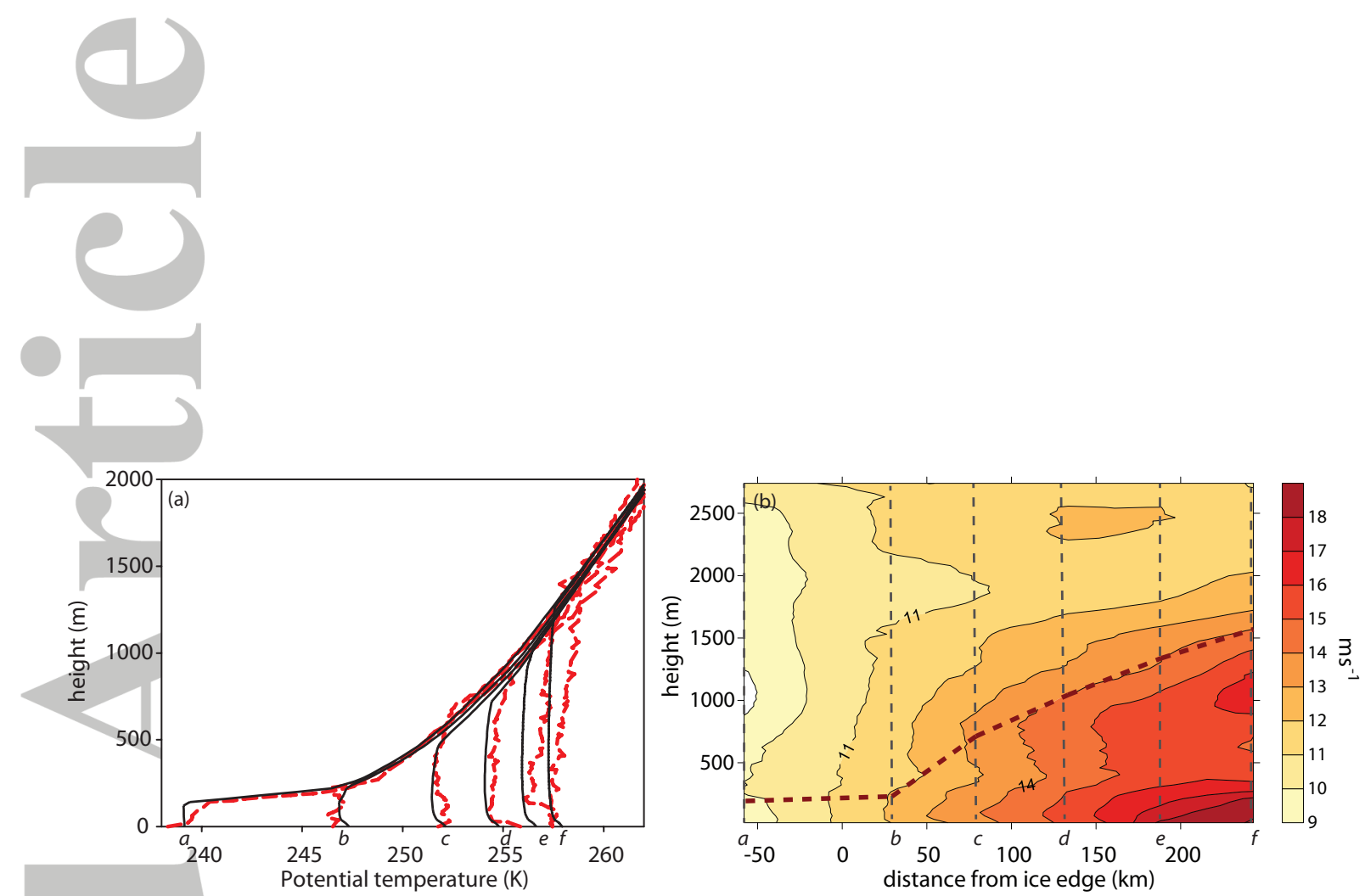

Figure 1. Vertical profiles of potential temperature (panel $a$ ) at different distances from the ice edge (the red dashed line represents observations; the solid black line are NH3D results); Vertical cross-section in north-south direction of absolute wind speed (panel $b$; dark red dashed line represents the ABL height diagnosed from observed potential temperature profiles). The location of the dropsonde releases is labeled as: $a--58 \mathrm{~km}, b-33 \mathrm{~km}, c-80 \mathrm{~km}, d-132 \mathrm{~km}, e-188$ $\mathrm{km}, f-243 \mathrm{~km}$. 


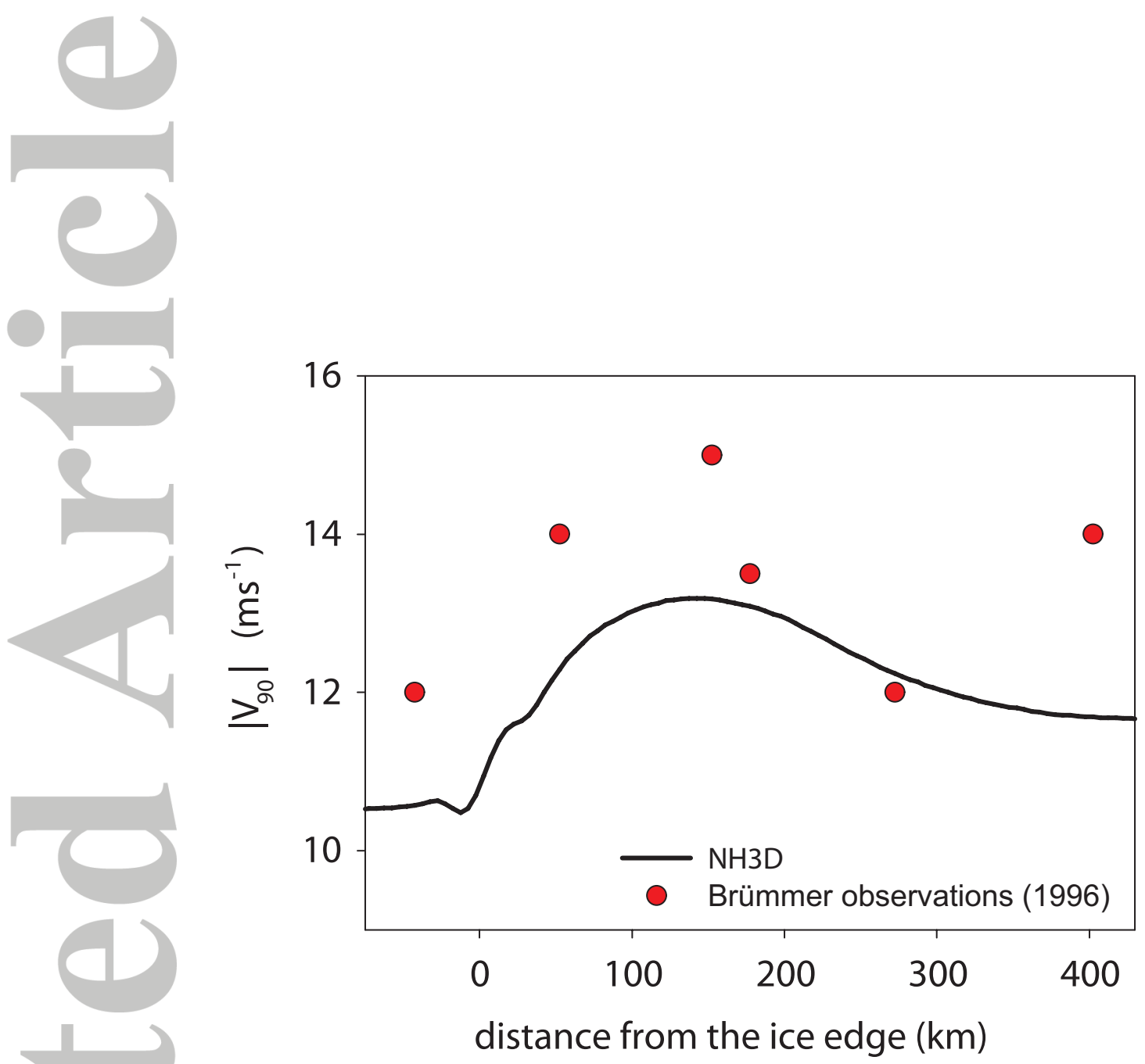

Figure 2. Absolute value of wind speed at $90 \mathrm{~m}$ height as function of distance from the ice edge. Rounds correspond to the spatially averaged aircraft observations during flight legs parallel to the ice edge from Brümmer [1996]; the averaging length is about $50 \mathrm{~km}$. Solid line represents the results of NH3D.

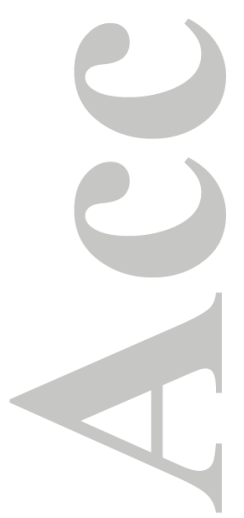


$\Theta(\mathrm{K})$

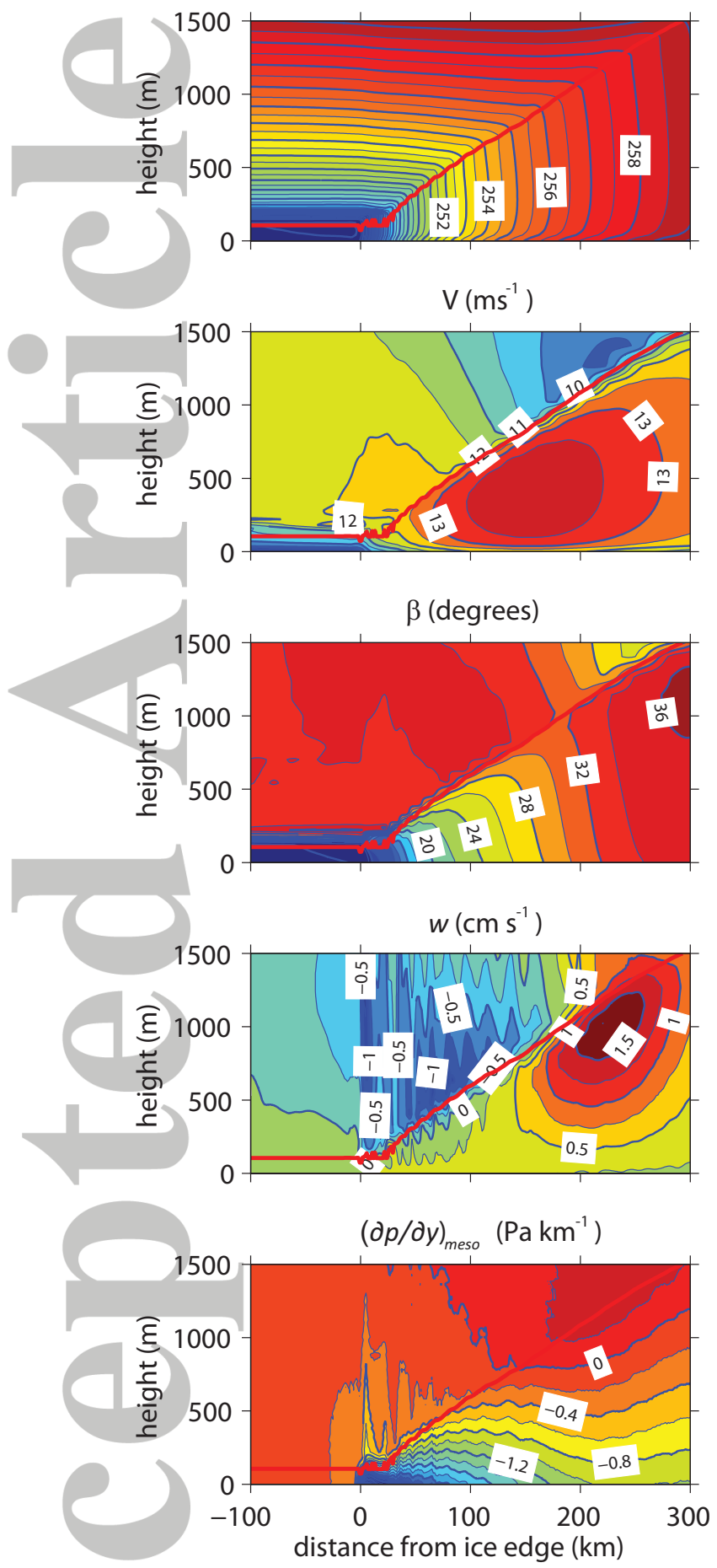

$\Theta(\mathrm{K})$
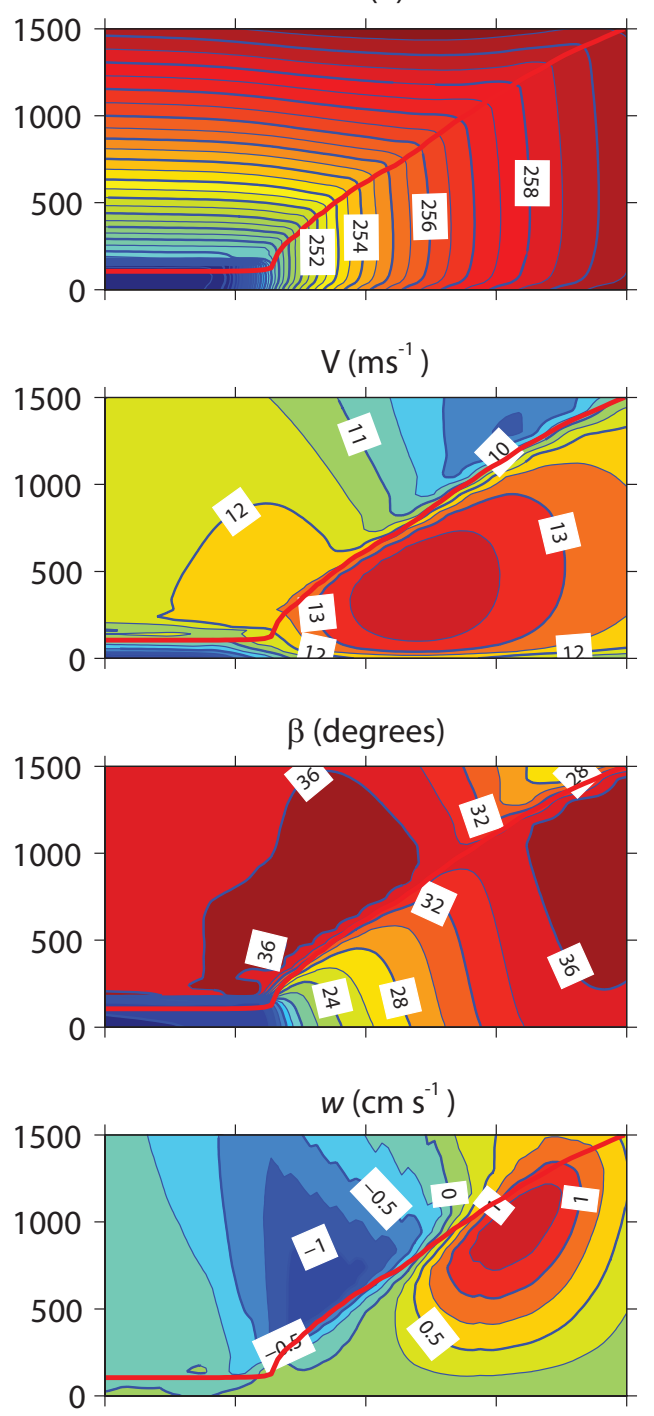

$(\partial p / \partial y)_{\text {meso }}\left(\mathrm{Pa} \mathrm{km}^{-1}\right)$

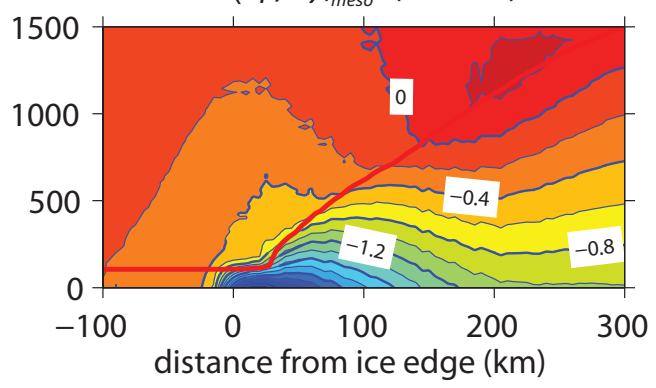

Figure 3. Vertical cross-sections of potential temperature $\theta(\mathrm{K})$, absolute horizontal wind speed $\mathrm{V}\left(\mathrm{ms}^{-1}\right)$, horizontal wind direction $\beta$ (degrees), vertical wind speed $w\left(\mathrm{~cm} \mathrm{~s}^{-1}\right)$ and mesoscale pressure gradient $(\partial p / \partial y)_{\text {meso }}\left(\mathrm{Pa} \mathrm{km}^{-1}\right)$. Results of the runs with $\Delta y=1.25 \mathrm{~km}\left(L_{M I Z} \approx 12 \mathrm{~km}\right)$ and $\Delta y=5 \mathrm{~km}\left(L_{M I Z}=0 \mathrm{~km}\right)$ are on the left and right panels, respectively. The red line represents the diagnosed ABL height. Air flow is directed from left to right.

(C)2013 American Geophysical Union. All Rights Reserved. 

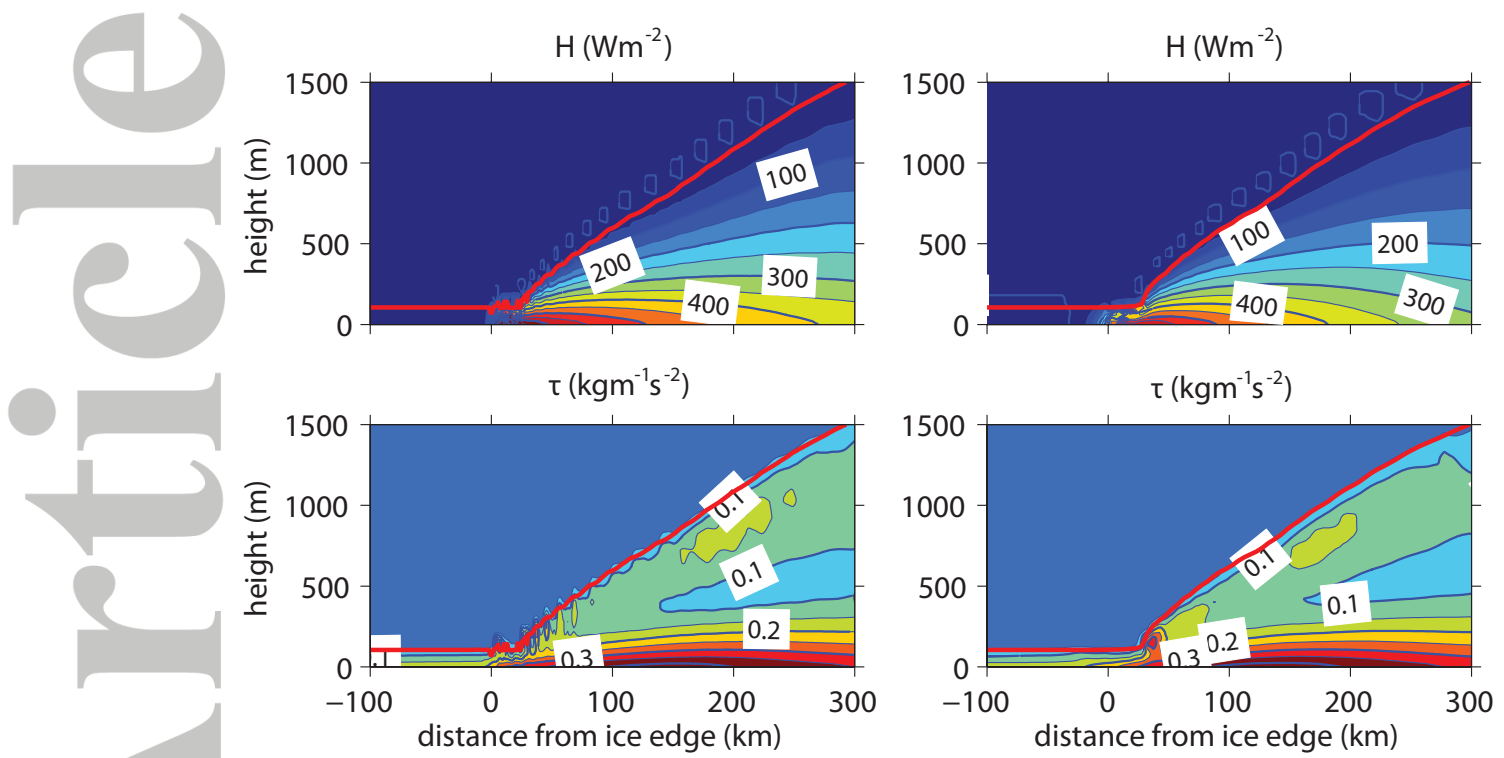

Figure 4. As in Figure 3, but for vertical cross-sections of sensible heat flux $\mathrm{H}\left(\mathrm{Wm}^{-2}\right)$ and momentum flux $\tau\left(\mathrm{kgm}^{-1} s^{-2}\right)$.
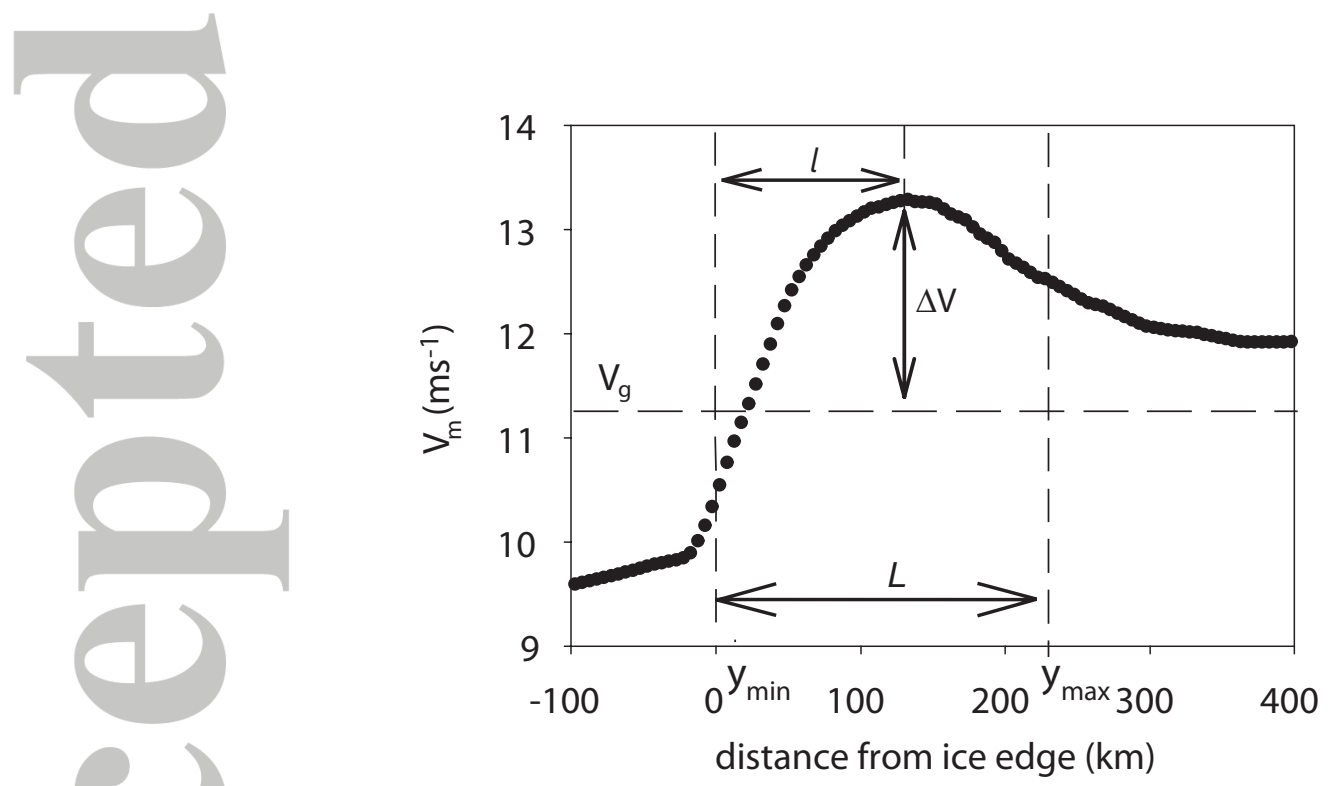

Figure 5. Absolute values of wind speed $\mathbf{V}_{\mathbf{m}}$ averaged over the ABL depth as function of the distance from the ice edge. $L$ is the characterisitic horizontal scale of the IBJ and $\Delta V$ is a characteristic velocity scale of the IBJ. $l$ is the distance of the wind speed maximum from the ice edge. 


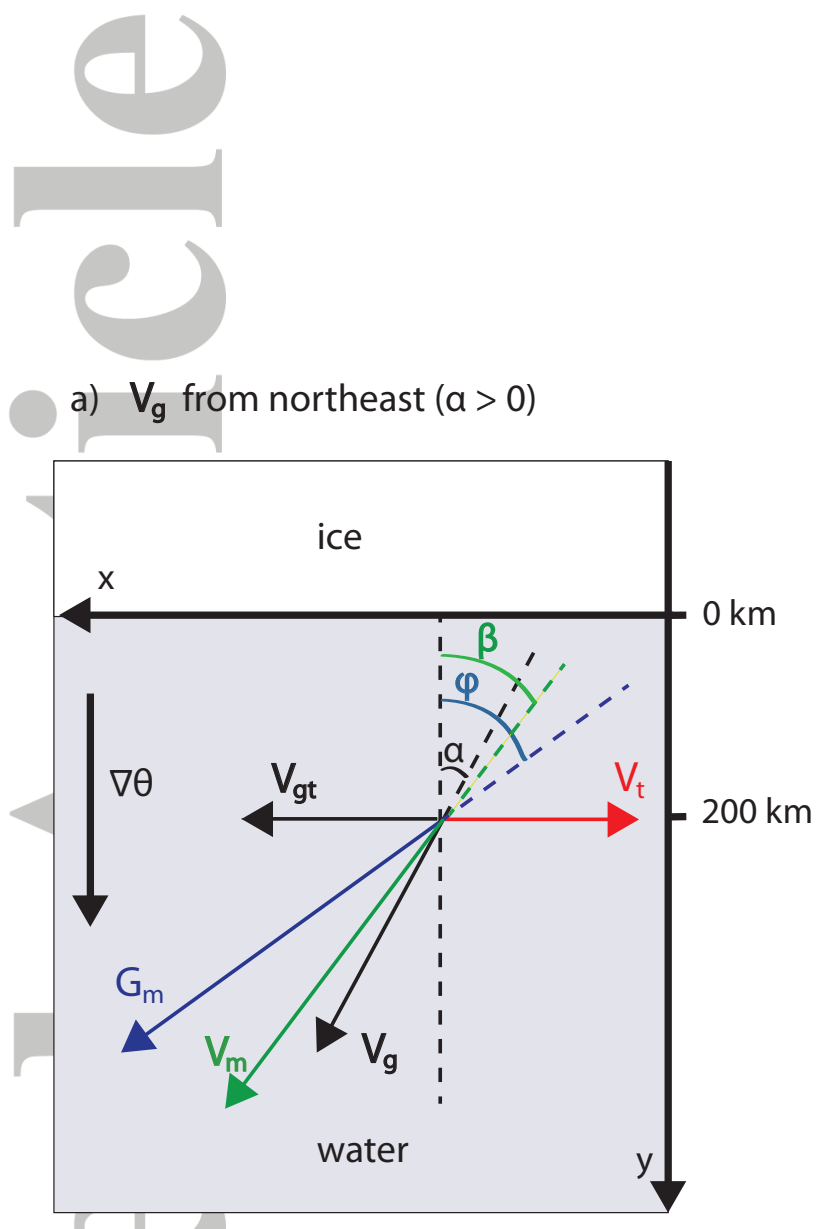

b) $V_{g}$ from northwest $(a<0)$

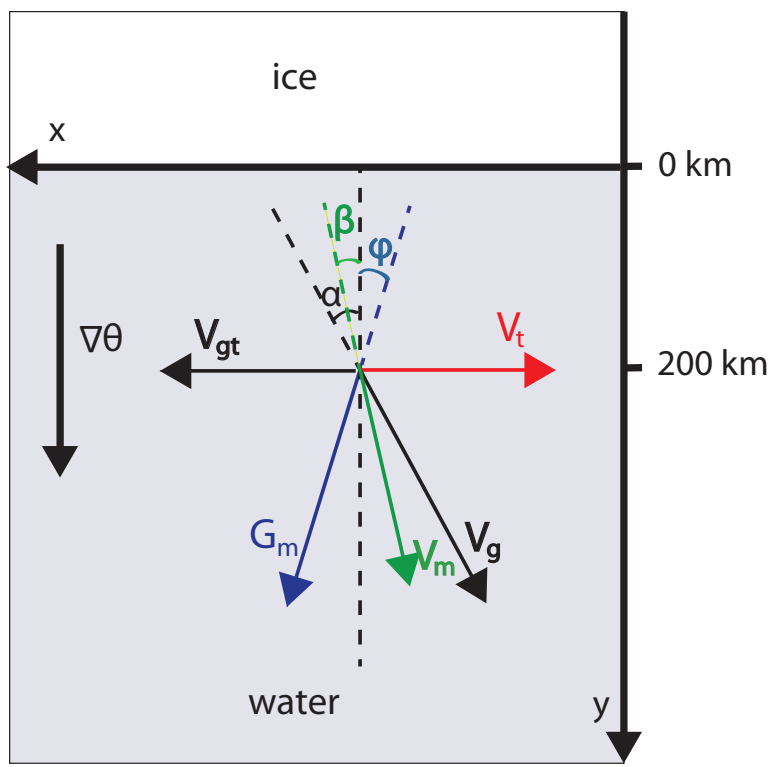

Figure 6. Large-scale geostrophic wind $\mathbf{V}_{\mathbf{g}}$, mean geostrophic ABL wind $\mathbf{G}_{\mathbf{m}}$, ABL-mean wind speed $\mathbf{V}_{\mathbf{m}}$ and corresponding angles $\alpha, \phi$ and $\beta$ relative to the direction orthogonal to the ice edge ( $\alpha, \phi, \beta>0$ when the corresponding vectors have the easterly component). $\mathbf{V}_{\mathbf{g t}}$ is the ABL-vertically averaged thermally induced geostrophic wind and $\mathbf{V}_{\mathbf{T}}$ is the ABL-averaged thermal wind, as defined in Appendix A. The scheme is shown for constant $\left|\mathbf{V}_{\mathbf{g}}\right|$ and $\left|\mathbf{V}_{\mathbf{g t}}\right|$ but symmetric directions of $\mathbf{V}_{\mathbf{g}}$ : a) from northeast $(\alpha>0)$; b) from northwest $(\alpha<0)$. 

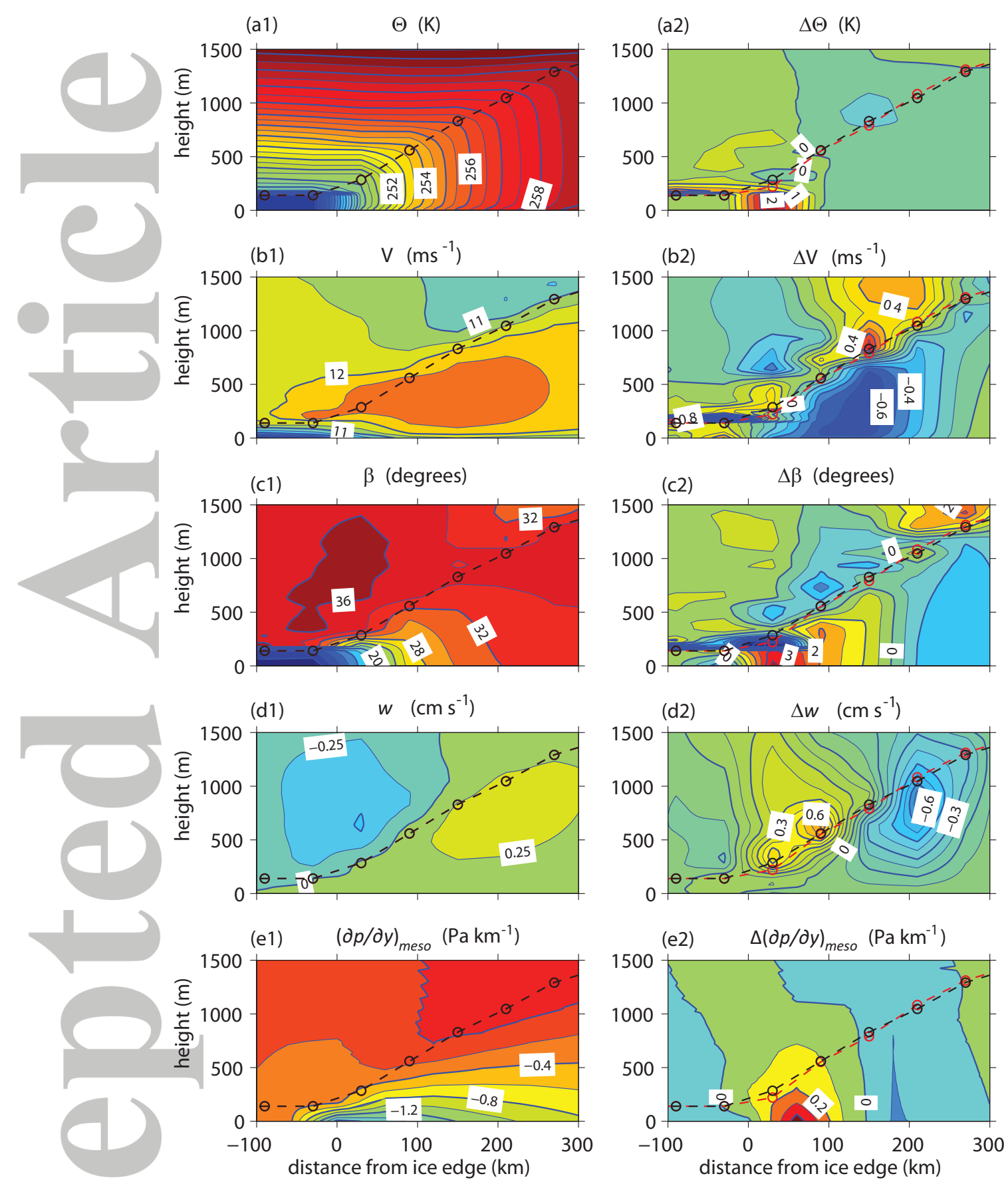

Figure 7. Vertical cross-sections of potential temperature $\Theta(\mathrm{K})$, absolute horizontal wind speed $\mathrm{V}\left(\mathrm{ms}^{-1}\right)$, wind speed direction $\beta$ (degrees), vertical wind speed $w\left(\mathrm{~cm} \mathrm{~s}^{-1}\right)$, and mesoscale pressure gradient $(\partial p / \partial y)_{\text {meso }}\left(\mathrm{Pa} \mathrm{km}^{-1}\right)$ based on NH3D results with $\Delta y=60 \mathrm{~km}$ (left column). Vertical cross-sections of the difference between the corresponding fields from coarse- and highresolution runs (right column). The dashed line represents the ABL height (red: high-resolution run, blue: coarse-resolution run). 

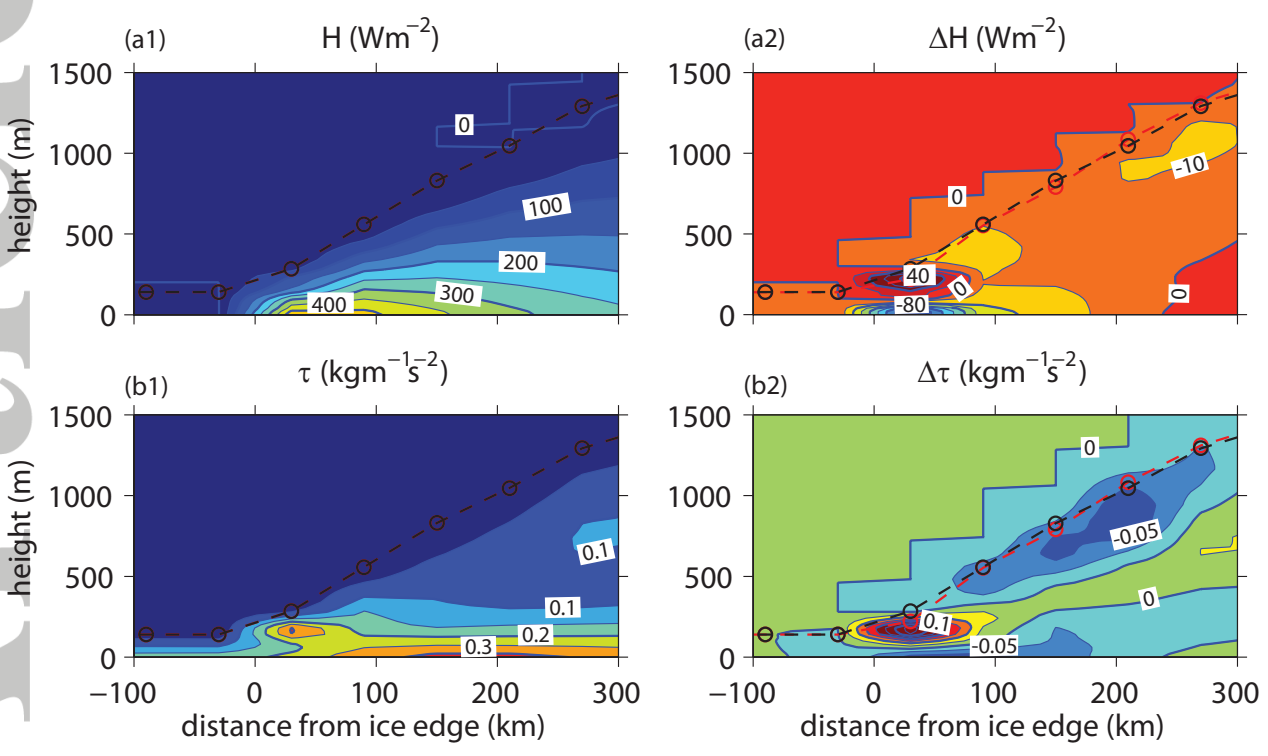

Figure 8. The same as in Figure 7, but for the fluxes of sensible heat $\mathrm{H}\left(\mathrm{Wm}^{-2}\right)$ and momentum
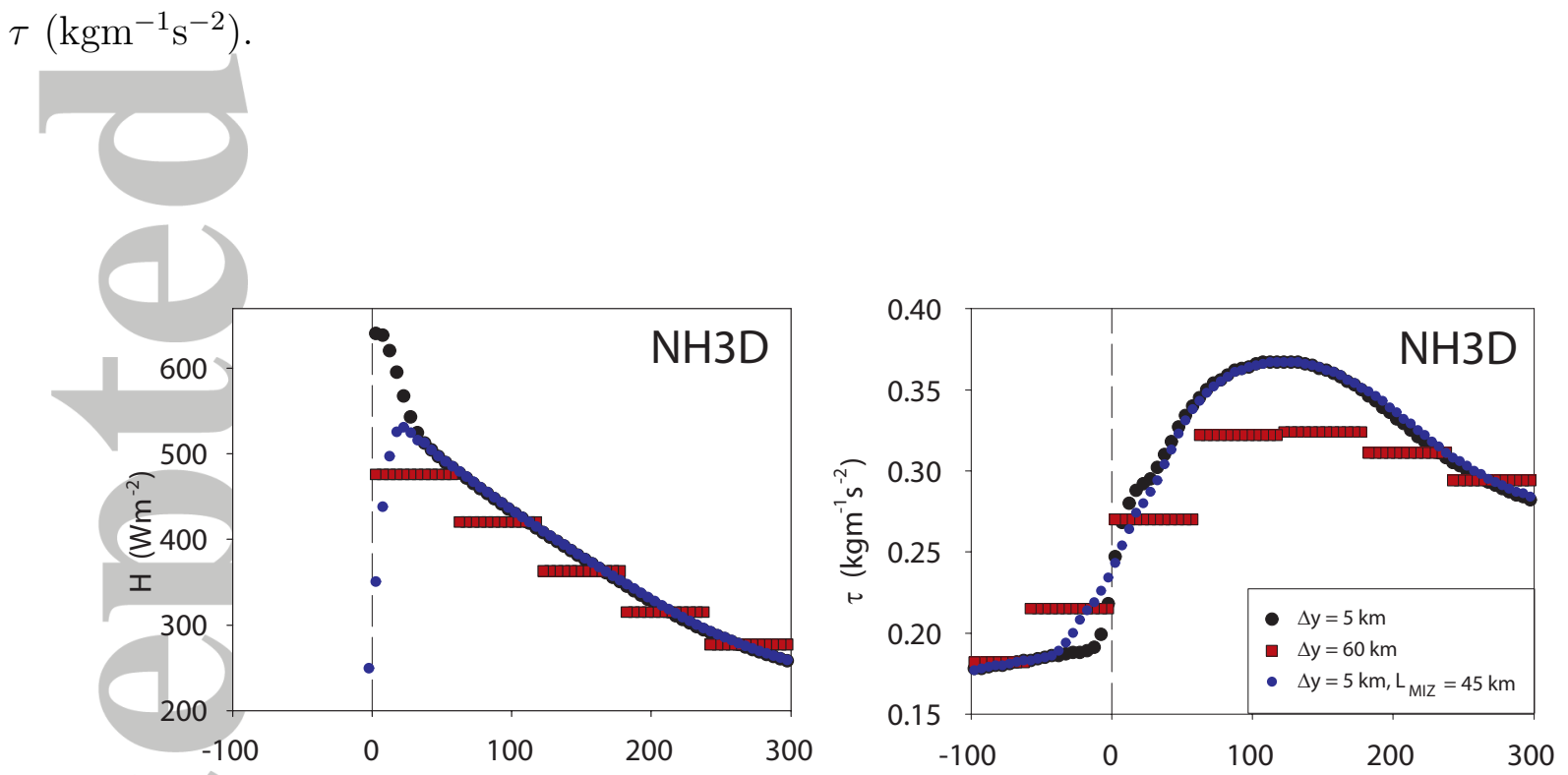

Figure 9. Surface fluxes of heat $H\left(\mathrm{Wm}^{-2}\right)$ and momentum $\tau\left(\mathrm{kgm}^{-1} \mathrm{~s}^{-2}\right)$ as function of the distance from the ice edge obtained from NH3D with $\Delta y=5 \mathrm{~km}$ (black dots) and $\Delta y=60 \mathrm{~km}$ (red squares). Results from the NH3D run with $\Delta y=5 \mathrm{~km}$ and $L_{M I Z}=45 \mathrm{~km}$ (blue dots) are shown as functions of the distance from the MIZ center. 

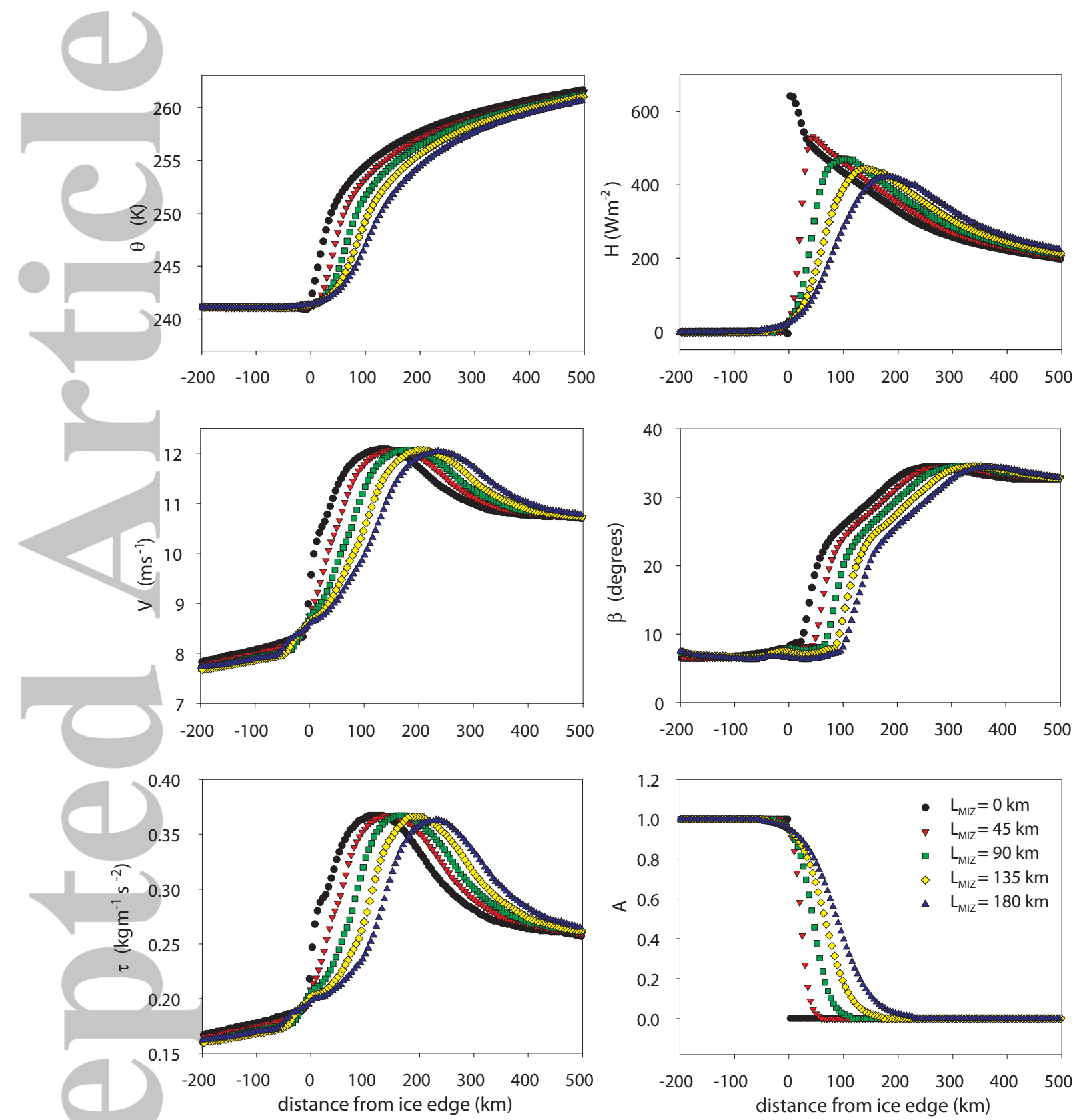

Figure 10. Results from NH3D for potential temperature $\theta(\mathrm{K})$, sensible heat flux $H\left(\mathrm{Wm}^{-2}\right)$, absolute wind speed $V\left(\mathrm{~ms}^{-1}\right)$, wind direction $\beta$ (degrees), momentum flux $\tau\left(\mathrm{kgm}^{-1} \mathrm{~s}^{-2}\right)$ at 10 meter height, and sea-ice concentration $A$ as functions of the distance from the ice edge defined as the position with $A=0.95$.

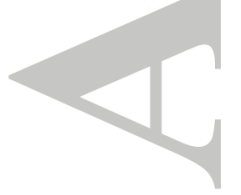

(C)2013 American Geophysical Union. All Rights Reserved. 

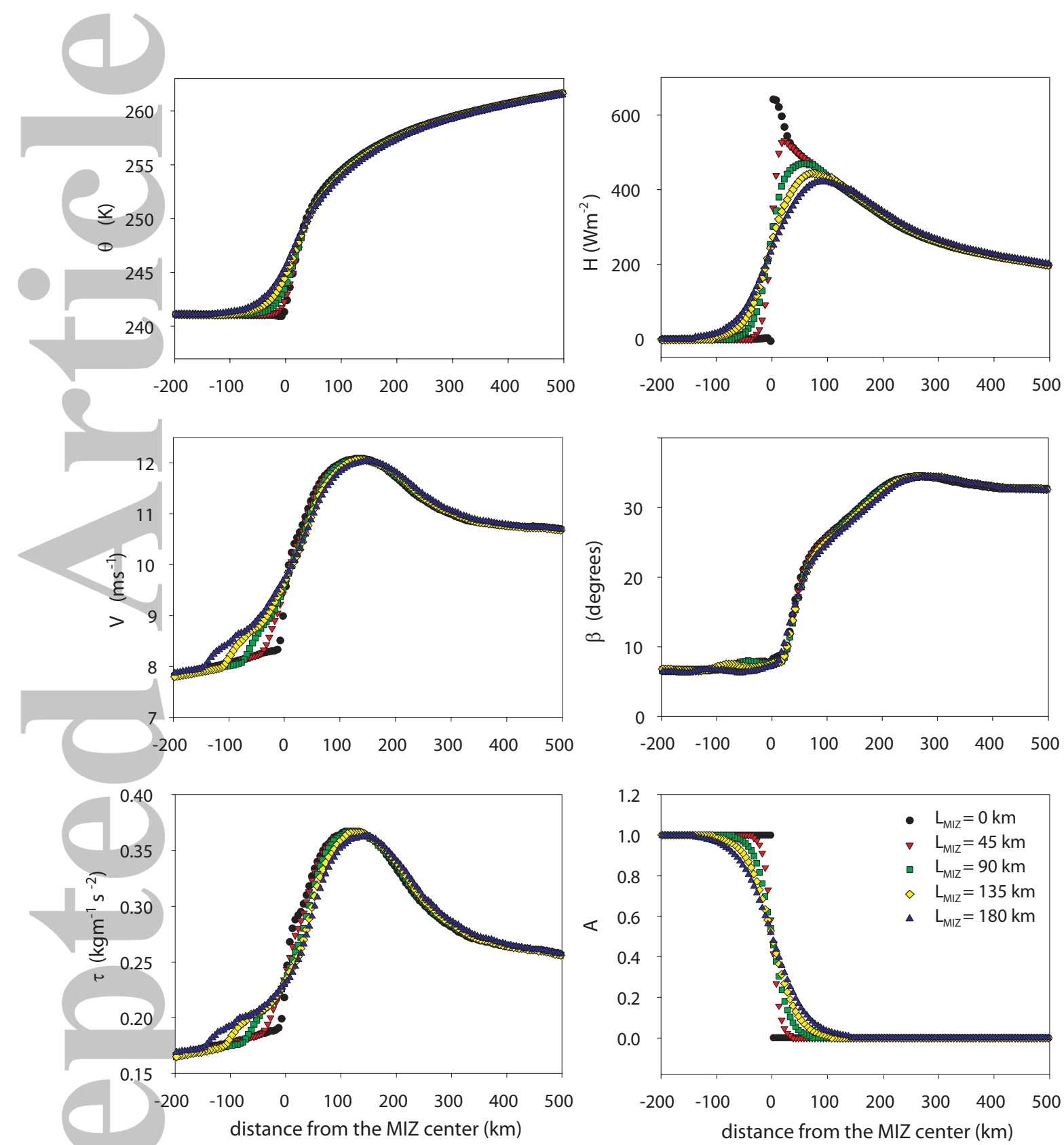

Figure 11. Results of NH3D for potential temperature $\theta(\mathrm{K})$, sensible heat flux $H\left(\mathrm{Wm}^{-2}\right)$, absolute wind speed $V\left(\mathrm{~ms}^{-1}\right)$, wind direction $\beta$ (degrees), momentum flux $\tau\left(\mathrm{kgm}^{-1} \mathrm{~s}^{-2}\right)$ at 10 meter height, and sea-ice concentration $A$ as functions of the distance from the MIZ center where $A=0.5$. 

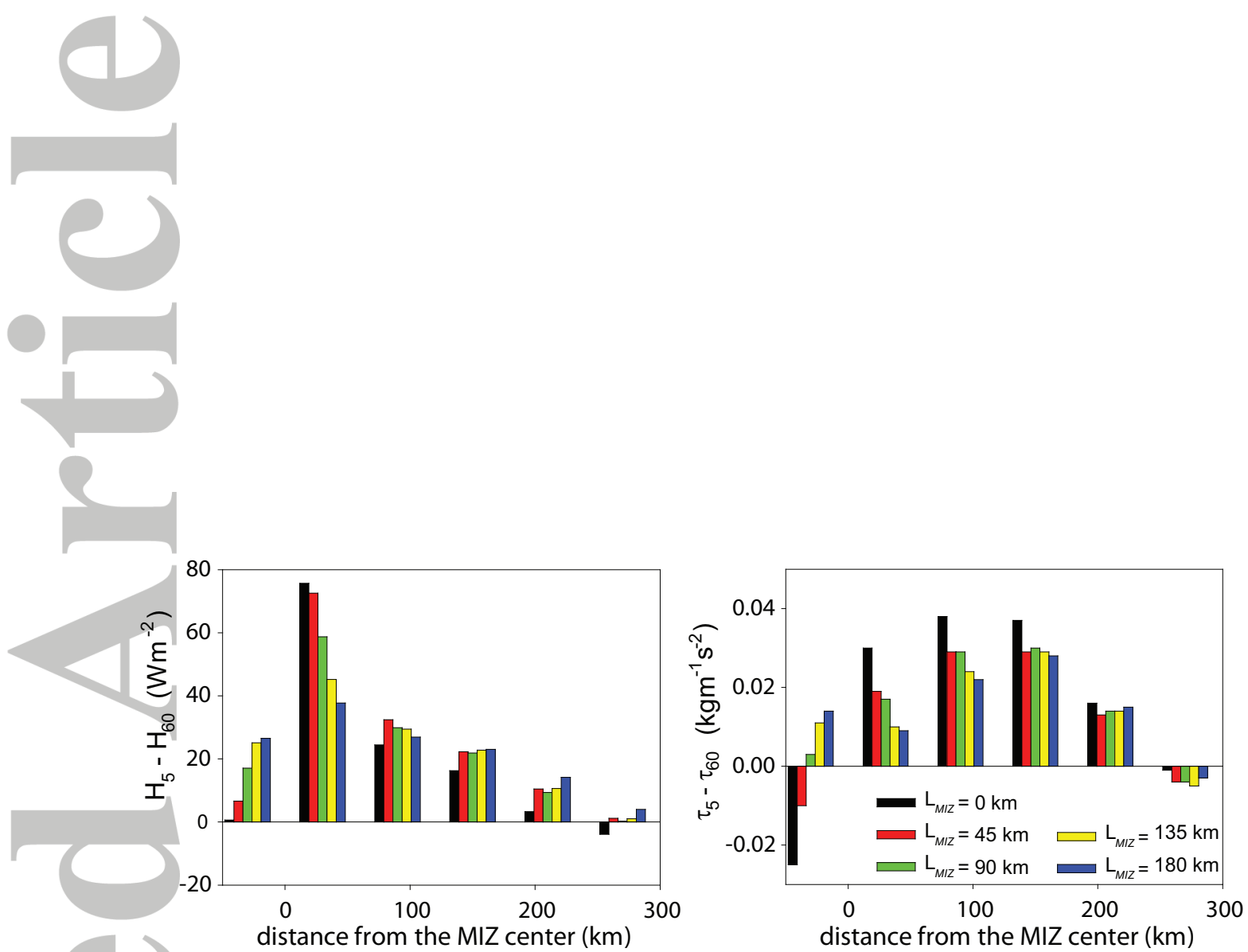

Figure 12. Difference of surface fluxes of heat $H$ and momentum $\tau$ between NH3D results with coarse and high resolution as functions of the distance from the MIZ center for different values of $L_{M I Z}$.

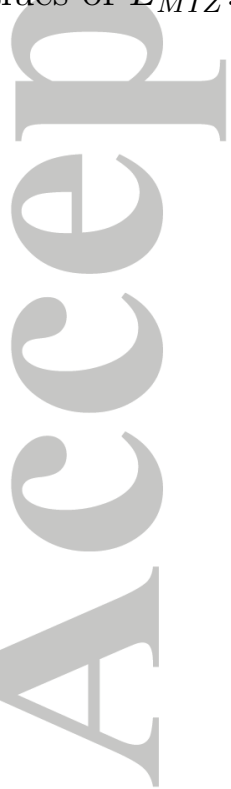



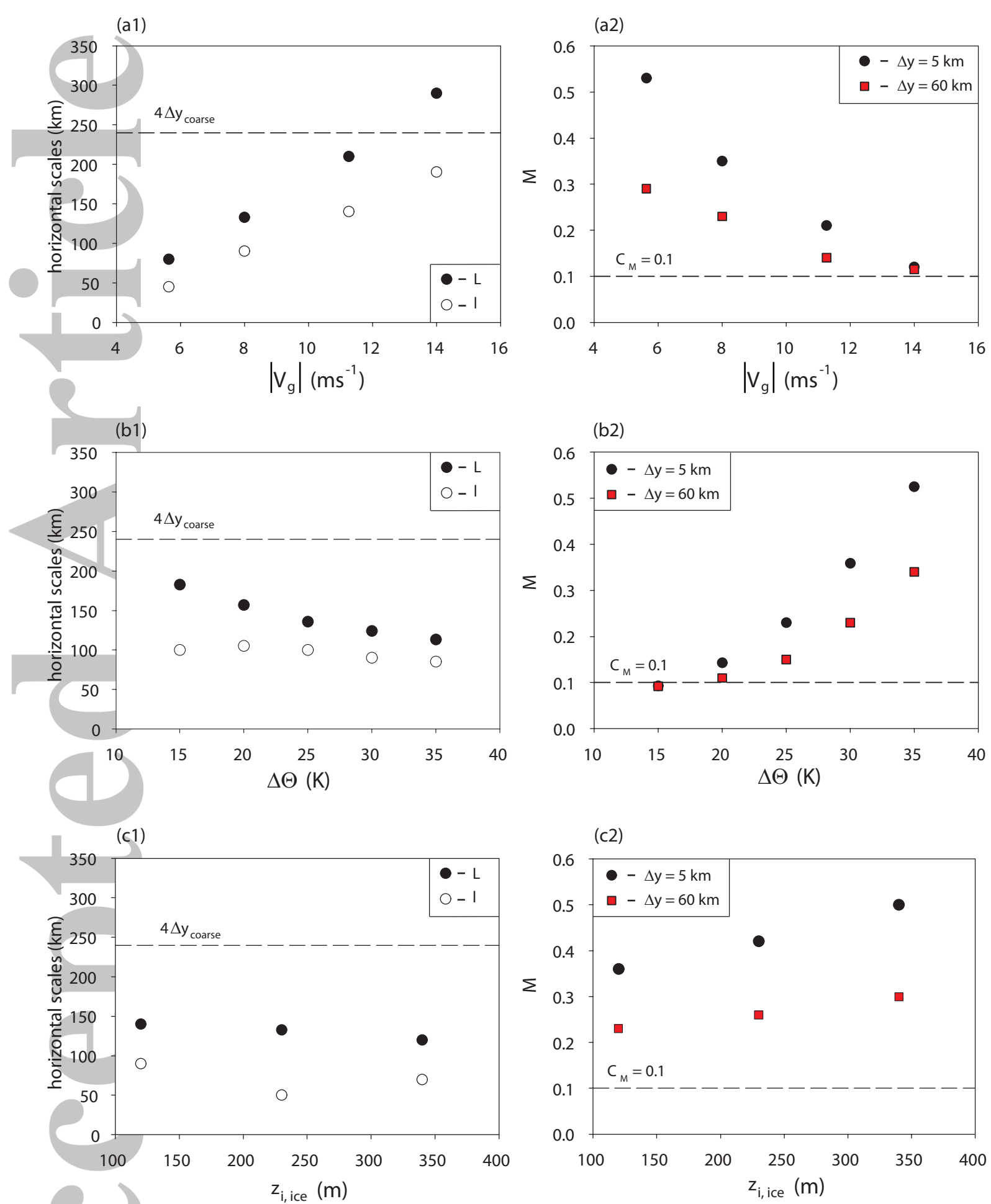

Figure 13. Characteristic scales $L$ and $l$ of the IBJ and the IBJ strength $M$ as functions of the absolute geostrophic wind speed $\left|\mathbf{V}_{\mathrm{g}}\right|$ (panels $a 1$ and $a$ 2); temperature difference between open water and sea ice $\Delta \Theta$ (panels $b 1$ and b2); height of the ABL over sea ice $z_{i, i c e}$ (panels $c 1$ and $c 2$ ). 
(a1)

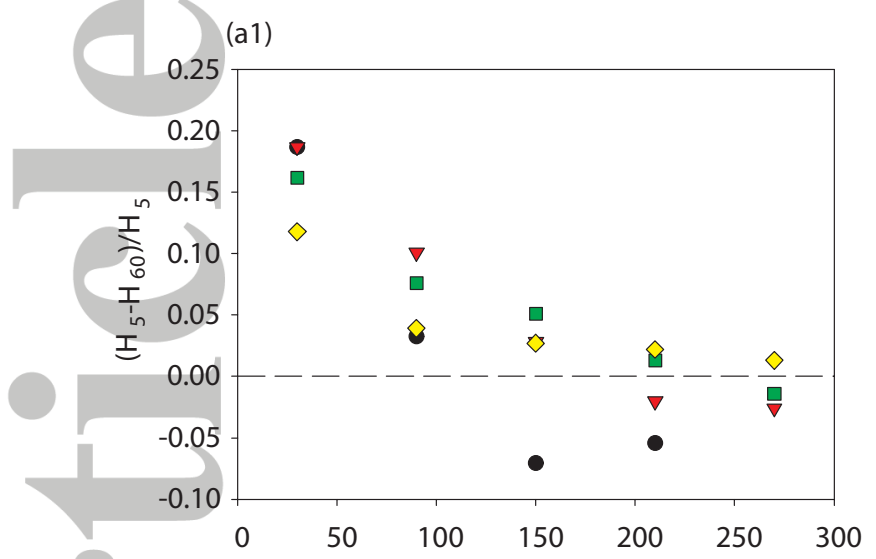

(b1)

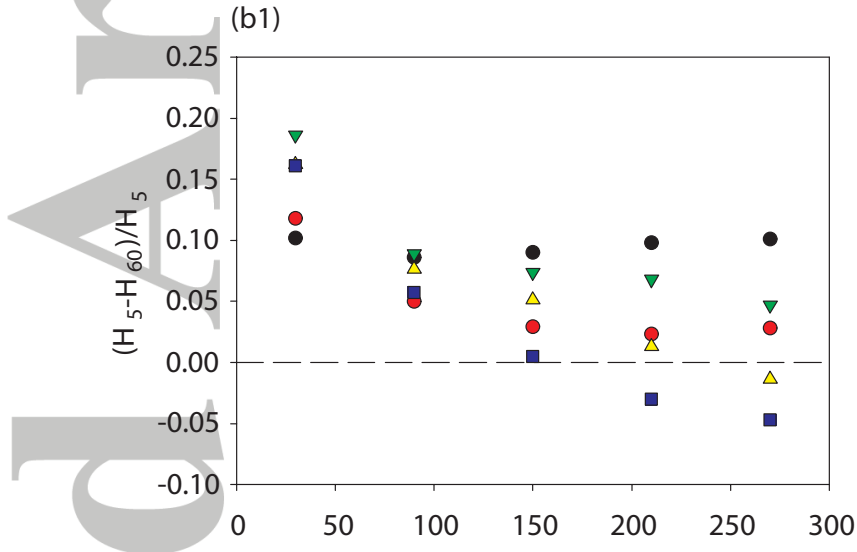

(c1)

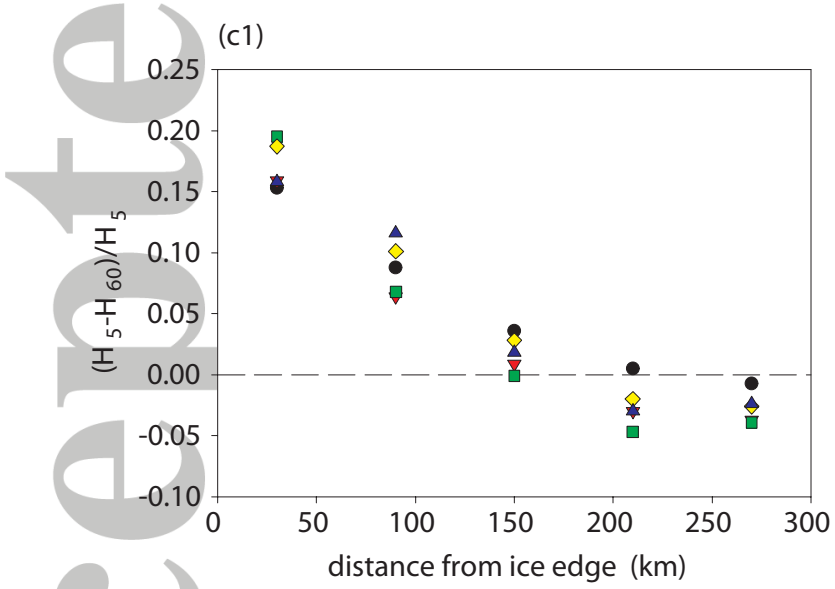

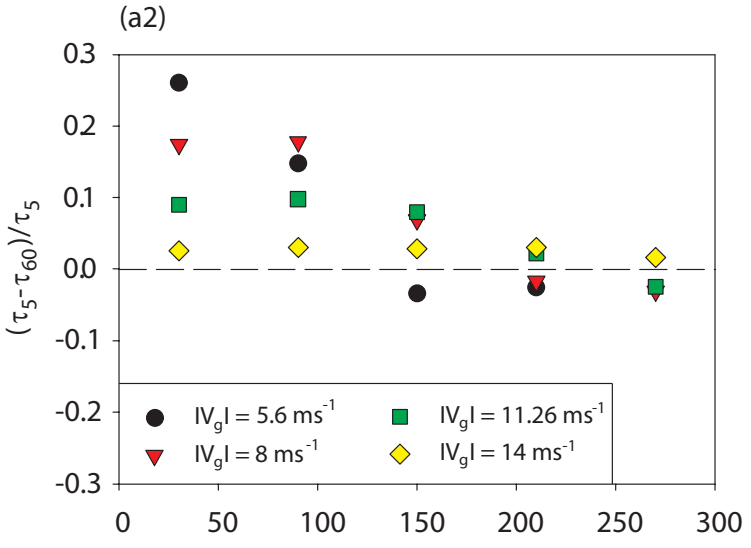

(b2)
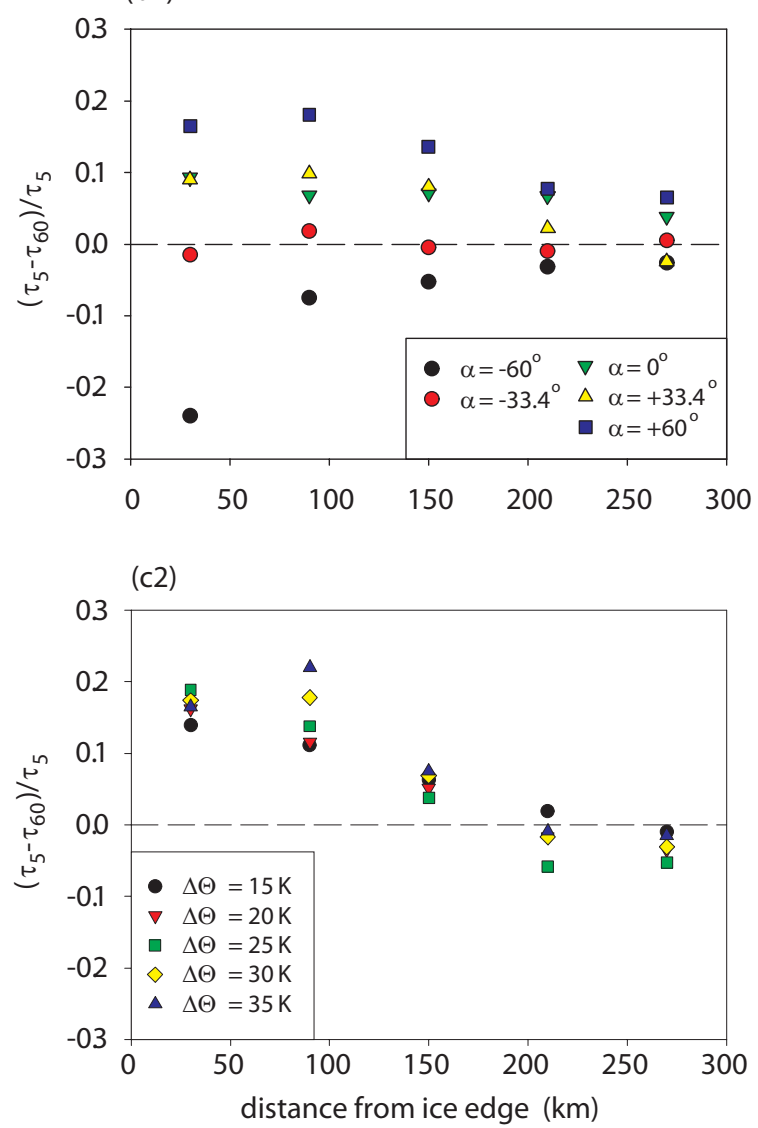

Figure 14. Normalized difference between surface fluxes of heat and momentum simulated by

NH3D with coarse and high resolution for different absolute geostrophic wind speeds $\left|\mathbf{V}_{\mathbf{g}}\right|$ (panels $a 1$ and a2), for diferent directions of geostrophic wind $\alpha$ (panels b1 and b2), and for different temperature differences between open water and sea ice $\Delta \Theta$ (panels $c 1$ and $c 2)$. 

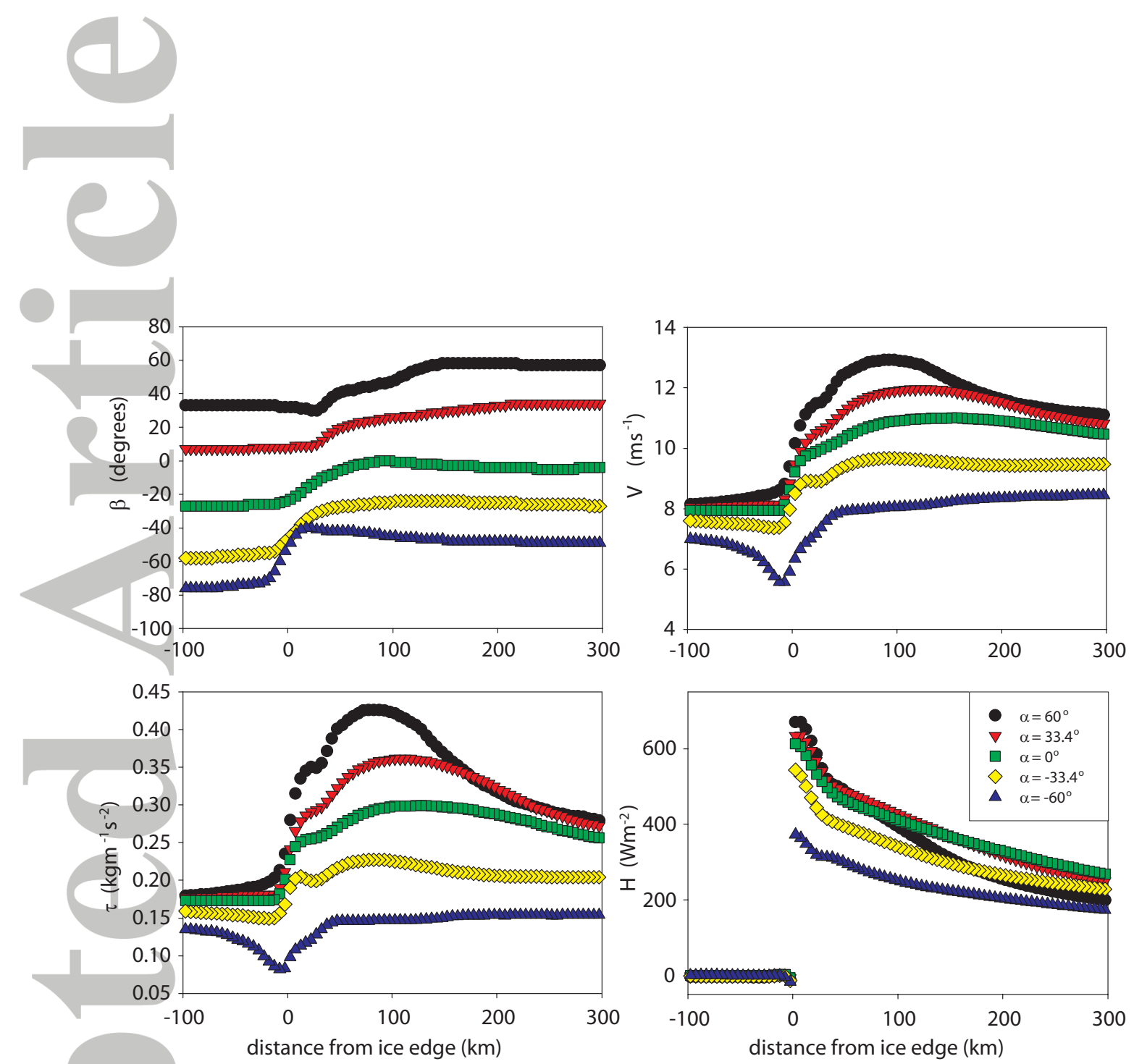

Figure 15. Direction $\beta$ and absolute values of wind $V$, surface fluxes of momentum $\tau$, and heat $H$ at $10 \mathrm{~m}$ height as function of the distance from the ice edge simulated by NH3D for different values of the large scale geostrophic wind direction $\alpha$.

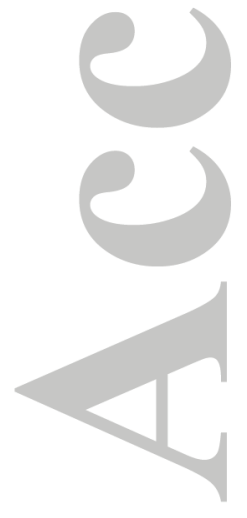

(C)2013 American Geophysical Union. All Rights Reserved. 


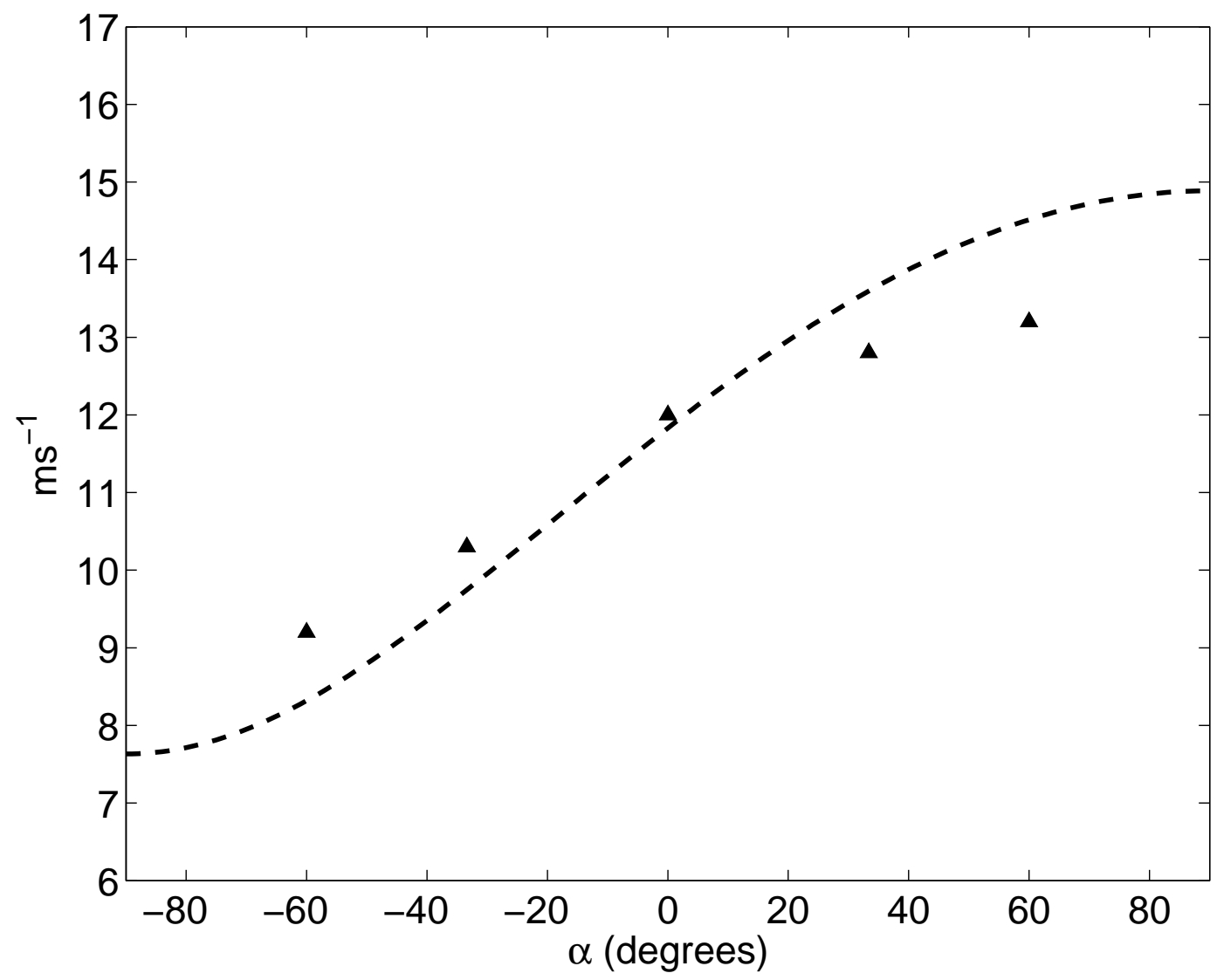

Figure 16. Absolute value of the ABL-averaged geostrophic wind speed $\left|\mathbf{G}_{\mathbf{m}}\right|$ estimated using equation (3) (dashed line) and absolute values of horizontal wind speed averaged over the ABL depth at $200 \mathrm{~km}$ downstream the ice edge from NH3D experiments for different $\alpha$.
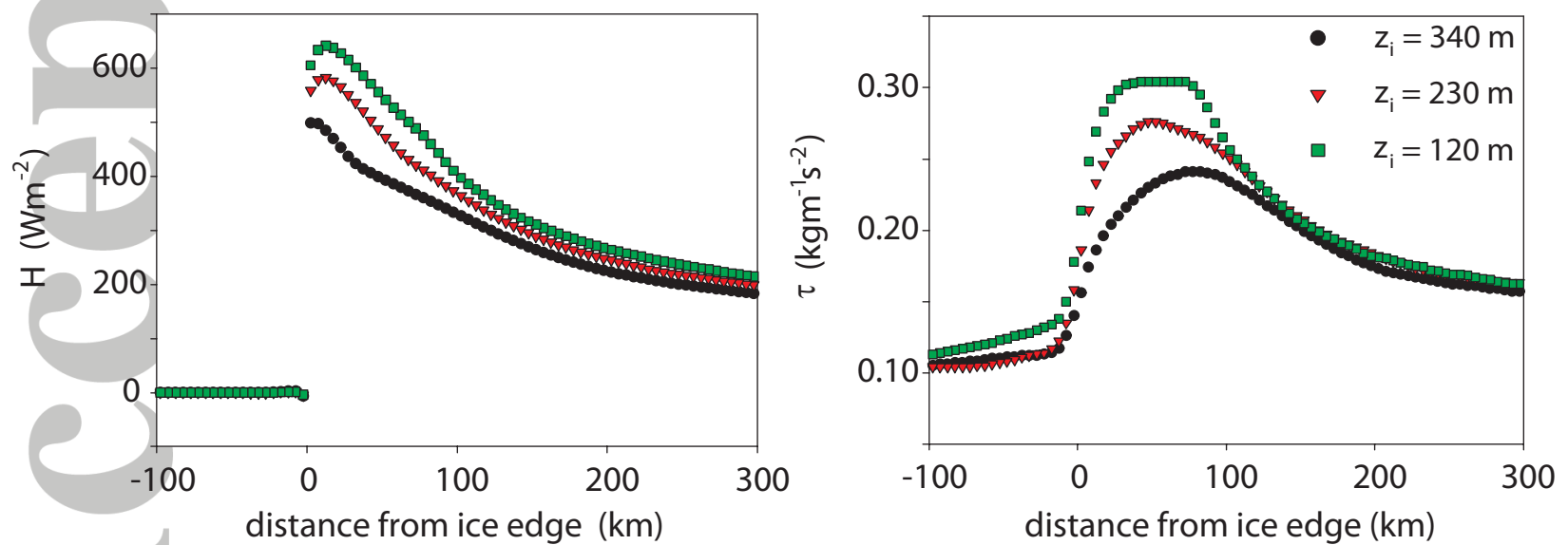

Figure 17. Surface fluxes of heat $H$ and momentum $\tau$ as functions of distance from the ice edge for different values of the ABL thickness $z_{i, i c e}$ over ice. 

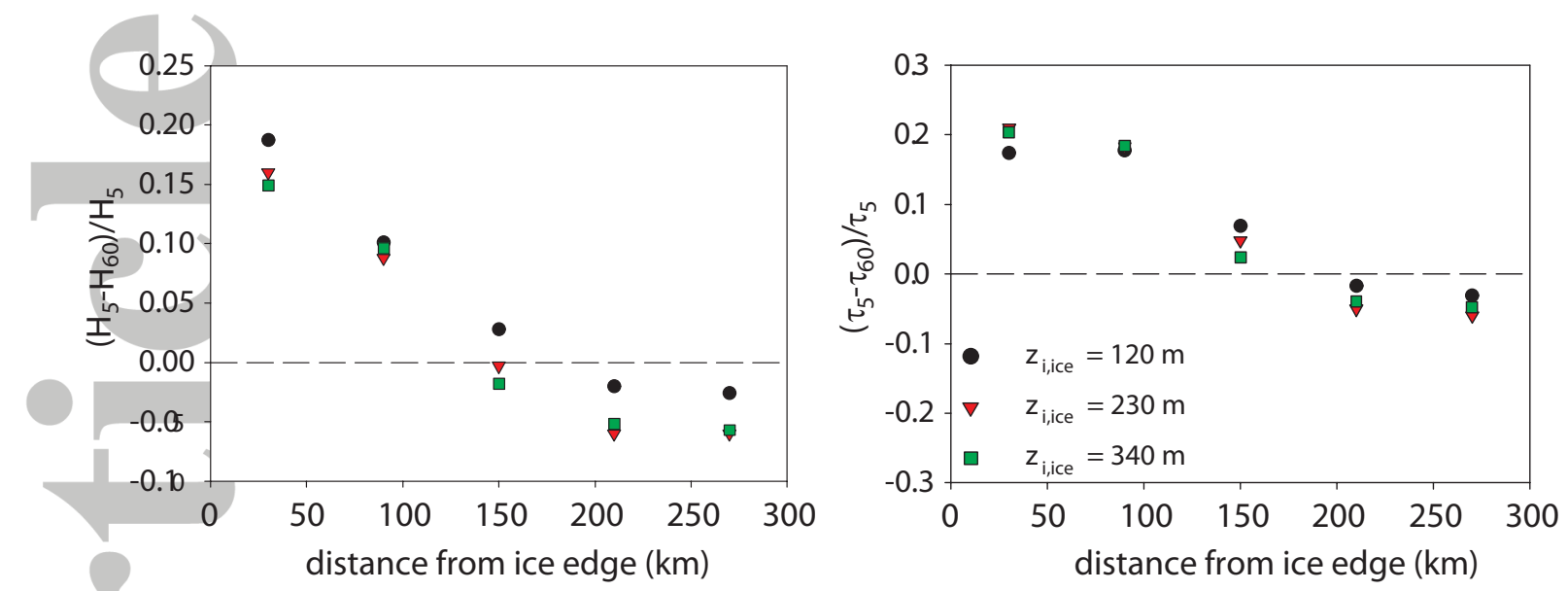

Figure 18. Normalized difference of surface fluxes of heat $H\left(\mathrm{Wm}^{-2}\right)$ and momentum $\tau$ $\left(\mathrm{kgm}^{-1} \mathrm{~s}^{-2}\right)$ between NH3D results with coarse and high resolution, as functions of distance from the ice edge for different values of $z_{i, i c e}$.
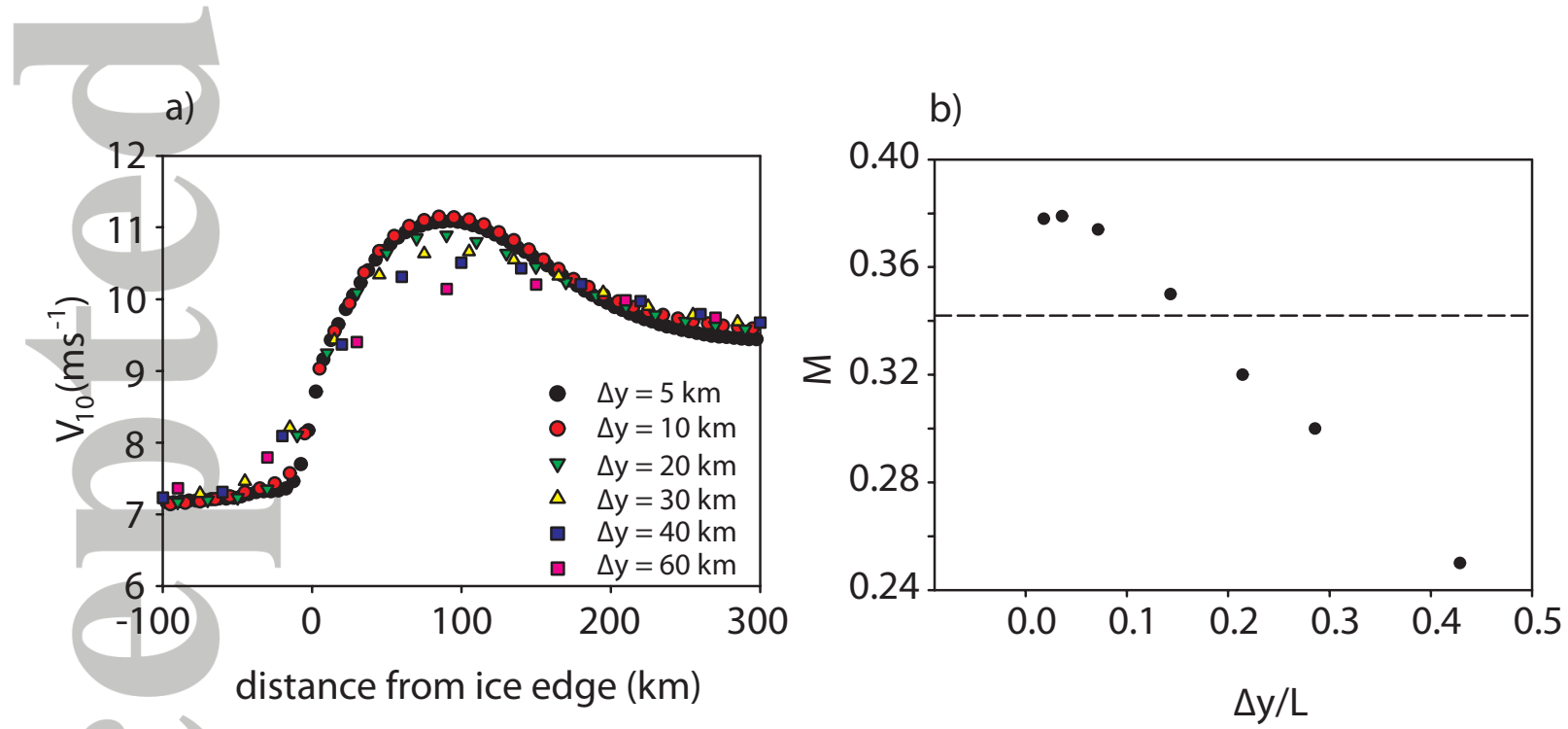

Figure 19. Absolute wind speed at $10 \mathrm{~m}$ height as function of distance from the ice edge (panel a) and the IBJ magnitude $M$ (panel $b)$. The dashed line on panel $b$ indicates the value $0.9 M$, where $M$ is taken from the experiment with $\Delta y=5 \mathrm{~km}$. Results are obtained from experiments with $\Delta y=2.5,5,10,20,30,40,60 \mathrm{~km}$. 

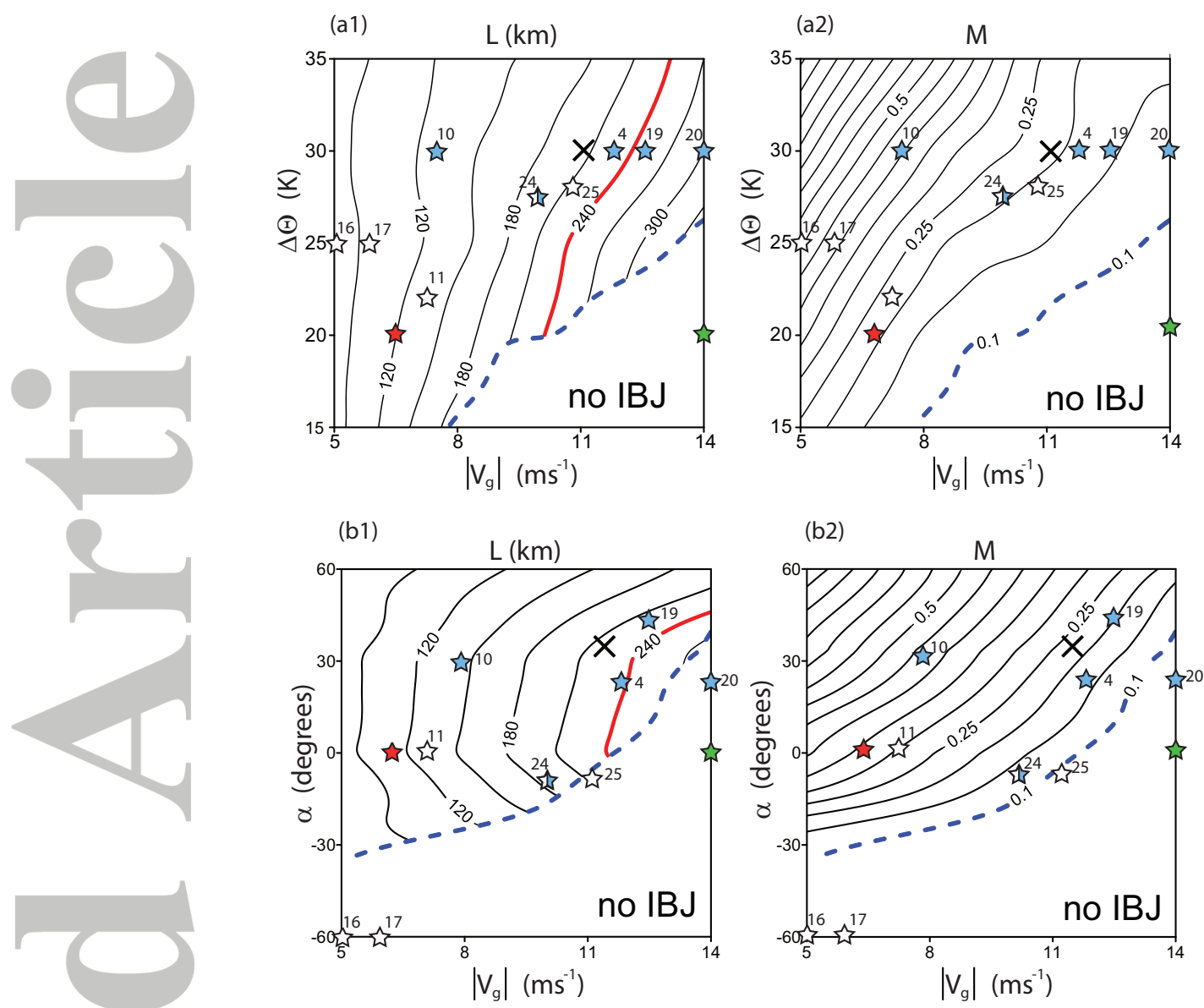

Figure 20. Horizontal scale $L$ and the IBJ strength $M$ as functions of absolute geostrophic wind speed $\left|\mathbf{V}_{\mathbf{g}}\right|$ and of the temperature difference between open water and sea ice $\Delta \Theta$ (panels $a 1$ and a2) and of $\left|\mathbf{V}_{\mathbf{g}}\right|$ and large-scale geostrophic wind direction $\alpha$ (panels $b 1$ and $\left.b 2\right)$. The red line corresponds to $L$ equal to $240 \mathrm{~km}$, indicating the critical value below which the resolution criteria (5) is not satisfied for the IBJ. The blue dashed line corresponds to $M$ equal to 0.1 , below which the IBJ is too weak to be identified. All NH3D results are obtained with $\Delta y=5 \mathrm{~km}$. The black cross corresponds to the reference run; the green star indicates the CAO observed and modelled by Wacker et al [2005]; the red star is the CAO simulated by Bechtold et al [1992]; the blue and the empty stars represent the CAO cases observed by Brümmer [1996] with IBJ and without (numbers next to the stars indicate the day of March 1993); the half-blue stars refer to the cases by Brümmer [1999] where a low-level jet was found in the ABL only in vertical profiles. 
$\Theta(\mathrm{K})$

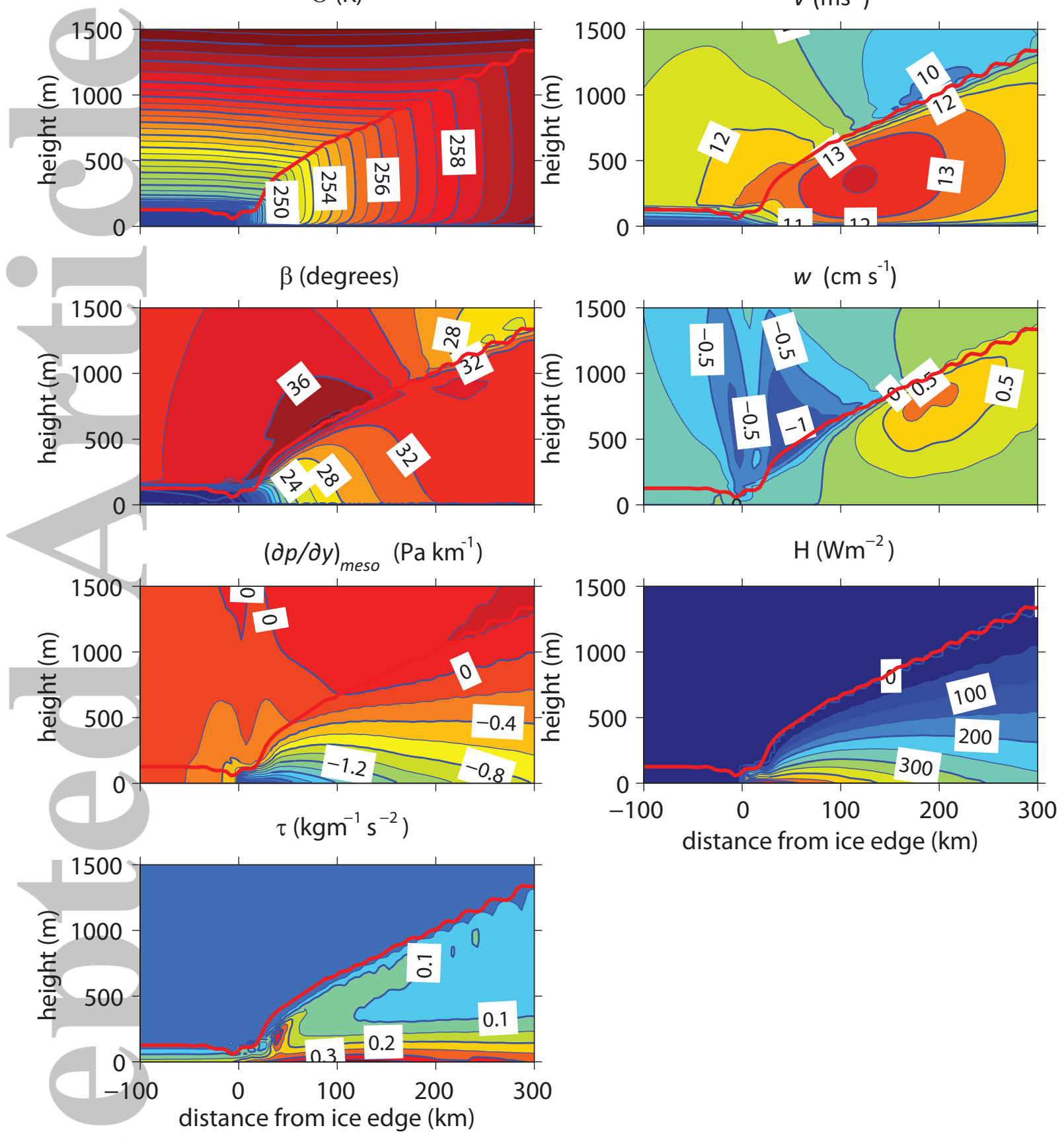

Figure 21. Vertical cross-sections of potential temperature $\Theta(\mathrm{K})$, absolute horizontal wind speed $V\left(\mathrm{~ms}^{-1}\right)$, horizontal wind direction $\beta$ (degrees), vertical wind speed $w\left(\mathrm{~cm} \mathrm{~s}^{-1}\right)$, mesoscale pressure gradient $\left.(\partial p / \partial y)_{\text {meso }}(\mathrm{Pa} \mathrm{km})^{-1}\right)$, sensible heat flux $H\left(\mathrm{Wm}^{-2}\right)$, and momentum flux $\tau$ $\left(\mathrm{kgm}^{-1} s^{-2}\right)$ based on METRAS results. The red line represents the diagnosed ABL height. The flow direction is from left to right. 